\title{
Medium Access Control in Cognitive Radio Networks
}

\author{
Kaigui Bian \\ Dissertation submitted to the Faculty of the \\ Virginia Polytechnic Institute and State University \\ in partial fulfillment of the requirements for the degree of \\ Doctor of Philosophy \\ in \\ Computer Engineering \\ Jung-Min "Jerry" Park, Chair \\ Y. Thomas Hou \\ Michael S. Hsiao \\ Yaling Yang \\ James D. Arthur \\ March 30 $0^{\text {th }}, 2011$ \\ Blacksburg, Virginia
}

Keywords: Cognitive Radio, Coexistence, Medium Access Control, Control Channel, Rendezvous, Channel Hopping, Channel Assignment, Opportunistic Spectrum Sharing, Dynamic Spectrum Access.

(C) Copyright 2011, Kaigui Bian 


\title{
Medium Access Control in Cognitive Radio Networks
}

\author{
Kaigui Bian
}

\begin{abstract}
Cognitive radio $(\mathrm{CR})$ is seen as one of the enabling technologies for realizing a new regulatory spectrum management paradigm, viz. opportunistic spectrum sharing (OSS). In the OSS paradigm, unlicensed users (a.k.a. secondary users) opportunistically operate in fallow licensed spectrum on a non-interference basis to licensed users (a.k.a. incumbent or primary users). Incumbent users have absolute priority in licensed bands, and secondary users must vacate the channel where incumbent user signals are detected. A CR network is composed of secondary users equipped with CRs and it can coexist with incumbent users in licensed bands under the OSS paradigm. The coexistence between incumbent users and secondary users is referred to as incumbent coexistence, and the coexistence between CR networks of the same type is referred to as self-coexistence.

In this dissertation, we address three coexistence-related problems at the medium access control (MAC) layer in CR networks: (1) the rendezvous (control channel) establishment problem, (2) the channel assignment problem in an ad hoc CR network, and (3) the spectrum sharing problem between infrastructure-based CR networks, i.e., the 802.22 wireless regional area networks (WRANs). Existing MAC layer protocols in conventional wireless networks fail to adequately address the key issues concerning incumbent and self coexistence that emerge in CR networks. To solve the rendezvous establishment problem, we present a systematic approach, based on quorum systems, for designing channel hopping protocols that ensure a pair of CRs to "rendezvous" within an upper-bounded time over a common channel that is free of incumbent user signals. In a single radio interface, ad hoc CR network, we propose a distributed channel assignment scheme that assigns
\end{abstract}


channels at the granularity of "segments" for minimizing the channel switching overhead. By taking into account the coexistence requirements, we propose an inter-network spectrum sharing protocol that enables the sharing of vacant TV white space among coexisting WRANs. Our analytical and simulation results show that these proposed schemes can effectively address the aforementioned MAC layer coexistence problems in CR networks. 


\section{Acknowledgments}

I would like to acknowledge many people who helped me during my graduate study at Virginia Tech. First and foremost, I would like to thank my Ph.D. advisor, Dr. Jung-Min "Jerry" Park, for his generous support, excellent guidance, and rigorous scholarship. $\mathrm{He}$ not only taught me the research skills, but more importantly, inspired me to develop the independent thinking capability that will keep benefiting me in my future career. I will never forget the times and endeavors that he has investigated to make my graduate study productive.

I am so fortunate to have an excellent doctoral advisory committee, and I would like to thank Dr. Thomas Hou, Dr. Michael Hsiao, Dr. Yaling Yang, and Dr. James Arthur, for their continual support, advice, and encouragement.

During my graduate study at Virginia Tech, I have worked closely with Dr. Ruiliang Chen, Dr. Karthik Channakeshava, Dr. Janine Hiller, Dr. France Belanger, and Dr. Robert Crossler. Many of my published papers would have been impossible without their help.

The members of the ARIAS group have contributed immensely to my research work and personal life at Virginia Tech. I am especially grateful for the group members: Ruiliang Chen, Animesh Patcha, Hao Wu, Bo Gao, Behnam Bahrak, Amol Deshpande, Swati Kanaujia, Jatin Thakkar, Mike Chorzempa, Mike Snow, Maxwell Whitaker, and Daniel Ali. 
I must thank all my friends at Blacksburg, because my life here would be less wonderful without them. Dr. Ruiliang Chen, Pengyuan Yu, Dr. Jingwei Zhang, Dr. Shuquan Wang, Dr. Guodong Pang, Dr. Chao Huang, Dr. Zhongwei Li, and Dr. Ruijia Wang have offered me a lot of help starting from the first year of my life at Blacksburg. Dr. Yexin Zheng, Guanhong Pei, Shucai Xiao, Dr. Zhenhua Feng and I became good friends when they later joined the community of Virginia Tech. In the year of 2007, I found more and more lovely friends here: Min Li, Canming Jiang, Chuan Han, Hua Lin, Bin Xue, Yanzhu Ye, Shiguang Xie, Yazhe Feng, Dr. An He, Xuetao Chen, Yu Zhao, Xu Guo, Zhimin Chen, Zhenwei Cao. I would also like to thank Ting Wang, Jingyao Zhang, Ke Le, Shengzhi Shao, Yi Deng, Xinhao Ye, Guanying Wang, Zheying Guo, Huijun Xiong, Lei Sun, Yi Tang, Liguang Xie, for making the last few years of my stay at Blacksburg memorable. 


\section{Contents}

$\begin{array}{lc}\text { List of Figures } & \text { ix }\end{array}$

List of Tables $\quad$ xii

1 Introduction 1

1.1 The Rendezvous Problem in CR Networks . . . . . . . . . . . . . . 3

1.2 The Channel Assignment Problem in Ad Hoc CR Networks . . . . . . . . 6

1.3 The Spectrum Sharing Problem in Infrastructure-based CR Networks . . . 8

1.4 Organization of the Dissertation $\ldots \ldots \ldots \ldots$

2 Preliminaries $\quad 12$

2.1 Rendezvous-enabling Techniques . . . . . . . . . . . . . . . . . 12

2.1.1 Common Control Channel Based Rendezvous . . . . . . . . . . . 12

2.1.2 Channel Hopping Based Rendezvous . . . . . . . . . . . . . . 14

2.1 .3 Quorum Systems . . . . . . . . . . . . . . . . . . . 16

2.1.3.1 Cyclic quorum systems . . . . . . . . . . . . 17

2.2 Channel Assignment Strategies . . . . . . . . . . . . . . . . . . . . 18

2.2.1 Link-based Channel Assignment . . . . . . . . . . . . . . . . 18

2.2.2 Flow/component-based Channel Assignment . . . . . . . . . . 19

2.3 Self Coexistence Mechanisms in IEEE $802.22 \ldots \ldots \ldots \ldots$. . . . . . 21

2.3.1 PHY-layer Support and MAC-layer Control Messages in IEEE

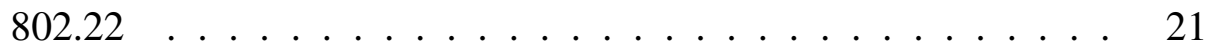

2.3.2 Non-Exclusive Spectrum Sharing: Resource Renting . . . . . . . 21

2.3.2.1 Self-interference caused by resource renting . . . . . 22

2.3.3 Exclusive Spectrum Sharing: ODSC . . . . . . . . . . . . . 23

3 Synchronous Channel Hopping Based Rendezvous 25

3.1 System Model . . . . . . . . . . . . . . . . . . . . . . . . . 25

3.2 The Quorum-based Channel Hopping System . . . . . . . . . . . 28

3.3 Metrics for Evaluating CH Systems $\ldots \ldots \ldots \ldots \ldots$ 
3.3.1 Maximum Time-to-Rendezvous . . . . . . . . . . . . . . 31

3.3 .2 Load of CH Systems . . . . . . . . . . . . . . . . . . 32

3.4 Optimal Synchronous QCH Systems . . . . . . . . . . . . . . . . . . . 34

3.4.1 Minimizing the MTTR in QCH Systems . . . . . . . . . . . . 34

3.4 .2 Minimizing the Load . . . . . . . . . . . . . . . . 36

3.5 Performance Evaluation . . . . . . . . . . . . . . . . . . . . 38

3.5.1 Impact of Time-to-Rendezvous . . . . . . . . . . . . . . . . . 40

3.5.2 Impact of Degree of Overlapping . . . . . . . . . . . 40

3.5 .3 Impact of the Load . . . . . . . . . . . . . . . . . . . 43

3.6 Summary . . . . . . . . . . . . . . . . . . . 45

4 Asynchronous Channel Hopping Based Rendezvous 47

4.1 Asynchronous Channel Hopping System . . . . . . . . . . . . . . . . 48

4.2 The ACH System with A Degree of Overlapping $m=2 \ldots \ldots \ldots$

4.2.1 Construction of AQCH Systems . . . . . . . . . . . . 53

4.3 The ACH System with Maximum Degree of Overlapping . . . . . . . . 57

4.3.1 Array-based Quorum Systems . . . . . . . . . . . . . . 57

4.3.2 Constructing ACH Systems with Maximum Degree of Overlapping 58

4.4 Optimal ACH Systems . . . . . . . . . . . . . . . . . . . . . . . 64

4.4.1 Minimum Rendezvous Probability . . . . . . . . . . . . . . 65

4.4.2 The Optimal ACH Design Problem . . . . . . . . . . . . 65

4.5 Asymmetric Optimal ACH Systems _ . . . . . . . . . . . . . . . . 67

4.6 Symmetric Optimal ACH Systems _ . . . . . . . . . . . . . . . 68

4.6.1 Limitations of Asymmetric ACH Systems . . . . . . . . . . 68

4.6.2 Construction of Symmetric ACH Systems . . . . . . . . . . 68

4.7 Comparisons of ACH Schemes . . . . . . . . . . . . . . . . . 77

4.8 Performance Evaluation . . . . . . . . . . . . . . . . . . 78

4.8.1 Simulation Setup . . . . . . . . . . . . . . . . 78

4.8.2 Impact of Degree of Overlapping . . . . . . . . . . . 80

4.8.3 Impact of Dynamic PU Traffic . . . . . . . . . . . . . . . 82

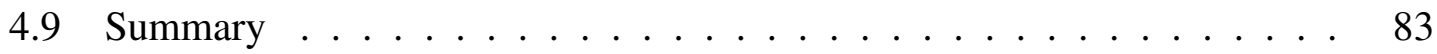

5 Segment-based Channel Assignment in Cognitive Radio Networks 85

5.1 Motivation for Segment-based Channel Assignment . . . . . . . . . . . 85

5.1.1 Segment-based Channel Assignment . . . . . . . . . . . . . . 85

5.1.2 Performance Considerations . . . . . . . . . . . . . . 88

5.1.2.1 Flow capacity without channel switching . . . . . . . 89

5.1.2.2 Flow capacity with channel switching . . . . . . . . . 92

5.2 Segment-based Channel Assignment . . . . . . . . . . . . . . . . 93

5.2 .1 Initial Handshake . . . . . . . . . . . . . . . . . . . . 93 
5.2.2 Channel Assignment . . . . . . . . . . . . . . . . . . . . . . . 94

5.2.2.1 Route request propagation . . . . . . . . . . . . . . . 94

5.2.2.2 Route reply and channel assignment . . . . . . . . . 95

5.2 .3 Segment Maintenance . . . . . . . . . . . . . . . . . . . . 98

5.2.3.1 Segment splitting . . . . . . . . . . . . . . . 98

5.2.3.2 Segment merging . . . . . . . . . . . . . . . . 99

5.3 Performance Evaluation . . . . . . . . . . . . . . . . . . . . . . . . 101

5.3.1 Simulation Setup . . . . . . . . . . . . . . . . . . . 101

5.3.2 Simulation Results . . . . . . . . . . . . . . . . . . . 101

5.3.2.1 Invariant primary user transmission . . . . . . . . 102

5.3.2.2 Segment merging in the presence of variant primary user transmission . . . . . . . . . . . 102

5.4 Summary . . . . . . . . . . . . . . . . . . . . . . . . . . 104

6 A Coexistence-aware Spectrum Sharing Protocol 105

6.1 The CASS Protocol . . . . . . . . . . . . . . . . . . . 105

6.1 .1 Basic Assumptions . . . . . . . . . . . . . . . . . . 105

6.1.2 Dynamic Switching between the Two Spectrum Sharing Modes . 106

6.1.2.1 Channel capacity in non-exclusive spectrum sharing mode 107

6.1.2.2 Channel capacity in exclusive spectrum sharing mode . 107

6.1.2.3 An approach of switching between two modes . . . . . 109

6.1.3 The Channel Selection Mechanism _. . . . . . . . . . . . 110

6.1.4 The Channel Contention Procedure . . . . . . . . . . . . . . 112

6.1.4.1 Contention priority number . . . . . . . . . 112

6.1.4.2 Token assignment . . . . . . . . . . . . . . . . 112

6.1.4.3 Contention resolution rule . . . . . . . . . . . . 113

6.1.4.4 Weighted fairness . . . . . . . . . . . . . . . 114

6.2 Performance Evaluation . . . . . . . . . . . . . . . . . . . 115

6.2 .1 The 3 -BS Scenario . . . . . . . . . . . . . . . . 116

6.2 .2 The 9 -BS Scenario . . . . . . . . . . . . . . . . . . 118

6.3 Related Work . . . . . . . . . . . . . . . . . . . . . . 120

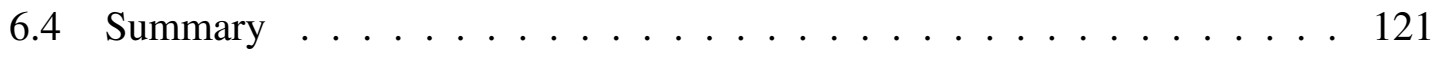

7 Conclusions and Future Work 122

7.1 Conclusions . . . . . . . . . . . . . . . . . . . 122

7.2 Future Work . . . . . . . . . . . . . . . . . . 123

$\begin{array}{ll}\text { Bibliography } & 127\end{array}$

$\begin{array}{ll}\text { A Authors' Publications } & 133\end{array}$ 


\section{List of Figures}

1.1 Taxonomy of coexistence in wireless networks. In this dissertation, we focus on problems that are related to incumbent and self coexistence in CR networks. . . . . . . . . . . . . . . . . . 3

2.1 Distributed channel negotiation process between a sender and a receiver. . 14

2.2 A taxonomy of channel assignment strategies. . . . . . . . . . . 20

2.3 Inter-cell Resource Renting in 802.22. . . . . . . . . . . . . . 23

3.1 An illustration of QCH system $Q$ with $m=3$ and $k=3$. Any two sequences overlap on three channels. We use the quorum system $S=$ $\{\{0,1\},\{0,2\},\{1,2\}\}$ over $U=\{0,1,2\}$ to construct $Q$. The numbers inside the slots denote channel index values: bold-font values denote channel indexes from $R$ and the non-bold font values denote channel indexes randomly chosen from $\{0, \ldots, N-1\} \ldots \ldots \ldots \ldots$

3.2 An L-QCH system, $Q$, with $m=N$ rendezvous channels and $\tau=7$. To construct $Q$, we use a minimal cyclic quorum system $S=\{\{0,1,3\},\{1,2,4\}$, $\{2,3,5\},\{3,4,6\},\{4,5,0\},\{5,6,1\},\{6,0,2\}\}$ under $U=\mathbf{Z}_{7}$. The channel indexes for "blank" slots are randomly selected from $[0, N-1]$. Any two sequences in this system overlap on all $N$ channels. . . . . . . . . 36

3.3 Effect of time-to-rendezvous and channel switching. . . . . . . . . . 41

3.4 Time-to-rendezvous with incumbent traffic. . . . . . . . . . . . 42

3.5 Throughput of non-disjoint flows with incumbent traffic. . . . . . . . 43

3.6 Effect of load on throughput. . . . . . . . . . . . . . . 44

4.1 An ACH system $Q$ with $m=2$ and $T=k=9$. The universal set, $U$, is $\mathbf{Z}_{9}, S$ is constructed using $D=\{0,1,2,4\}$, and $S^{\prime}$ is constructed using $D^{\prime}=\{3,5,6,7,8\}$. Note that $D^{\prime} \cap D=\emptyset$. The numbers inside the slots represent the channel index values. . . . . . . . . . . . . 
4.2 Two quorums, $p$ and $q$, each with 8 elements, in an array-based quorum system. Both quorums are constructed using a $3 \times 6$ array. An array-based quorum includes a column of elements (squares colored in grey) and a span of elements (squares labeled with a ' $S$ '). A span and a column have one element in common which is not counted twice. One quorum's column must have an intersection with a span of another. For instance, in the figure, $p$ 's column intersects $q$ 's span at the square that is located in the first row of the third column. Similarly, $p$ 's span intersects $q$ 's column at the square located in the first row of the sixth column. Note that performing cyclic rotation by one slot to either of the array-based quorums yields a third array-based quorum (e.g., $q^{\prime}=\operatorname{rotate}(q, 1)$ ), and the intersection property is maintained after the cyclic rotation. The elements in common between $p$ and $q^{\prime}$ are marked with circle markings, and those between $q$ and $q^{\prime}$ are marked with square markings. . . . . . . . . . . . .

4.3 An ACH system $H$ when $N=3, r=3, l=5$, and the receiver's clock is ahead of the sender's clock by $(2+\delta)$ timeslots where $\delta \leq 1 / 2$. The sequence arrays of the sender and the receiver that generated the respective sequences are also shown. It can be easily seen that the two $\mathrm{CH}$ sequences have $N$ distinct rendezvous channels despite the clock drift and that the overlap duration on each rendezvous channel is $(1-\delta) \ldots . . . . .$. .

4.4 An asymmetric design of an optimal $\mathrm{ACH}$ system when $N=3$. The sender's clock is ahead of the receiver's clock by $(2+\delta)$ timeslots where $\delta \leq 1 / 2$. The sequence arrays of the sender and the receiver that generated the respective $\mathrm{CH}$ sequences are shown. It can be easily seen that the two $\mathrm{CH}$ sequences have $N$ distinct rendezvous channels despite the clock drift and that the overlap duration in each rendezvous channel is $(1-\delta) . .$.

4.5 Illustration of the three cases Lemma 1's proof . . . . . . . . . . . . . . .

4.6 Construction of a $\mathrm{CH}$ sequence for the symmetric $\mathrm{ACH}$ scheme. In the above figure, node $x$ 's ID sequence is $\alpha=\{1,0\}, n=2, z=\{0,0\}$ and $o=\{1,1\}$. Node $x$ 's column- and span-based sequences are $u^{x}$ and $v^{x}$, respectively. . . . . . . . . . . . . . . . . 
4.7 Motivation for using two concatenated column/span-based sequences instead of a single sequence. Suppose node $x$ generates column and spanbased sequences $u^{x}$ and $v^{x}$; similarly, node $y$ generates sequences $u^{y}$ and $v^{y}$. Using two concatenated column or span-based sequences in the $\mathrm{CH}$ sequence construction method (method 2 in the figure), the maximum rendezvous diversity is guaranteed when two channel hopping nodes have a clock drift that is less than a frame length. In the example, given $N$ channels and a $\mathrm{CH}$ period of $T$, rotate $\left(u^{x}, T-k\right)$ yields another column-based sequence for node $x$, which overlaps with node $y$ ' span-based sequence $v^{y}$ over $N$ channels. On the contrary, the method of using a single column or span-based sequence (method 1 in the figure) is unable to guarantee such maximum rendezvous diversity, e.g., $u^{x}$ may not have $N$ rendezvous channels with a sequence concatenated by parts of $v^{y}$ and $u^{y}$. . . . . . . 74

4.8 The proportion of rendezvous pairs vs. number of primary transmitters. $\quad$ - $\quad 80$

4.9 Average TTR vs. number of primary transmitters. . . . . . . . . . . . 81

4.10 Rendezvous rate. . . . . . . . . . . . . . . . . . . 82

4.11 Average TTR vs. mean of PU's idle period when $b=10 \ldots \ldots 3$

4.12 Average TTR vs. mean of PU's idle period when $b=1 \ldots . . . . . . .84$

5.1 An illustration of segment-based channel assignment. . . . . . . . . . . . . . 88

5.2 Channel and slot assignments for contending flows. . . . . . . . . . . 89

5.3 Channel and slot assignments for intersecting flows. . . . . . . . . . . . 91

5.4 Two segments are formed via the segment-based channel assignment approach. . . . . . . . . . . . . . . . . . 98

5.5 Two segments merge into one if primary user 2 is silent. . . . . . . . . 100

5.6 Average throughput vs. number of channels. . . . . . . . . . . . . . 102

5.7 Comparisons on the average throughputs of three protocols. . . . . . . . . 103

6.1 Incumbent idle time period and channel contention periods. . . . . . . . 108

6.2 Throughput of BS $b$ vs. distance $d$ in the 3-BS scenario. . . . . . . . . . 117

6.3 Throughput vs. Number of active incumbent transmitters in the 9-BS scenario. . . . . . . . . . . . . . . . . . 118

6.4 Throughput vs. Incumbent transmission rate in the 9-BS scenario. . . . . 119

6.5 Throughput vs. Channel switch delay in the 9-BS scenario. . . . . . . . . 120 


\section{List of Tables}

3.1 A comparison of synchronous $\mathrm{CH}$ schemes. . . . . . . . . . 38

4.1 A comparison of asynchronous $\mathrm{CH}$ schemes. . . . . . . . . . . 78

6.1 Default simulation parameter values. . . . . . . . . . . . 116 


\section{Chapter 1}

\section{Introduction}

The proliferation of wireless applications operating in unlicensed spectrum bands has resulted in the overcrowding of those bands. In contrast, recent studies have shown that most licensed bands are under-utilized [21]. To address the spectrum shortage problem, the Federal Communications Commission (FCC) is considering the adoption of a new regulatory spectrum management paradigm in which licensed bands are opened up to unlicensed operations [22]. This new regulatory model is often called "opportunistic spectrum sharing (OSS)". In the OSS paradigm, unlicensed users (a.k.a. secondary users) opportunistically access licensed spectrum on a non-interference basis to licensed users (a.k.a. incumbent or primary users).

The cognitive radio (CR) is seen as the key enabling technology for realizing OSS. A cognitive radio has the capability to sense its environment and adapt its mode of operation to achieve its performance objectives [1,27]. In CR networks, spectrum opportunities ("white spaces") in licensed bands are identified by the process of spectrum sensing [14]. 
Once spectrum opportunities are identified, those spectrum resources are mapped into logical channels, where each logical channel is the unit of channel assignment. A CR network composed of secondary users (nodes ${ }^{1}$ ) equipped with CRs can coexist with incumbent users in licensed bands under the OSS paradigm.

The coexistence of wireless networks can be broadly classified into two categories: vertical coexistence and horizontal coexistence.

- Vertical coexistence refers to the coexistence of two or more networks that have different priorities to access spectrum. For instance, in CR networks, incumbent users have priority over secondary users when accessing the licensed spectrum bands.

- Horizontal coexistence refers to the coexistence of two or more unlicensed networks that have equal priority to access spectrum.

In the OSS paradigm, the coexistence between incumbent users and secondary users is referred to as incumbent coexistence. There exists a significant body of work on incumbent coexistence $[10,12,13,24,52,66]$, and it has been attracting significant interest from academia and industry. In contrast, horizontal coexistence has garnered less attention thus far. Horizontal coexistence can be further categorized into:

- Heterogeneous coexistence that refers to the coexistence of networks that employ different wireless technologies (e.g., the coexistence between WiFi and Bluetooth [29, 72], the coexistence of heterogeneous wireless networks over TV white space [30]);

- Homogeneous coexistence (a.k.a. self coexistence) that refers to the coexistence of networks that employ the same wireless technology (e.g., neighboring CR networks

\footnotetext{
${ }^{1}$ We use "node" and "secondary user" interchangeably.
} 


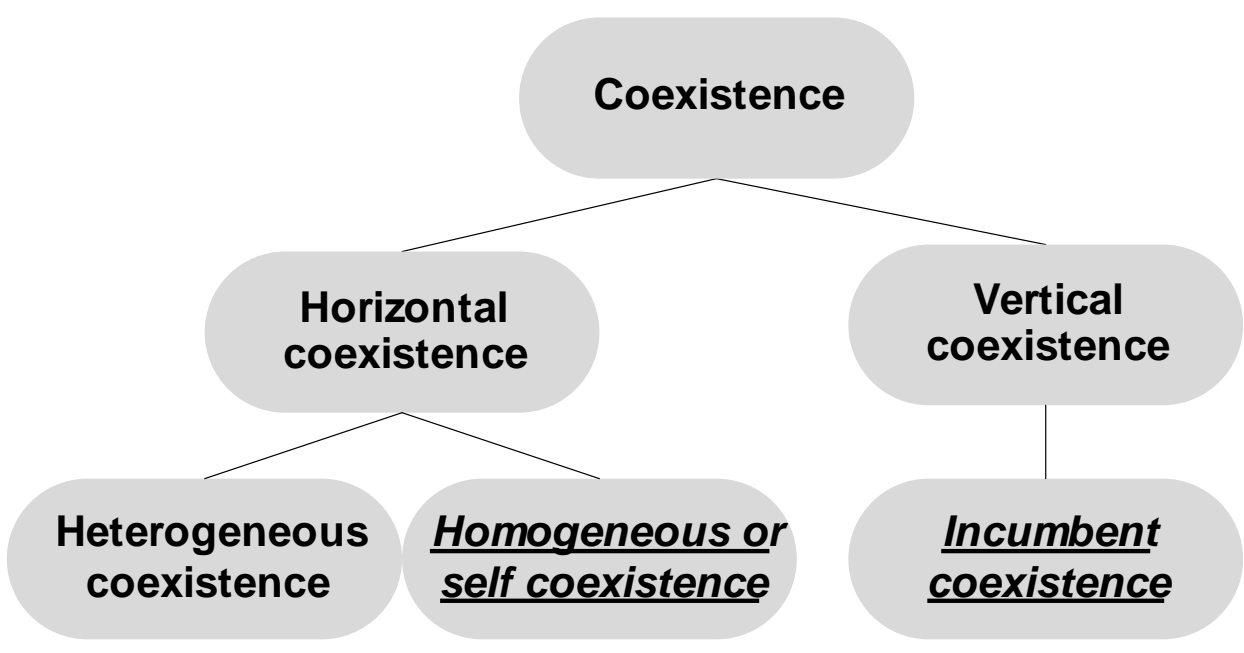

Figure 1.1: Taxonomy of coexistence in wireless networks. In this dissertation, we focus on problems that are related to incumbent and self coexistence in CR networks.

of the same type [8,41], or neighboring 802.11 hotspots [43]).

Figure 1.1 illustrates the taxonomy of coexistence problems in wireless networks.

In this dissertation, we address three medium access control (MAC) layer problems that are related to incumbent and self coexistence in CR networks: (1) the rendezvous (control channel) establishment problem, (2) the channel assignment problem in an ad hoc CR network, and (3) the spectrum sharing problem between infrastructure-based CR networks, i.e., the 802.22 wireless regional area networks (WRANs). Existing MAC layer protocols in conventional wireless networks fail to adequately address the key issues concerning incumbent and self coexistence that emerge in CR networks.

\subsection{The Rendezvous Problem in CR Networks}

Rendezvous or control channel establishment in CR networks requires two communicating nodes to "rendezvous"-i.e., find each other on a rendezvous channel—prior to initiating 
data communications. Existing rendezvous-enabling techniques can be classified into two approaches: common control channel-based rendezvous [15, 34, 45, 55, 69] and channel hopping $(\mathrm{CH})$-based rendezvous (a.k.a. parallel rendezvous) $[4,56]$. Recall that secondary nodes are required to refrain from transmitting in the channels where primary user signals are detected, and the availability of the channels changes dynamically due to the appearance and disappearance of primary user signals.

- The use of a single common control channel simplifies the rendezvous process but may create a network performance bottleneck and it acts as a single point of failure $[4,44]$.

- Channel hopping-based rendezvous avoids the drawbacks of the common control channel and creates parallel rendezvous-multiple pairs of nodes are able to rendezvous on different channels simultaneously.

To ensure successful rendezvous (of secondary node pairs that need to establish a control channel) with a high probability, the $\mathrm{CH}$-based rendezvous process needs to support rendezvous in multiple channels so that the risk of rendezvous failure due to the appearance of primary user signals is minimized. Ideally, the rendezvous channels should be spread out over all available channels to maximize the chance of rendezvous success. We coin the term rendezvous problem in the context of time-synchronous or time-asynchronous CR networks to denote the following problem: How can two channel hopping secondary nodes, with or without clock synchronization, achieve pairwise rendezvous with a guaranteed latency upperbound in the presence of primary user transmissions? 
Most existing $\mathrm{CH}$ protocols (e.g., $[4,56])$ assumes the clock synchronization, which implies that the communicating nodes are able to align the boundaries of their $\mathrm{CH}$ sequences. Meanwhile, it is worth noting that there are $\mathrm{CH}$ schemes that enable rendezvous without requiring clock synchronization-e.g., the sequence-based rendezvous protocol [19]. However, this protocol only supports a very limited number of pairwise rendezvous channels when two nodes' clocks are asynchronous, which increases the chances of rendezvous failure and, in turn, link establishment failure. A $\mathrm{CH}$ protocol needs to satisfy two critical requirements: (1) establish pairwise rendezvous between two $\mathrm{CH}$ sequences of the sender and the receiver on multiple channels or every available channel in the ideal case; and (2) ensure that the two nodes' $\mathrm{CH}$ sequences achieve rendezvous with an upper bounded time-to-rendezvous (TTR), even if their clocks are asynchronous. The first requirement ensures that the rendezvous failures due to the presence of primary user signals are reduced or minimized. The second requirement makes it possible to upper bound the medium access latency of secondary user nodes since the time required for rendezvous is often unpredictable when establishing links in distributed networks.

Most, if not all, of the existing work on $\mathrm{CH}$ schemes $[4,19,54,56,62]$ only provide ad-hoc approaches for generating $\mathrm{CH}$ sequences and evaluating their properties. In a timesynchronous CR network, we present a systematic approach in this dissertation, based on quorum systems, for designing and analyzing $\mathrm{CH}$ protocols for the purpose of control channel establishment. The proposed approach, called Quorum-based Channel Hopping $(Q C H)$ system, utilizes the intersection property of quorum systems to generate $\mathrm{CH}$ sequences that enable rendezvous on multiple channels between any two $\mathrm{CH}$ sequences. Under the assumption of global clock synchronization, we describe two QCH systems. The 
first system minimizes the upperbound of the TTR value between any two $\mathrm{CH}$ sequences. The second system evenly distributes the rendezvous points over different timeslots during a $\mathrm{CH}$ period, thereby alleviating the rendezvous convergence problem. To address the rendezvous problem in time-asynchronous CR networks, we propose three asynchronous channel hopping $(\mathrm{ACH})$ systems that do not require global clock synchronization. The first $\mathrm{ACH}$ system is constructed using the cyclic quorum system, the second $\mathrm{ACH}$ system is constructed using the array-based quorum system, and the third ACH system is built by leveraging bit-sequence design techniques. Note that, $\mathrm{CH}$ sequences in an $\mathrm{ACH}$ system are generated by exploiting the rotation closure property of quorum systems, which enables

the TTR between any two nodes' $\mathrm{CH}$ sequences to be upper bounded without requiring clock synchronization.

\subsection{The Channel Assignment Problem in Ad Hoc CR Net- works}

In the past few years, several channel assignment strategies have been proposed for conventional multichannel wireless networks. In link-based channel assignment, channel assignment is performed at the granularity of a link between two given nodes. All packets on this link are transmitted on the same channel as long as the channel assignment does not change. The flow-based approach assigns channels at the granularity of a flow, i.e., packets of a flow are scheduled on the same channel. In [64], Vedantham et al. proposed component-based channel assignment. (See [64] for a precise definition of a component.) In this approach, nodes belonging to a set of intersecting flows (defined as a "component") 
are assigned to the same channel. Vedantham et al. showed that the component-based approach's theoretical performance does not lag significantly behind finer granularity channel assignment (i.e., link- and flow-based) approaches when channel switching delay is ignored. It is not possible to apply component-based/flow-based channel assignment to CR networks because not all of the secondary nodes within a given component/flow have access to the same set of channels due to the temporal and spatial spectrum variability caused by primary user's spectrum utilization. Hence, it is no surprise that existing channel assignment schemes for CR networks use the link-based approach $[9,11,69,70]$. Unfortunately, if channel switching delay is significant, it is also showed in [64] that the component-based channel assignment strategy has practical advantages over existing strategies, such as the avoidance of channel switching delay and overhead. The link-based approach suffers from these practical limitations because a single radio node serving two links assigned with different channels has to switch between channels when forwarding data packets, and thus degrading the overall network performance.

In this dissertation, we consider the channel assignment problem in a single radio interface, ad hoc CR network, where each secondary user is equipped with a half-duplex cognitive radio. We propose a channel assignment strategy that uses the "segment" as the granularity of channel assignments. Nodes within intersecting flows (i.e., a component) can be grouped into one or more sets according to the set of channels available to each node-i.e., all nodes in the same segment have access to the same set of common channels. The channel assignment problem has an added dimension of complexity in the context of CR networks that does not exist in the context of conventional wireless networks. This complexity is the need to switch channels due to the temporal and spatial 
spectrum variability caused by incumbent users' spectrum utilization. The proposed segment based channel assignment strategy deals with the spectrum variability problem by incorporating an adaptive segment maintenance mechanism that includes mechanisms for segment merging and segment splitting. During the lifetime of a flow, a given segment may need to split into smaller segments or merge as part of a larger segment due to the appearance/disappearance of primary user signals in the current operating band. The splitting/merging of segments leads to channel switching. Channel switching is one of the major factors leading to performance degradation in link based schemes. Practical limitations caused by channel switching include switching delay, scheduling overhead, and synchronization requirements. Although channel switching cannot be avoided completely in the context of CR networks, the proposed channel assignment scheme minimizes the need for it by using the segment as the granularity of channel assignment.

\subsection{The Spectrum Sharing Problem in Infrastructure-based CR Networks}

IEEE 802.22 specifies the air interface for a Wireless Regional Area Network (WRAN) that operates in fallow TV broadcast bands [31]. An 802.22 cell is a single-hop, pointto-multipoint wireless network composed of a Base Station (BS) and several Consumer Premise Equipments (CPEs). Incumbent services refer to TV broadcasting services or services for Part 74 devices $^{2}$ (wireless microphones) operating in TV bands, and secondary users refer to IEEE 802.22 entities (BS and CPEs). There are two main objectives in

\footnotetext{
${ }^{2}$ Part 74 devices are low-power wireless devices, such as wireless microphones, which are licensed to operate in the TV broadcast bands.
} 
self-coexistence between 802.22 WRANs: minimizing the self interference between cochannel overlapping cells and satisfying the QoS requirements of the cells' admitted service workloads in an OSS environment.

802.22 prescribes two types of inter-BS dynamic resource sharing mechanisms so that cells can satisfy the QoS of their admitted workloads. A BS in need of spectrum (renter BS) is allowed to selectively rent candidate channels of neighboring cells (surplus channels that can be given up) via the Resource Renting mechanism. Note that a candidate channel of one cell may be an active channel (a channel in use) of another cell. Thus, Resource Renting is a non-exclusive spectrum sharing scheme that enables neighboring BSs to share one or more channels. 802.22 also defines an exclusive spectrum sharing scheme called inter-BS On-Demand Spectrum Contention (ODSC). In ODSC, a BS in need of spectrum (contention source) selectively contends for candidate channels of neighboring BSs (contention destinations). If the contention source wins the contention, it occupies the contended channels exclusively, while the contention destinations vacate those channels via channel switching.

Unfortunately, 802.22's inter-BS resource sharing mechanisms do not consider incumbent coexistence issues when a renter BS selects channels to rent. If the appearance of incumbent signals in the rented channels is frequent, it is likely to have two negative impacts: the QoS degradation due to frequent channel switches in the renter cell when incumbent signals are detected by the renter and interference experienced by incumbent users. Another shortcoming of the resource sharing mechanisms is their failure to adequately address self-coexistence issues. The non-exclusive spectrum sharing scheme does little to prevent self-interference among co-channel overlapping cells, which can render 
802.22 networks to be useless [18]. Although the exclusive spectrum sharing scheme can avoid self-interference altogether, it incurs heavy control overhead due to its channel contention procedure. Thus, an appropriate tradeoff has to be made between self-interference and control overhead. An ideal spectrum sharing mechanism needs to have the ability to dynamically "switch" between non-exclusive and exclusive spectrum sharing when the situation requires it.

In this dissertation, we propose an inter-BS Coexistence-Aware Spectrum Sharing $(C A S S)$ protocol. CASS has the following noteworthy features: (1) it supports both nonexclusive and exclusive spectrum sharing and can dynamically switch between the two to minimize self-interference while keeping control overhead (induced by channel contentions) under control; (2) it uses a new channel selection algorithm that utilizes spectrum sensing results in order to minimize the likelihood of interference to incumbent transmissions; and (3) it uses an inter-BS channel contention procedure that enables a BS in need of more channels to borrow channels from its neighboring cells more readily (compared to 802.22 's ODSC).

Our analytical and simulation results show that these proposed schemes can effectively address the aforementioned MAC layer coexistence problems in CR networks.

\subsection{Organization of the Dissertation}

The rest of this dissertation is organized as follows. In Chapter 2, we introduce background knowledge and related work. The synchronous channel hopping based rendezvous protocol is presented in Chapter 3, followed by the asynchronous channel hopping based rendezvous protocol in Chapter 4. In Chapter 5, we provide the segment-based channel 
assignment algorithm for single radio interface, CR networks. In Chapter 6, the CASS protocol is elaborated for improving the coexistence of 802.22 WRANs. Finally, we conclude this research and outline the future work in Chapter 7. 


\section{Chapter 2}

\section{Preliminaries}

In this chapter, we provide background knowledge on the rendezvous problem, the channel assignment problem and the spectrum sharing problem that will facilitate the understanding of the various nomenclature and concepts that will be used throughout the dissertation.

\subsection{Rendezvous-enabling Techniques}

\subsubsection{Common Control Channel Based Rendezvous}

In a typical multi-channel MAC protocol, nodes contend for channels by exchanging MAC layer control packets in the common control channel (CCC) [55,67]. If there is no access point or base station, channel negotiation is carried out in a distributed manner, between each pair of sender and the intended receiver. We describe CCC-based rendezvous techniques using the example shown in Figure 2.1. Since there is no existing standard that defines such a MAC layer, the discussions given here are based on the common features 
shared by most of the MAC protocols using a global or local common control channel $[15,34,45,55,69]$. During channel negotiation phase, the following types of MAC frames are utilized: Free Channel List (FCL), SELection (SEL), and REServation (RES). Figure 2.1 shows a simple example of a channel negotiation process between a sender and a receiver. Here, the "sender" is the host that transmits the MAC data frames, and the "receiver" is the host that receives the MAC data frames. The sender first identifies fallow spectrum bands and maps them into logical channels. Then it obtains a free channel list (FCL frame) and sends this to the receiver after waiting a random back-off time. Upon receiving the FCL, the receiver identifies available data channels common to both sides, and then selects one data channel according to a data channel selection policy. The channel selection is indicated in a SEL frame and sent back to the sender. After receiving the SEL frame, the sender notifies its neighbors of the channel selection via a channel reservation message (RES frame). Neighbor nodes refrain from transmitting by maintaining a network allocation vector (NAV) specified in the FCL and SEL frames overheard during the channel negotiation process. Using the above process, a sender and a receiver select a data channel for communicating.

A common control channel will cause two problems in multi-channel wireless networks. First, relying on a common control channel may create a bottleneck (a la control channel saturation problem) for the network performance [4] [55]. The second problem that may arise from the use of a common control channel is the single point of failure problem when the control channel is targeted by jamming attacks. In [40], channel hopping is used for recovering the control channel for a cluster of ad hoc nodes whenever the common control channel is jammed. The hopping sequence of the control channel 


\section{Common Control Channel}

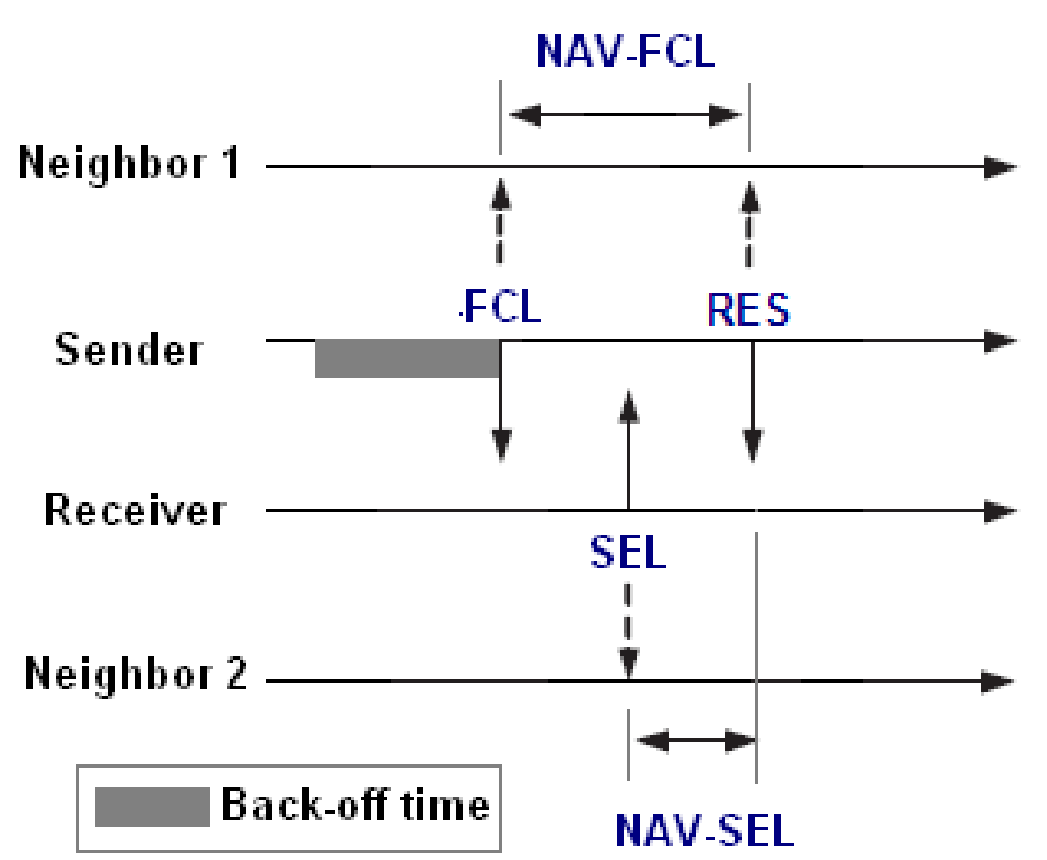

Figure 2.1: Distributed channel negotiation process between a sender and a receiver.

is pseudo random, but it is pre-determined. The hopping sequence is distributed by the cluster head to all nodes in the same cluster. In [17], a dynamic common control channel scheme is proposed, in which the common control channel can switch to other channels if the current spectrum band is occupied by the incumbent users. However, control channel hopping techniques cannot solve the control channel saturation problem.

\subsubsection{Channel Hopping Based Rendezvous}

In the design of multi-channel MAC protocols, the use of channel hopping (or parallel rendezvous) techniques have been proposed to enable rendezvous between a pair of communicating nodes, and the $\mathrm{CH}$ sequences are carefully generated to guarantee that different 
$\mathrm{CH}$ sequences must have overlap.

Blind rendezvous $(B R)$ channel hopping [54]. In this scheme, each node hops from one channel to another randomly. At a particular instant, a node occupies one of these channels with probability $1 / N$, where $N$ is the total number of channels. When two nodes occupy the same channel at the same time, rendezvous occurs. The BR scheme does not guarantee a bounded TTR between any two sequences.

Slotted Seeded Channel Hopping (SSCH) [4]. Each node is allowed to have one or multiple (channel, seed)-pairs to determine its $\mathrm{CH}$ sequences. $\mathrm{SSCH}$ allows $(N-1)$ seeds, where $N$ is the total number of channels. Each sequence period includes a parity slot at which time instant all nodes with the same seed are guaranteed to rendezvous on a channel indicated by the seed value. When each node selects one (channel, seed)-pair, the resulting sequence period is $(N+1)$ timeslots, and each pair of sequences rendezvous exactly once within a period. By design, $\mathrm{SSCH}$ is a synchronous $\mathrm{CH}$ system (the boundaries of nodes' $\mathrm{CH}$ sequences should be aligned), although results in [4] show that it can tolerate moderate clock skew. The amount of clock skew used in [4] to evaluate SSCH is very small relative to one slot duration.

Sequence-based rendezvous (SeqR or SR) [19]. Every node using SR follows the same pre-determined sequence. The common sequence, $u^{*}$, is built by first selecting a permutation of elements in $\mathbf{Z}_{N}$, where $N$ is the total number of channels and $i \in[0, N-1]$ is a channel index. Then, the elements of the permutation is interspersed with $N$ identical permutations. For example, when $N=3$, one can select a permutation such as $\{0,2,1\}$, and the resulting $\mathrm{CH}$ sequence $u^{*}$ would be an ordered sequence of channel indexes, such 
as

$$
\{0,0,2,1,2,0,2,1,1,0,2,1\} \text {. }
$$

The elements in the permutation $\{0,2,1\}$ is interspersed with the three replications of the same permutation. The sequence period is $N(N+1)$ timeslots.

\subsubsection{Quorum Systems}

In this dissertation, the quorum system will be used for designing $\mathrm{CH}$ sequences in a systematic way.

Definition 1. Given a finite universal set $U=\{0, \ldots, n-1\}$ of $n$ elements, a quorum system $S$ under $U$ is a collection of non-empty subsets of $U$, which satisfies the intersection property:

$$
p \cap q \neq \emptyset, \forall p, q \in S
$$

Each $p \in S$ (which is a subset of $U$ ) is called a quorum.

The quorum systems have been used for designing the wake-up schedule in wireless sensor or ad hoc networks for power saving $[36,61,71]$. The intersection property of quorum systems is exploited to create the overlap between wake-up schedules of two sensor nodes so that they could discover each other without keeping awake all the time. As a result, the power consumption is reduced. In this dissertation, we use the intersection property of quorum systems to create overlap between $\mathrm{CH}$ sequences when their boundaries are synchronized. When the clock synchronization is unavailable, a second property of quorum systems - rotation closure property — will be used to maintain the overlap between asynchronous $\mathrm{CH}$ sequences. 


\subsubsection{Cyclic quorum systems}

Here, we provide some definitions related to cyclic quorum systems since those systems are utilized to design channel hopping schemes in Chapters 3 and 4. The cyclic quorum system, first introduced in [42], can be constructed using cyclic difference sets in combinatorial theory [57].

Definition 2. A set $D=\left\{a_{1}, a_{2}, \ldots, a_{\kappa}\right\} \subset \mathbf{Z}_{n}$ is called a relaxed cyclic $(n, \kappa)$-difference set iffor every $d \not \equiv 0(\bmod n)$ there exists at least one ordered pair $\left(a_{i}, a_{j}\right)$, where $a_{i}, a_{j} \in$ $D$, such that $a_{i}-a_{j} \equiv d(\bmod n)$. Here, $\mathbf{Z}_{n}$ denotes the set of nonnegative integers less than $n$.

Definition 3. A group of sets

$$
B_{i}=\left\{a_{1}+i, a_{2}+i, \ldots, a_{\kappa}+i\right\} \quad \bmod n, i \in\{0,1, \ldots, n-1\}
$$

is a cyclic quorum system if and only if $D=\left\{a_{1}, a_{2}, \ldots, a_{\kappa}\right\}$ is a relaxed cyclic $(n, \kappa)$ difference set.

For example, $D=\{0,1,3\}$ is a relaxed cyclic $(7,3)$-difference set under $\mathbf{Z}_{7}$ since each $d \in\{1, . ., 6\}$ is congruent to the difference of two elements in $D$. Given $D$,

$$
S=\left\{B_{0}, B_{1}, \ldots, B_{6}\right\}
$$

is a cyclic quorum system under $\mathbf{Z}_{7}$, where

$$
B_{i}=\{0+i, 1+i, 3+i\} \quad \bmod 7, i=\{0,1, \ldots, 6\}
$$

It was proven in [36] that any quorum $q$ in a cyclic quorum system under $U=\{0, \ldots, n-1\}$ must have a cardinality $|q| \geq \sqrt{n}$. 
Given any $n$, a difference set as small as $\kappa \approx \sqrt{n}$ can be found when $\kappa^{2}-\kappa+1=n$ and $\kappa-1$ is a prime power. Such a difference set is called the Singer difference set [16], which is the minimal difference set whose size $\kappa$ approximates the lower bound $\sqrt{n}$. Hence, cyclic quorum systems defined by the Singer difference sets are minimal cyclic quorum systems in the sense that their quorum sizes are close to the theoretical lower bound. For example, the set $\{1,2,4\}$ under $\mathbf{Z}_{7}$ is a Singer difference set when $\kappa=3$.

Any set $D$ that contains $\left\lceil\frac{n+1}{2}\right\rceil$ elements of $\mathbf{Z}_{n}$ is a relaxed cyclic $\left(n,\left\lceil\frac{n+1}{2}\right\rceil\right)$-difference set and a cyclic quorum system $S=\left\{B_{0}, B_{1}, \ldots, B_{n-1}\right\}$ can be constructed based on $D$ according to Definition 3. Since $D$ contains more than half of the elements in $\mathbf{Z}_{n}$, we refer to such a cyclic quorum system, $S$, as a majority cyclic quorum system. For example,

$$
S=\{\{0,1,2\},\{1,2,3\},\{2,3,0\},\{3,0,1\}\}
$$

is a majority cyclic quorum system under $\mathbf{Z}_{4}$.

\subsection{Channel Assignment Strategies}

A taxonomy of channel assignment strategies is shown in Figure 2.2. Not that, to avoid the interference to incumbent users in CR networks, we derive the segment-based channel assignment strategy from the component-based approach.

\subsubsection{Link-based Channel Assignment}

All packets on a wireless link between two nodes are transmitted on the same channel until the channel assignment decision expires. Each link in a flow can choose any one of the 
free channels. Several approaches such as common control channel based channel negotiation [55,67], D1EC [3], SSCH [4] and MMAC [55] fall under the category of link level assignment. Note that existing channel assignment strategies for CR networks take the link-based approach, such as $[9,11,69,70]$. The major pitfall of a link-based approach is the significant channel switching delay incurred when a node serves two links on different channels. Therefore, they suffer from the practical limitations of the link-based strategy. Those limitations include switching delay, synchronization requirements, and scheduling overhead. Note that channel switching is required in the link-based approach when an intersection node serves two links in different channels.

\subsubsection{Flow/component-based Channel Assignment}

In flow-based channel assignment, all packets belonging to a flow are transmitted on the same channel. Different flows may operate on different channels. A graph is connected if there is a path connecting every pair of vertices. A graph that is not connected can be divided into connected components (disjoint connected subgraphs). A component in the context of channel assignment is similarly defined as a connected subgraph in the network flow graph, which is composed of nodes belonging to intersecting flows. In componentbased channel assignment, all nodes within a component are assigned the same channel. If there are no intersecting flows, the component-based assignment is equivalent to the flow-based assignment. 


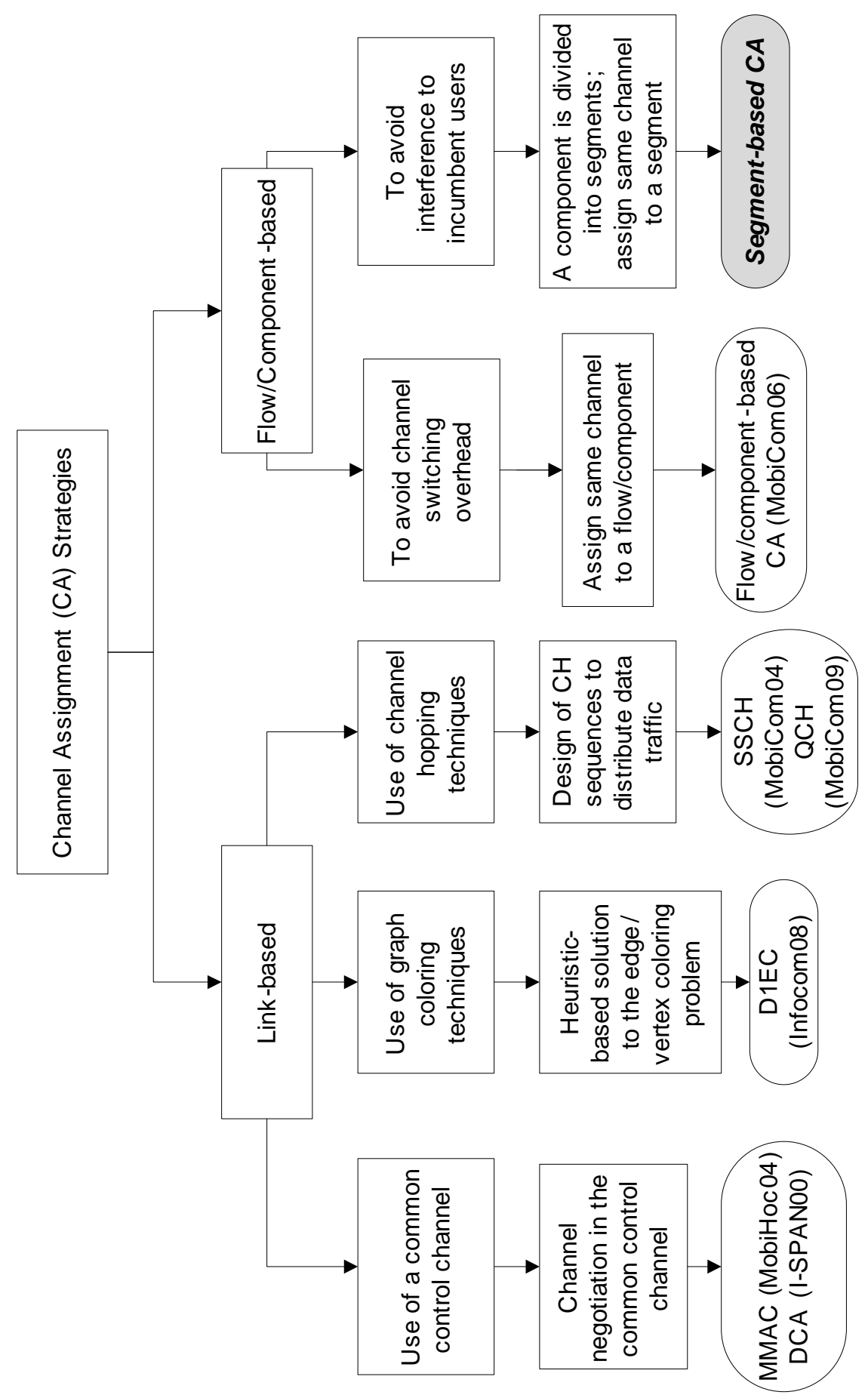

Figure 2.2: A taxonomy of channel assignment strategies. 


\subsection{Self Coexistence Mechanisms in IEEE 802.22}

\subsubsection{PHY-layer Support and MAC-layer Control Messages in IEEE 802.22}

802.22 employs a Distributed Spectrum Sensing (DSS) framework to identify fallow licensed bands that are free of incumbent signals. In a cell, every CPE is required to report its local spectrum sensing results to its BS via intra-cell measurement (control) messages. The BS has multiple radio interfaces for monitoring a number of channels simultaneously, and it determines the existence of incumbent signals on those channels using the local spectrum sensing reports from CPEs.

802.22 also defines inter-cell control messages as beacons to support the inter-BS communication. In every 802.22 time frame, there is a self-coexistence window for a BS to exchange beacons with neighboring BSs on multiple channels. If a beacon from a neighboring $\mathrm{BS}$ is received on a channel, that neighboring $\mathrm{BS}$ is deemed as a co-channel BS by the receiver BS. When direct inter-BS communication is infeasible, a BS instructs its associated CPEs (a.k.a. bridge CPEs) to listen for beacons from overlapping cells and report the information back. This feature enables a BS to obtain accurate information about other cells.

\subsubsection{Non-Exclusive Spectrum Sharing: Resource Renting}

In an inter-BS dynamic resource sharing process, 802.22 defines offerers to be the BSs that have available resources and the renter to be the BS in need of spectrum. A renter BS 
initiates a Resource Renting process by broadcasting a resource request using inter-cell beacons. Upon reception of the resource request, neighboring BSs respond by broadcasting beacons that indicate their active channel sets and candidate channel sets. The union of candidate channel sets from neighboring BSs forms the renter BS's grand candidate channel set from which it selects channels to rent. The renter BS sends beacons to the offerers indicating the selected channel number and the duration of renting time. The Resource Renting process is concluded when the offerers transmit an acknowledgement back to the renter. 802.22 prescribes the following rule that the renter needs to comply with when selecting a channel:

Rule 1. The renter should select a channel that interferes with the minimum number of channels being used by its neighbors.

After selecting a channel, the renter BS has to determine whether non-exclusive sharing of the selected channel is feasible using the following rule:

Rule 2. Non-exclusive spectrum sharing is feasible as long as the maximum achievable signal-to-interference ratio (SIR) on the selected channel is higher than the required SIR threshold of the network's supported services.

If non-exclusive sharing is feasible, the BS schedules data transmissions on the selected channel with appropriate transmission power control settings.

\subsubsection{Self-interference caused by resource renting}

The above Resource Renting mechanism may cause severe self-interference among overlapping cells. We use $r(x)$ to denote the minimum number of channels required by a BS $x$ 
to satisfy the QoS of its workload. Let $G(x)$ denote the grand candidate channel set for the renter BS $x$. In Figure 2.3, we illustrate a scenario with three overlapping cells $a, b$, and c. In this example, we have four channels in total, and $r(a)=r(b)=r(c)=2$. BS $a$ 's active channel set is $\{1,2\}$ and its candidate channel set is $\{3\}$. BS $c$ 's active channel set is $\{1,3\}$ and its candidate channel set is $\{2\}$. Since BS $b$ only has one active channel 4 , it needs more channels to satisfy the QoS of its workload. Its neighboring BSs, $a$ and $c$, have candidate channels to share with BS $b$. According to Rule 1, BS $b$ can select channel 2 in $G(b)$ to rent from BS $c$. Unfortunately, BS $b$ 's renting of channel 2 will cause interference to BS $a$ 's communications since BS $a$ also uses channel 2. In this case, self-interference between BS $a$ and BS $b$ may severely degrade the network performance of both cells.

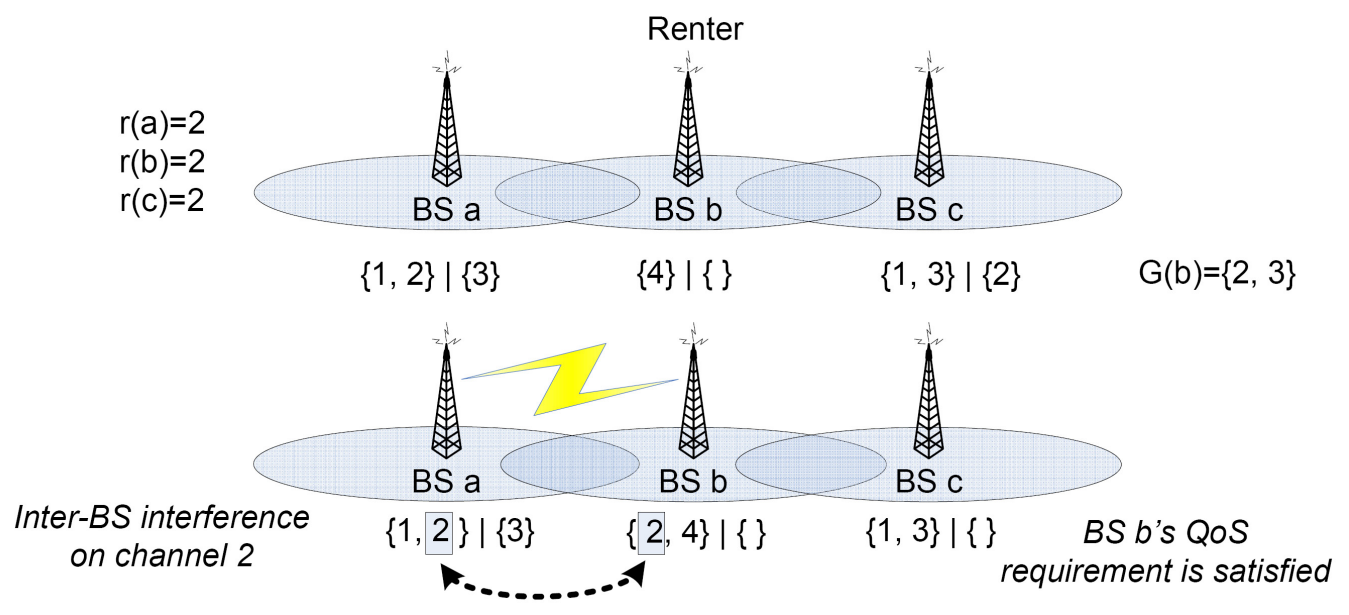

Figure 2.3: Inter-cell Resource Renting in 802.22.

\subsubsection{Exclusive Spectrum Sharing: ODSC}

To completely avoid self-interference, 802.22 also prescribes exclusive spectrum sharing of the selected channel (target channel) via ODSC [26]. The renter BS becomes the 
contention source when initiating the ODSC. The contention source randomly selects a channel contention number $(\mathrm{CCN})$ that is uniformly distributed in the range $[0, W-1]$, where $W$ is a constant representing the contention window size. The contention source includes its $\mathrm{CCN}$ and the target channel number in a spectrum contention request that it broadcasts to contention destination BSs. After receiving a spectrum contention request, a contention destination selects a $\mathrm{CCN}$ in the same manner as the contention source. Then the contention destination determines the outcome of the pair-wise contention using this contention resolution rule: the $\mathrm{BS}$ with a greater $\mathrm{CCN}$ value wins the pair-wise contention. This rule implies that the contention source's probability of winning a pair-wise contention is $1 / 2$. If the contention source wins the contended channel, all contention destinations perform channel switching to vacate the channel.

This contention rule implies the "absolute" fairness-i.e., that every BS has an equal probability of winning the target channel. In Chapter 6, we will present a contention protocol that ensures the weighted fairness such that a BS with higher QoS requirement or traffic load will have a greater probability of winning the target channel. 


\section{Chapter 3}

\section{Synchronous Channel Hopping Based}

\section{Rendezvous}

This chapter is organized as follows. In Sections 3.1 and 3.2, we describe the system model and the QCH system, respectively. We present two optimal synchronous QCH systems in Section 3.4. In Section 3.5, we compare our proposed QCH systems and existing $\mathrm{CH}$ schemes using simulation results. We conclude this chapter in Section 3.6.

\subsection{System Model}

We assume an OSS environment, where secondary users equipped with CRs dynamically access spectrum. Suppose multiple pairs of (secondary) senders and receivers are operating over $N$ orthogonal frequency channels that are licensed to the primary user. Channels are labeled as $0,1, \ldots, N-1$. Each node (a node may be a sender or a receiver) is equipped with a single half-duplex transceiver. Hence, a node can only listen to or transmit over 
one channel at a time. A packet sent over a channel can be heard by any node within the communication range of the transmitting node.

Time-slotted system. We consider a time-slotted communication system, where a global system clock exists. The local clock of each node may be synchronized to the global clock or may differ with the global clock by a certain amount of clock drift. A network node is assumed to be capable of hopping between different channels according to a channel hopping sequence and its local clock. A packet can be exchanged if the sender and the receiver hop onto the same channel at the same timeslot. We assume that one timeslot is long enough to exchange multiple packets.

Channel hopping (CH) sequence. A $\mathrm{CH}$ sequence (or a sequence) determines the order with which a node visits all available channels. We represent a $\mathrm{CH}$ sequence $u$ of period $T$ as a polynomial of order $(T-1)$ called the $\mathrm{CH}$ Sequence Function (CSF):

$$
u=f_{u}(x)=u_{0}+u_{1} x+u_{2} x^{2}+\ldots+u_{i} x^{i}+\ldots+u_{T-1} x^{T-1},
$$

where $u_{i} \in[0, N-1]$ represents the channel index of sequence $u$ in the $i^{\text {th }}$ timeslot of a $\mathrm{CH}$ period.

Given two sequences of period $T, u$ and $v$, and their CSFs, $f_{u}(x)$ and $f_{v}(x)$, if $\exists i \in$ $[0, T-1]$ s.t. $u_{i}=v_{i}=h$, where $h \in[0, N-1]$, we say that $u$ and $v$ rendezvous in the $i^{\text {th }}$ timeslot on channel $h$. The $i^{\text {th }}$ timeslot is called the rendezvous slot and channel $h$ is called the rendezvous channel between $u$ and $v$. Let $I_{h}(u, v)$ denote a function that indicates whether channel $h$ is a rendezvous channel between two sequences $u$ and $v$, i.e.,

$$
I_{h}(u, v)=\left\{\begin{array}{l}
1, \text { if } \exists i \in[0, T-1], \text { s.t. } u_{i}=v_{i}=h \\
0, \text { otherwise. }
\end{array}\right.
$$


The number of rendezvous channels between two sequences $u$ and $v, C(u, v)$, is defined as

$$
C(u, v)=\sum_{h=0}^{N-1} I_{h}(u, v) .
$$

Channel hopping systems. In the OSS paradigm, the value of $C(u, v)$ directly impacts the robustness of the pairwise control channel established using sequences $u$ and $v$. Recall that secondary users share spectrum opportunistically with incumbent users who have priority access rights. In such a scenario, secondary users are required to vacate the currently occupied channels when incumbent signals are detected in them. This requirement poses a difficult challenge in the design of MAC protocols for CR networks-in particular, in terms of how to establish control channels in such a way that enables the reliable exchange of control information despite the unpredictable appearance of incumbent signals. The robustness of the control channels established using sequences $u$ and $v$ is proportional to the value of $C(u, v)$, since this value determines the number of distinct channels in which the rendezvous occur within a sequence period. If the rendezvous are spread out over a greater number of distinct channels, then the probability of link breakage caused by the inability to exchange control packets (which in turn is due to the appearance of incumbent signals) decreases. Thus, we have the following channel hopping system design problem.

Problem 1. Given $T$, the CH system design problem is to devise a set of $C H$ sequences of period $T$, denoted as $H$, which satisfies the following two properties:

1. $\forall u \in H, u$ is a polynomial of order $(T-1)$;

2. $m \geq 1$, where $m=\min _{\forall u, v \in H}\{C(u, v)\}$. 
The set $H$ is called a $\mathrm{CH}$ system of period $T$, and $m$ is the degree of overlapping of the CH system $H$.

It is readily apparent that a $\mathrm{CH}$ system $H$ is a quorum system, since it satisfies the intersection property: any two sequences in $H$ have at least one overlap. Each $\mathrm{CH}$ sequence in $H$ is a quorum.

Coexistence with incumbent users. All secondary nodes coexist with primary users in the following way: a secondary node is able to detect the primary user signals using spectrum sensing techniques [1], and thus avoids transmitting on a channel where primary user signals are detected. The primary user signals may appear in any channel.

\subsection{The Quorum-based Channel Hopping System}

In this section, we introduce an algorithm that uses a quorum system to construct a $\mathrm{CH}$ system for any value of $m \in[1, N]$. We refer to this algorithm as Algorithm 1 .

Without loss of generality, suppose we want to construct a $\mathrm{CH}$ system where every pair of $\mathrm{CH}$ sequences rendezvous in $m$ different channels. We randomly select $m$ channels from $\{0, \ldots, N-1\}$ to construct a set of rendezvous channels, such as $R=$ $\left\{h_{0}, h_{1}, \ldots, h_{m-1}\right\}$. In our construction algorithm, every $\mathrm{CH}$ sequence is composed of $m$ frames and each frame is composed of $k$ slots ( $k$ is called the frame length). Hence, the period of each $\mathrm{CH}$ sequence is $T=m \cdot k$. We use the following example to explain the construction algorithm.

Suppose the set of rendezvous channels is $R=\{0,1,2\}$, each $\mathrm{CH}$ sequence is composed of $m=3$ frames, and each frame has $k=3$ slots. 
1. First construct a universal set, $U=\mathbf{Z}_{k}=\{0,1,2\}$;

2. Construct a quorum system $S$ under $U, S=\{\{0,1\},\{0,2\},\{1,2\}\} ;{ }^{1}$

3. Using the quorum $q_{0}=\{0,1\} \in S$, we construct a $\mathrm{CH}$ sequence $u$ using the following procedure.

- We make $k$ channel assignments for the first frame in $u$ using the following equation:

$$
u_{i}=\left\{\begin{array}{l}
h_{0}, \text { if } i \in q_{0}, \\
h, \text { if } i \notin q_{0} .
\end{array}\right.
$$

where $h$ is a randomly selected channel from $\{0, \ldots, N-1\}$. The (timeslot, channel) assignments for the first frame is obtained using a channel in $R, h_{0}=$ 0 -i.e., $u_{0}=0, u_{1}=0, u_{2}=h$.

- Repeat the above procedure to make (timeslot, channel) assignments for each of the other frames using the remaining channels in $R$ (i.e., channels 1 and 2 in this example). The resulting $\mathrm{CH}$ sequence is $u=\left\{0+0 \cdot x+h \cdot x^{2}+1\right.$. $\left.x^{3}+1 \cdot x^{4}+h \cdot x^{5}+2 \cdot x^{6}+2 \cdot x^{7}+h \cdot x^{8}\right\} ;$

4. Repeat Step (3) for each of the other quorums in $S$ (i.e., $q_{1}=\{0,2\}$ and $q_{2}=\{1,2\}$ ) to construct two other sequences, $v$ and $w$. The three $\mathrm{CH}$ sequences-u, $v, w$-are the elements of the set $Q$, which contains $|S|=3 \mathrm{CH}$ sequences.

The sequences in $Q$ are illustrated in Figure 3.1. Algorithm 1 constructs each sequence in $Q$ by making $k$ (timeslot, channel) assignments for each of the $m$ rendezvous channels. One quorum in $S$ is needed to generate each CH sequence in $Q$. Thus, $|Q|=|S|$.

\footnotetext{
${ }^{1}$ The desired properties of the $\mathrm{CH}$ system determines the particular quorum system that is constructed. In Section 3.4, we discuss a number of quorum systems that can be used to construct optimal CH systems.
} 
Note that $\forall u, v \in Q$, there are two corresponding quorums $p, q \in S$ used for constructing $u$ and $v$, respectively. Because of the intersection property of $S, u$ and $v$ overlap in exactly $m$ distinct channels_-viz, the channels $h_{d} \in R, d \in[0, m-1]$ (see lines 3 and 6 of Algorithm 1). Also note that all of the sequences in $Q$ have the same period, viz, $T=m \cdot k$ slots. Therefore, $Q$ is a $\mathrm{CH}$ system that satisfies the requirements of Problem 1 . We refer to the $\mathrm{CH}$ system constructed using Algorithm 1 as a quorum-based channel hopping $(Q C H)$ system.

In the next few sections, we will show how to minimize TTR between any two $\mathrm{CH}$ sequences. We will also describe a scheme that addresses the rendezvous convergence problem of $\mathrm{CH}$ systems by selecting a particular type of quorum system used in Algorithm 1.

\begin{tabular}{|c|c|c|c|c|c|c|c|c|}
\hline Slot Index: & 0 & 2 & 3 & 4 & 5 & 6 & 7 & 8 \\
\hline u & 40 & 2 & 1 & 1 & 0 & 2 & 2 & 1 \\
\hline $\mathbf{v}$ & 0 & 0 & 1 & 2 & 1 & $\sqrt{2}$ & 0 & 2 \\
\hline $\mathbf{w}$ & $\checkmark 0$ & $\mathbf{0}$ & 2 & $\checkmark 1$ & 1 & 0 & $\sqrt{2}$ & 2 \\
\hline
\end{tabular}

Figure 3.1: An illustration of QCH system $Q$ with $m=3$ and $k=3$. Any two sequences overlap on three channels. We use the quorum system $S=\{\{0,1\},\{0,2\},\{1,2\}\}$ over $U=\{0,1,2\}$ to construct $Q$. The numbers inside the slots denote channel index values: bold-font values denote channel indexes from $R$ and the non-bold font values denote channel indexes randomly chosen from $\{0, \ldots, N-1\}$.

\subsection{Metrics for Evaluating CH Systems}

We introduce two metrics-maximum time-to-rendezvous (MTTR) and load-that are used to evaluate $\mathrm{CH}$ systems. Note that both metrics can be used to evaluate all $\mathrm{CH}$ 


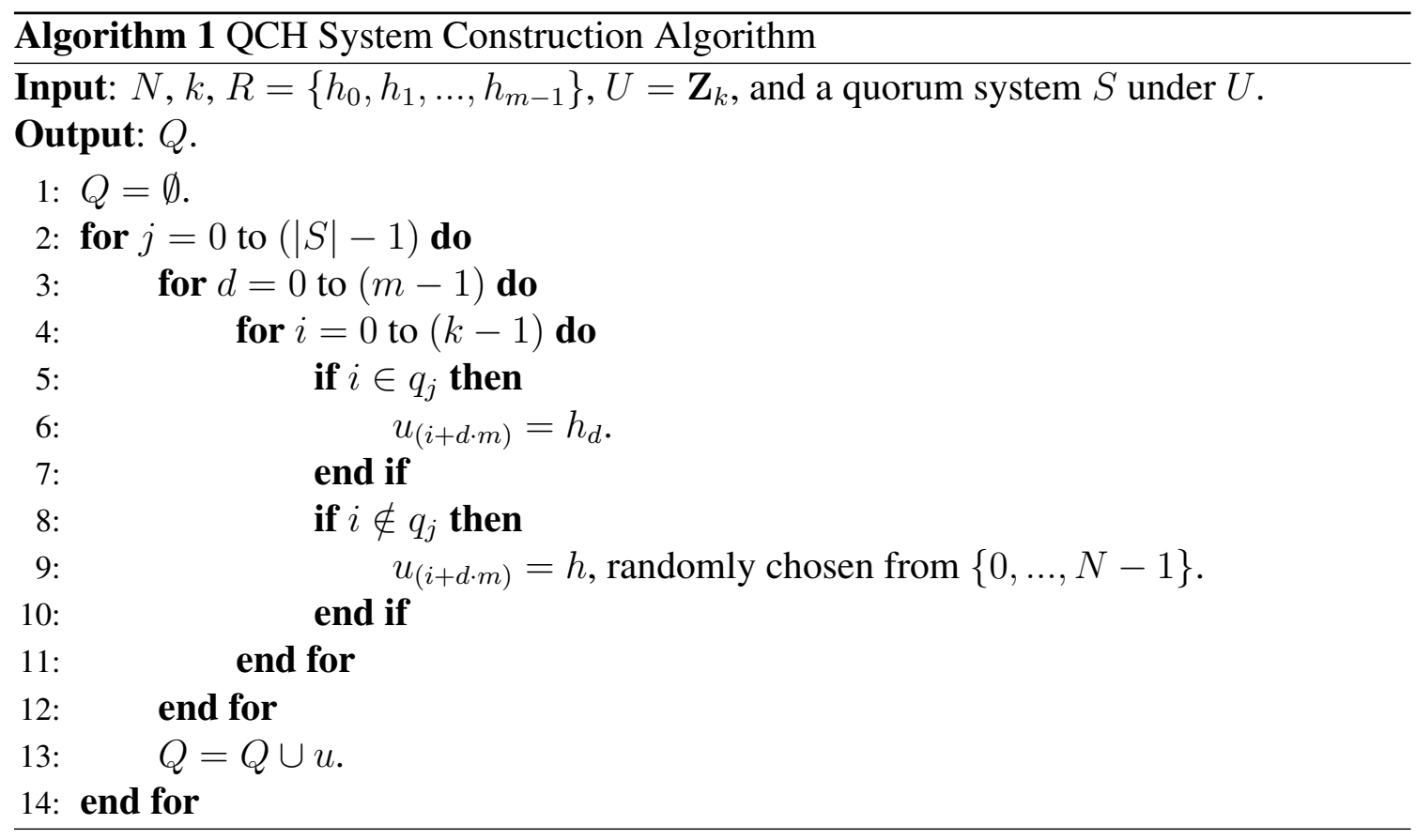

systems, not just quorum-based $\mathrm{CH}$ systems.

\subsubsection{Maximum Time-to-Rendezvous}

The first metric we introduce is the maximum time-to-rendezvous (MTTR) for a CH system, which is defined as the maximum time for any pair of sequences in a $\mathrm{CH}$ system to rendezvous. Let $M(H)$ denote the MTTR of a CH system $H$. It is obvious that the MTTR value impacts the medium access delay of MAC protocols that utilize channel hopping since the exchange of control information is not possible without rendezvous. Networks that require stringent delay requirements will require a $\mathrm{CH}$ system with a small MTTR value. For example, in a mobile CR network, neighboring nodes have to exchange timesensitive control information frequently-information such as spectrum sensing related control information, node location, link connectivity, etc. 
In a QCH system $Q$ constructed using Algorithm 1, the MTTR value is equal to the frame length $k$. In the QCH system $Q$ given in Figure 3.1, the MTTR is $M(Q)=3$. In Section 3.4.1, we describe a methodology for designing a QCH system that is optimal in terms of minimizing the MTTR.

\subsubsection{Load of CH Systems}

Load of Quorum Systems. Here, we provide some definitions regarding the load of quorum systems. We will utilize these definitions to describe one of the optimal QCH systems introduced in Section 3.4. In the context of quorum systems, a strategy is a rule giving each quorum an access frequency so that the frequencies sum up to one. In other words, a strategy gives the frequency of picking each quorum. A strategy induces a load on each element, which represents the fraction of the time the element is used. Specifically, an element's load is the summation of the frequencies of all quorums that the element belongs to. Below, we provide more precise definitions.

Definition 4. Let a quorum system $S=\left\{q_{0}, q_{1}, \ldots, q_{\kappa-1}\right\}$ be given over a universal set $U$. Then $W \in[0,1]^{\kappa}$ is a strategy for $S$ if it is a probability distribution over the quorums $q_{j} \in S$, i.e., $\sum_{j=0}^{\kappa-1} W_{j}=1$.

The system load, $\mathcal{L}(S)$, is the minimal load on the busiest element, minimizing over the strategies. A more precise definition follows.

Definition 5. Let a strategy $W$ be given for a quorum system $S=\left\{q_{0}, q_{1}, \ldots, q_{\kappa-1}\right\}$ over a universal set $U$. For an element $i \in U$, the load induced by $W$ on $i$ is

$$
l_{W}(i)=\sum_{q_{j} \in S: i \in q_{j}} W_{j}
$$


The load induced by a strategy $W$ on a quorum system $S$ is

$$
\mathcal{L}_{W}(S)=\max _{i \in U} l_{W}(i)
$$

The system load on a quorum system $S$ is

$$
\mathcal{L}(S)=\min _{W}\left\{\mathcal{L}_{W}(S)\right\}
$$

where the minimum is taken as the system load over all strategies $W$.

In $\mathrm{CH}$ protocols, spreading out the rendezvous in time and frequency (i.e., channels) is important in order to take full advantage of the frequency diversity of multi-channel medium access. If a large proportion of neighboring nodes rendezvous on the same channel, then channel congestion can occur and lead to a control channel bottleneck problemwe use the term rendezvous convergence to refer to such a problem. Some $\mathrm{CH}$ protocols (e.g., SSCH [4]) implement "customized" mechanisms to prevent rendezvous convergence. Ideally, a $\mathrm{CH}$ protocol should spread out the rendezvous over all channels evenly.

One advantage of using the proposed approach to devise $\mathrm{CH}$ schemes is that it can formally characterize the rendezvous convergence problem using the measure of load which is used in the study of quorum systems. In quorum systems, a strategy is a probabilistic rule that gives the frequency of accessing each quorum so that the frequencies sum up to one. Since a $\mathrm{CH}$ system is in essence a quorum system, we can use the definition of load given above to create an analogous definition for the load of a $\mathrm{CH}$ system. Let $W_{0}$ denote the following strategy: each radio randomly selects a sequence from a $\mathrm{CH}$ system with equal probability. Given a $\mathrm{CH}$ system, $H$, the load of $H$ induced by $W_{0}, \mathcal{L}_{W_{0}}(H)$, is the load of the busiest element induced by $W_{0}$; the busiest element, in this context, refers to the (timeslot, channel)-pair included in the largest number of $\mathrm{CH}$ sequences. We define 
the load of a CH system as the load of the $\mathrm{CH}$ system induced by the particular strategy $W_{0}$.

In the QCH system $Q$ shown in Figure 3.1, the load is $\mathcal{L}_{W_{0}}(Q)=2 / 3$. In Section 3.4, we will discuss a $\mathrm{QCH}$ design that is optimal in the sense that it minimizes the load of the QCH system.

\subsection{Optimal Synchronous QCH Systems}

In this section, we describe two optimal QCH systems, both of which require global clock synchronization.

\subsubsection{Minimizing the MTTR in QCH Systems}

Minimizing the MTTR of a QCH system is equivalent to minimizing its frame length $k$. To design an optimal QCH system that minimizes the MTTR, we first need to solve the following problem:

Problem 2. Given a QCH system $Q$,

$$
\begin{aligned}
& \text { minimize } k, \\
& \text { subject to } \mathcal{L}_{W_{0}}(Q)<1 .
\end{aligned}
$$

The constraint $\mathcal{L}_{W_{0}}(Q)<1$ equates to $\bigcap_{u \in Q} u=\emptyset$, which is needed to avoid the scenario in which the load of the QCH system is equal to one (i.e., there is at least one (timeslot, channel)-pair that is included in all of the sequences in $Q$ ).

The lower bound for $k$. To solve Problem 2, we find the lower bound for $k$ when the load of the QCH system is less than one in the following theorem. 
Theorem 1. Given a $Q C H$ system $Q$, a necessary condition for $\mathcal{L}_{W_{0}}(Q)<1$ is $k \geq 3$.

Proof. We prove this theorem by contradiction. Let $k \leq 2$ and suppose we have a QCH system $Q$, where $T=k \cdot m$ and $\mathcal{L}_{W_{0}}(Q)<1$.

If $k=1$, then $T=m$. Since

$$
C(u, v) \geq m \geq 1, \forall u, v \in Q,
$$

all sequences in $Q$ must be identical. In this case, the load of $Q$ is 1 , which contradicts the constraint $\mathcal{L}_{W_{0}}(Q)<1$.

If $k=2$, the universal set is $U=\{0,1\}$ according to Algorithm 1 . Any quorum system $S$ over $U=\{0,1\}$ has a system load of one, i.e., $\mathcal{L}(S)=1$. Since the QCH system $Q$ is constructed using $S$,

$$
\mathcal{L}_{W_{0}}(Q) \geq \mathcal{L}(S)=1,
$$

and this contradicts the constraint $\mathcal{L}_{W_{0}}(Q)<1$.

From Figure 3.1, we can see that there exists a QCH system in which $k=3$ and its load is less than one.

From the above arguments, it is clear that $k \geq 3$ is a necessary condition for $\mathcal{L}_{W_{0}}(Q)<$ 1.

Construction of the M-QCH system. The QCH system that achieves the lower bound for $k$ (i.e., $k=3$ ) is an optimal QCH design in the sense that it minimizes the MTTR while keeping the load less than one. We refer to such a system as an M-QCH System, and it can be constructed using Algorithm 1 with a majority cyclic quorum system over a universal set $U=\mathbf{Z}_{3}$. The example $\mathbf{Q C H}$ system shown in Figure 3.1 is an $\mathbf{M}-\mathbf{Q C H}$ system. An M-QCH system can support $m$ rendezvous channels $(m \in[1, N])$ and it has the lowest 
MTTR value (i.e., 3) among all QCH systems. Hence, M-QCH systems are advantageous in establishing control channels with minimal medium access delay.
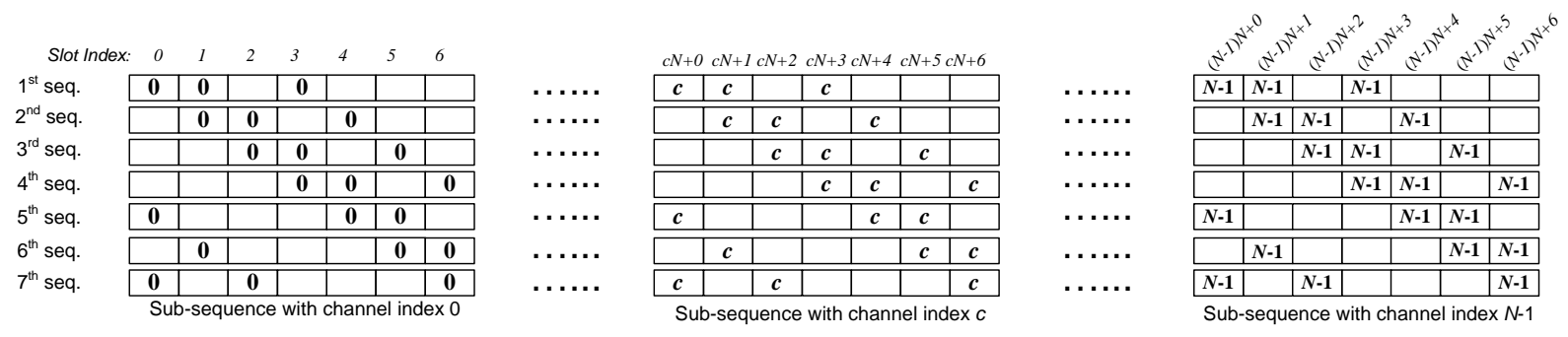

Figure 3.2: An L-QCH system, $Q$, with $m=N$ rendezvous channels and $\tau=7$. To construct $Q$, we use a minimal cyclic quorum system $S=\{\{0,1,3\},\{1,2,4\}$, $\{2,3,5\},\{3,4,6\},\{4,5,0\},\{5,6,1\},\{6,0,2\}\}$ under $U=\mathbf{Z}_{7}$. The channel indexes for "blank" slots are randomly selected from $[0, N-1]$. Any two sequences in this system overlap on all $N$ channels.

\subsubsection{Minimizing the Load}

In this subsection, we study an optimal $\mathrm{QCH}$ system, $Q$, that has the minimal load under the constraint $M(Q) \leq \tau$, for a given value of $\tau$. To construct such a QCH system, we need to solve the following problem:

Problem 3. Given a $Q C H$ system $Q$,

$$
\begin{aligned}
& \operatorname{minimize} \mathcal{L}_{W_{0}}(Q), \\
& \text { subject to } M(Q) \leq \tau,
\end{aligned}
$$

where $\tau$ is the maximum allowed MTTR of $Q$.

The lower bound for load. We solve Problem 3 by finding the lower bound for $\mathcal{L}_{W_{0}}(Q)$ under the constraint $M(Q) \leq \tau$. 
Theorem 2. Given a $Q C H$ system $Q$ where $M(Q) \leq \tau$, the minimum load induced by $W_{0}$ on $Q$ is $\frac{1}{\sqrt{\tau}}$, i.e.,

$$
\mathcal{L}_{W_{0}}(Q) \geq \frac{1}{\sqrt{\tau}}
$$

Proof. In a QCH system $Q$ where $M(Q) \leq \tau$, since $M(Q)=k$, we have $k \leq \tau$ ( $k$ is the frame length).

According to Algorithm 1, such a QCH system $Q$ is constructed using a quorum system $S$ over $U=\mathbf{Z}_{k}$. Thus, we have $\mathcal{L}_{W_{0}}(Q) \geq \mathcal{L}(S)$.

The propositions 4.1 and 4.2 in [46] state that the following relation is true:

$$
\mathcal{L}(S) \geq \max \left\{\frac{1}{\varphi(S)}, \frac{\varphi(S)}{k}\right\}
$$

where $\varphi(S)$ is the size of the smallest quorum in the quorum system $S$. Using the inequality of arithmetic and geometric means, it can be shown that

$$
\mathcal{L}(S) \geq \frac{1}{\sqrt{k}}
$$

Since $k \leq \tau$, we have $\mathcal{L}_{W_{0}}(Q) \geq \mathcal{L}(S) \geq \frac{1}{\sqrt{k}} \geq \frac{1}{\sqrt{\tau}}$.

Construction of the L-QCH system. Using Algorithm 1, we can construct an optimal QCH system that minimizes the value of $\mathcal{L}_{W_{0}}(Q)$ by using a minimal cyclic quorum system over a universal set $U=\mathbf{Z}_{\tau}$. We refer to such an optimal QCH system as an $L$ QCH System. An L-QCH system includes $\tau$ unique $\mathrm{CH}$ sequences. The L-QCH system $Q$ shown in Figure 3.2 is constructed using a minimal cyclic quorum system $S$ over $U=\mathbf{Z}_{7}$, which has a degree of overlapping of $m=N$ and $\tau=7$. The load $\mathcal{L}_{W_{0}}(Q)=\frac{3}{7} \approx \frac{1}{\sqrt{\tau}}$.

A comparison of all the synchronous $\mathrm{CH}$ schemes discussed in this chapter are summarized in Table 3.1 . 
Table 3.1: A comparison of synchronous $\mathrm{CH}$ schemes.

\begin{tabular}{|c||c|c|c|}
\hline & $\begin{array}{c}\text { Degree of } \\
\text { overlapping }\end{array}$ & MTTR & Load \\
\hline \hline SSCH & 1 & $N+1$ & $\frac{1}{N-1}$ \\
\hline M-QCH & {$[1, N]$} & 3 & $\frac{2}{3}$ \\
\hline L-QCH & {$[1, N]$} & $k$ & $\frac{1}{\sqrt{\tau}}$ \\
\hline
\end{tabular}

\subsection{Performance Evaluation}

We simulate the QCH scheme in ns-2 (version 2.31) [63] and use three MAC-layer reference protocols for comparison: IEEE $802.11 \mathrm{~b}, \mathrm{SSCH}$, and the SeqR protocol. The data rate is $11 \mathrm{Mbps}$ by default. Note that IEEE $802.11 \mathrm{~b}$ and SSCH were not designed for use in CR networks. However, they serve as good benchmarks in evaluating the performance of $\mathrm{QCH}$. Furthermore, the design criteria of $\mathrm{CH}$ schemes for conventional multi-channel networks are almost identical to those of $\mathrm{CH}$ schemes for $\mathrm{CR}$ networks, and $\mathrm{SSCH}$ is one of the most well-known schemes of the former type. In the simulations, secondary nodes can opportunistically access three channels (i.e., $N=3$ ). The channel switching delay is chosen as $80 \mu \mathrm{s}$, which is well supported by existing technology [23]. The duration of a time slot is 200 ms. Every node uses Ad hoc On-Demand Distance Vector Routing (AODV) [47] as the routing protocol. At the transport layer, UDP is used in the simulations by default. The traffic generator uses Constant Bit Rate (CBR) flows with a flow rate of $11 \mathrm{Mbps}$ and a packet size of 512 bytes. The transmission range of every node is $250 \mathrm{~m}$. We simulated two networks: a static network with ten single-hop flows in a $100 \mathrm{~m} \times 100 \mathrm{~m}$ square area and a random network with five multi-hop flows in a $1000 \mathrm{~m} \times 1000 \mathrm{~m}$ square area. In the simulations, we study the time-to-rendezvous (TTR) value and the throughput in each $\mathrm{CH}$ protocol under varying conditions, including random incumbent traffic, node 
mobility, and multi-flow complex networks.

The following protocols were simulated.

- SSCH: Each node randomly chooses one (channel, seed)-pair to construct its $\mathrm{CH}$ sequence. For example, if a node selects the pair $(0,1)$, then its $\mathrm{CH}$ sequence has a period of $(N+1)$ slots, and the channel indexes in its sequence are $\{0,1,2,1\}$. The last slot of a period is the parity slot, and the channel index of this slot is equal to the value of the node's seed.

- SeqR: This is the protocol proposed in [19]; it was briefly described in Section 2.

- QCH: We simulate two synchronous QCH systems (M-QCH, and L-QCH).

We assume that every node randomly picks one sequence from a $\mathrm{CH}$ system and performs channel hopping in accordance with the sequence. Once the sending-receiving node pair rendezvous on a channel, the pair performs common hopping to exchange data packets. The sender follows the receiver's sequence.

Incumbent traffic generation. In the simulations, we generated incumbent traffic as follows. In every time slot, the incumbent transmitter decides whether to transmit or not by flipping a coin. If the incumbent transmitter decides to transmit, it randomly selects one or two channels and transmits packets in the current time slot. All of the secondary nodes are within the transmission range of the incumbent transmitter. A single incumbent transmitter was simulated. A channel is tagged as "unavailable" while incumbent traffic is present on it. All secondary nodes should refrain from transmitting on unavailable channels during the period of incumbent transmission. Note that all nodes that perform channel hopping are secondary nodes. 


\subsubsection{Impact of Time-to-Rendezvous}

We first simulated a single-hop flow to show the effect of TTR on channel access delay and the effect of channel switching overhead on throughput. The results are shown in Figure 3.3. We can see that the starting times of the traffic delivery for the simulated protocols are different, which coincides with the discrepancy of the protocols' channel access delays due to the variation in TTR values. Note that the throughput of each $\mathrm{CH}$ protocol is lower than that of $802.11 \mathrm{~b}$, which we can attribute to the channel switching overhead.

Next, we simulated a network with ten single-hop disjoint flows in a $100 \mathrm{~m} \times 100 \mathrm{~m}$ square area. Two flows are considered disjoint if they do not share either endpoint. The average TTR for three $\mathrm{CH}$ schemes (when there is no incumbent traffic) is shown using the leftmost group of bars in Figure 3.4. As can be seen, M-QCH has the lowest average TTR compared to SSCH and SeqR - this is expected since M-QCH has the lowest MTTR value among the three $\mathrm{CH}$ protocols.

\subsubsection{Impact of Degree of Overlapping}

As expected, our simulation results indicate that a $\mathrm{CH}$ scheme's degree of overlapping has a clear impact on its TTR value when the incumbent transmitter is active. The average TTR for three $\mathrm{CH}$ schemes in the presence of incumbent traffic is shown using the center and rightmost groups of bars in Figure 3.4. M-QCH has a clear advantage over SSCH and SeqR in terms of TTR, because M-QCH's degree of overlapping is greater than that of the other two schemes in this simulation. This advantage becomes more evident in the presence of incumbent traffic since a pair of nodes using $\mathrm{M}-\mathrm{QCH}$ can rendezvous on other 


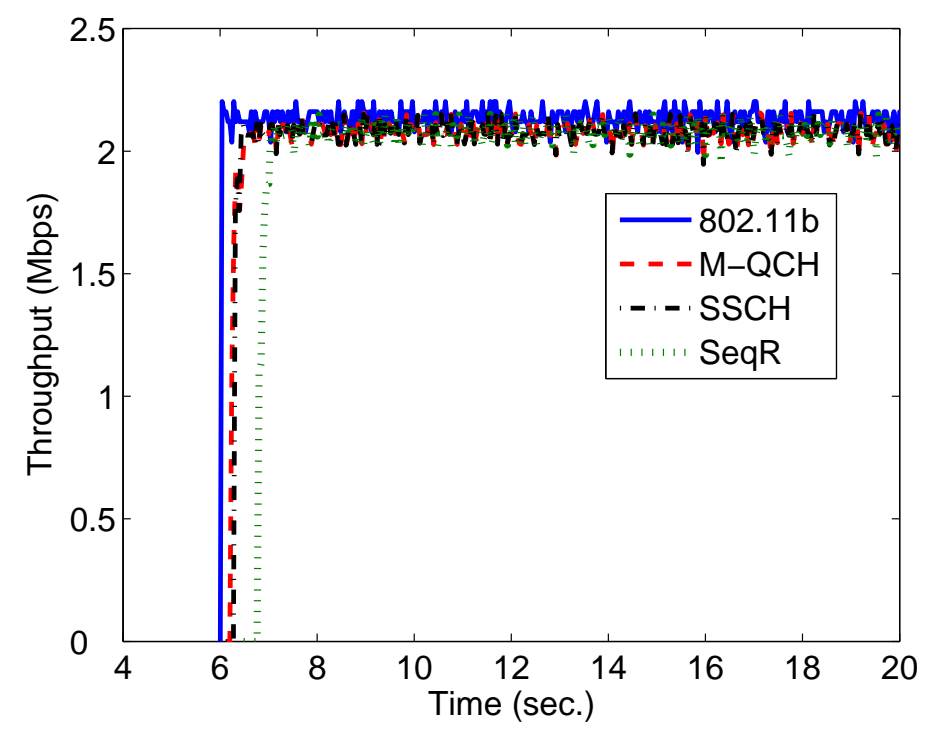

Figure 3.3: Effect of time-to-rendezvous and channel switching.

channels if the current rendezvous channel is occupied by incumbent signals. In contrast, a pair of nodes using either SSCH or SeqR can rendezvous only on one channel (here, we are referring to the initial rendezvous). This implies that the nodes may not be able to achieve the initial rendezvous until the incumbent vacates the rendezvous channel. Note that in $\mathrm{SSCH}$, initial rendezvous is needed to exchange data, such as each other's sequence, that is required to rendezvous in multiple channels.

Next, we set up ten non-disjoint flows in a $100 m \times 100 m$ square area, where every node serves as both a transmitter and a receiver in multiple flows. In other words, this scenario includes multiple simultaneous flows with common endpoints. We assume temporary common hopping, i.e., each transmitter node has to change its hopping sequence and follow the receiver's sequence after a rendezvous has occurred to bootstrap communications. If the receiver node also acts as a transmitter in another flow, it must also follow the sequence of its intended receiver after a rendezvous. Thus, some nodes in this network 


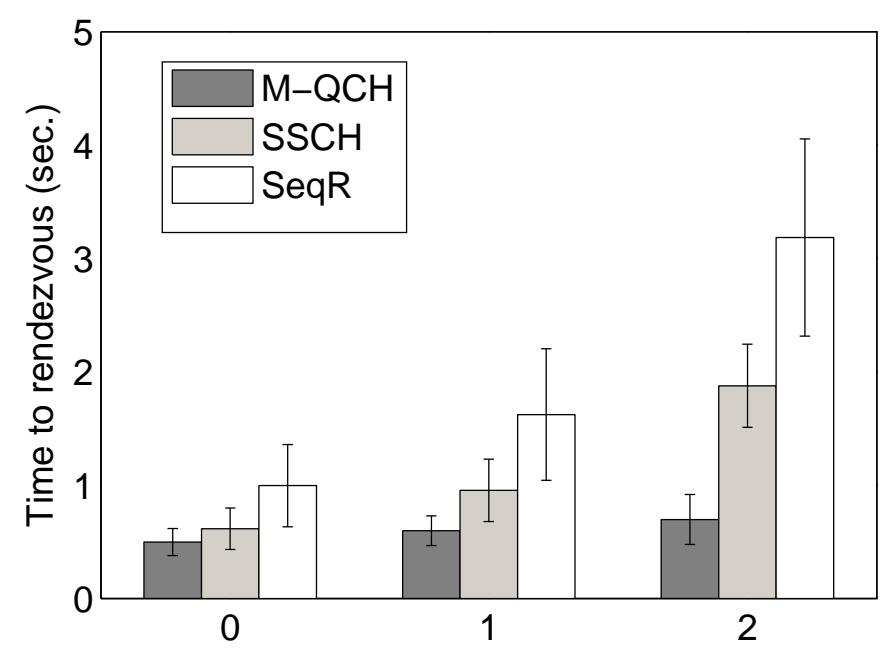

Number of channels selected by the incumbent transmitter

Figure 3.4: Time-to-rendezvous with incumbent traffic.

have to switch between $\mathrm{CH}$ sequences continuously, and this scenario puts stress on a $\mathrm{CH}$ protocol's ability to establish links. Temporary common hopping prescribes that a transmitter should return back to its original sequence once the transmission has finished-this avoids the global synchronization of the $\mathrm{CH}$ sequences over the entire network (i.e., a scenario in which every node uses the same sequence). We compare the per-flow throughput of $\mathrm{M}-\mathrm{QCH}, \mathrm{SSCH}$, and SeqR in Figure 3.5. When an incumbent signal is detected, MQCH replaces the incumbent-occupied channel in its sequence with any incumbent-free channel. The results in the figure show that M-QCH outperforms the other two protocols, since M-QCH is faster in re-establishing links - this, of course, is due to the fact that $\mathrm{M}-\mathrm{QCH}$ enables rendezvous in a greater number of distinct channels per sequence period. 


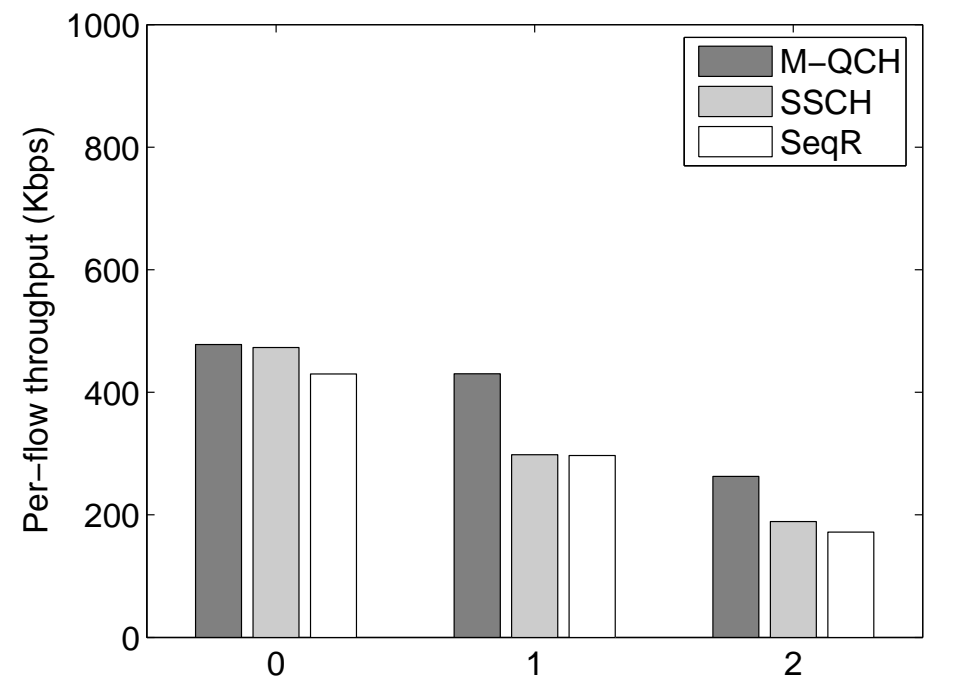

Number of channels selected by the incumbent transmitter

Figure 3.5: Throughput of non-disjoint flows with incumbent traffic.

\subsubsection{Impact of the Load}

In this set of simulation experiments, we investigated the effect of load (as defined in Section 3.3.2) on network performance. In general, the load of a $\mathrm{CH}$ system determines the number of concurrent co-channel transmissions in each time slot. If the load is low, the number of concurrent co-channel transmissions in each slot is small, which means that the traffic is more evenly distributed among different channels in every time slot. In general, a more even distribution of traffic among channels implies higher network throughput. In the simulations, we varied the number of disjoint flows in a $100 m \times 100 m$ square area, and the results are shown in Figure 3.6. In the figures, we can see that L-QCH and SeqR outperform the other schemes since the two schemes have the lowest load (viz $\frac{1}{3}$ ) compared to the other schemes (M-QCH has a load of $\frac{2}{3}$ and SSCH incurs a load of $\frac{1}{2}$ as indicated in Table 3.1). It is interesting to note that M-QCH's performance is inferior to that of the 


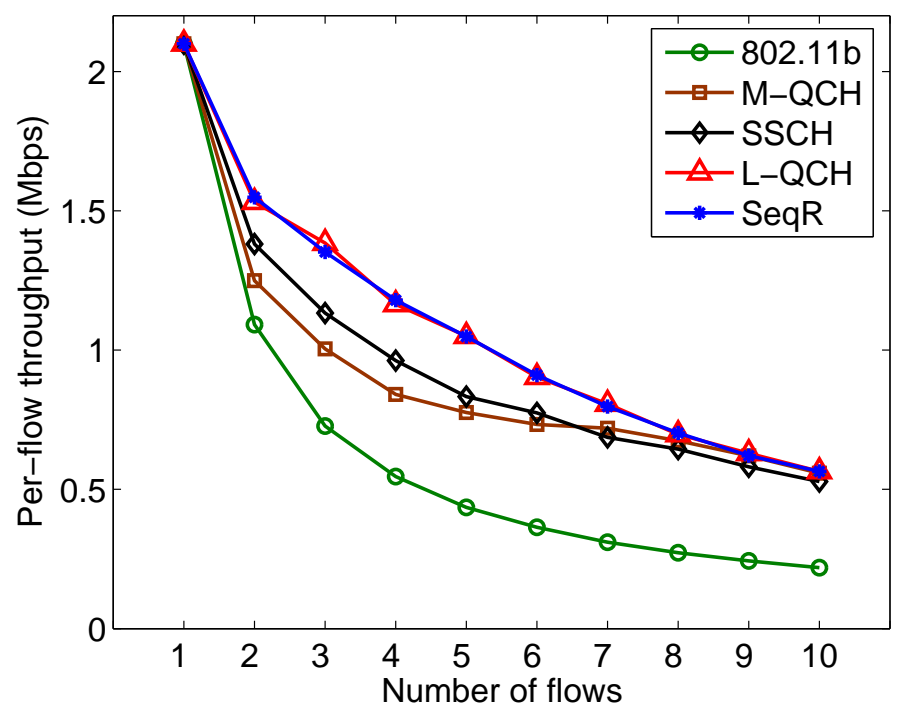

(a) Per-flow throughput;

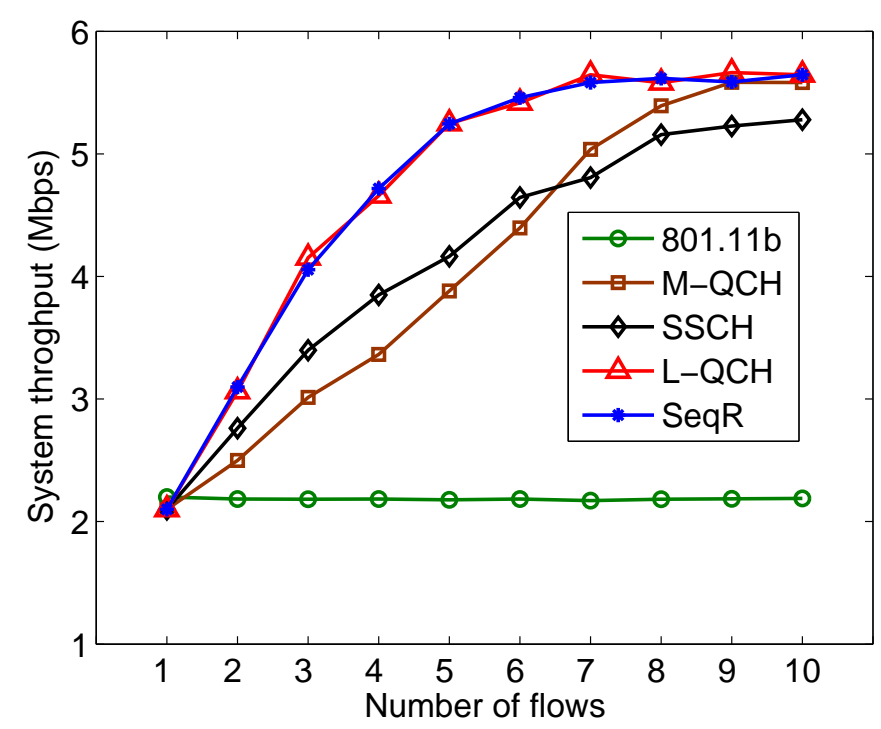

(b) System throughput.

Figure 3.6: Effect of load on throughput. 
other schemes when the number of flows is small (because M-QCH has the highest load); however, when the number of flows is large, the system throughput for SSCH is lower than that of the other schemes. This phenomenon can be attributed to the limiting effect of the parity slot prescribed by SSCH when the network is close to saturation: nodes using SSCH can only utilize $(N-1)$ channels specified by $(N-1)$ seed values in the parity slot. In contrast, nodes using the other $\mathrm{CH}$ protocols can fully utilize all $N$ channels in any time slot. From Figure 3.6, we can conclude that $\mathrm{CH}$ schemes that have lower load values are generally advantageous in terms of being able to support higher throughput; when the network is nearly saturated, the system throughput is closely related to the number of channels that can be fully utilized by each $\mathrm{CH}$ scheme.

\subsection{Summary}

In this chapter, we presented a systematic approach, based on quorum systems, for designing and analyzing channel hopping $(\mathrm{CH})$ protocols that enable the rendezvous (or control) channel establishment in CR networks. A noteworthy feature of the proposed Quorumbased Channel Hopping (QCH) system is that it can establish rendezvous (or control) channels in multiple frequency channels so that the secondary network is less vulnerable to the unpredictable appearance of incumbent signals. We proposed two synchronous designs of the QCH system: the first design minimizes the MTTR of the CH system and the second design guarantees the even distribution of the rendezvous points in terms of both time and frequency. Minimizing the MTTR ensures short expected TTR which decreases channel access delay. An even distribution of rendezvous points alleviates the rendezvous convergence problem. We have shown, using analytical and simulation results, that the 
$\mathrm{CH}$ schemes designed using the quorum-based framework outperform existing schemes under various network conditions. 


\section{Chapter 4}

\section{Asynchronous Channel Hopping Based Rendezvous}

In Chapter 3, by utilizing the intersection property of quorum systems, a set of $\mathrm{CH}$ sequences can be generated such that any pair selected from the set is guaranteed to rendezvous when the boundaries of the two selected $\mathrm{CH}$ sequences are synchronized. However, merely relying on the intersection property of quorum systems is not sufficient to guarantee rendezvous when the two $\mathrm{CH}$ sequences are asynchronous (i.e., the boundaries of the two $\mathrm{CH}$ sequences are misaligned).

The asynchronous channel hopping based rendezvous schemes can be classified into two categories.

- Asymmetric design: every node has a priori knowledge of its role as either a sender or a receiver (e.g., Bluetooth pairing). The sender and receiver nodes use different methods to generate their $\mathrm{CH}$ sequences for achieving rendezvous. 
- Symmetric design: every node behaves identically (i.e., a node may be either a sender or a receiver) and follows the same method for generating its $\mathrm{CH}$ sequence.

Since the symmetric design is independent of the assumption that every node has a priori knowledge of its role as either a sender or a receiver, it has a wider variety of applications than the asymmetric design. Note that a $\mathrm{CH}$ scheme can be either a symmetric or an asymmetric design depending on whether the sender and receiver employ different $\mathrm{CH}$ sequence generation algorithms or hop at different rates. For example, if every node has the same hopping rate $[54,60]$ or the same sequence-generation algorithm $[4,7,19,56]$, then the $\mathrm{CH}$ scheme is a symmetric design. In contrast, if the sender and receiver have different hopping rates [58] or employ different sequence-generation algorithms [6], then the $\mathrm{CH}$ scheme is an asymmetric design.

This chapter is organized as follows. We define the asynchronous channel hopping System in Section 4.1. In Sections 4.2 and 4.3, we describe two asymmetric ACH systems that are constructed by cyclic quorum systems and array-based quorum systems, respectively. We formulate the optimal $\mathrm{ACH}$ system design problem in Section 4.4. In Sections 4.5 and 4.6, we present an asymmetric optimal ACH system and a symmetric optimal ACH system. We analytically compare the proposed $\mathrm{ACH}$ system designs with existing $\mathrm{CH}$ schemes in Section 4.7. We evaluate our proposed $\mathrm{ACH}$ schemes using simulation results in Section 4.8, and summarize this chapter in Section 4.9.

\subsection{Asynchronous Channel Hopping System}

We first introduce the concepts of cyclic rotation and the rotation closure property in the context of quorum systems. Given a non-negative integer $k$ and a quorum $q$ in a quorum 
system $Q$ under the universal set $U=\{0, \ldots, n-1\}$, we use rotate $(q, k)$ to denote a $c y c l i c$ rotation to quorum $q$ :

$$
\operatorname{rotate}(q, k)=\{(i+k) \bmod n \mid i \in q\}
$$

For example, suppose we have a quorum system $Q=\{\{0,1\},\{1,2\}\}$ under $U=\mathbf{Z}_{3}$. For quorum $q=\{0,1\}$ and $k=2$, rotate $(q, k)=\{2,0\}$. Now let us define the rotation closure property of quorum systems.

Definition 6. A quorum system $Q$ over $U=\mathbf{Z}_{n}$ is said to have the rotation closure property if the following holds:

$$
\forall p, q \in Q, \forall k \in[0, n-1], \operatorname{rotate}(p, k) \cap q \neq \emptyset .
$$

For the sake of simplicity, we represent a $\mathrm{CH}$ sequence $u$ of period $T$ as a set of channel indexes:

$$
u=\left\{u_{0}, u_{1}, \ldots, u_{i}, \ldots, u_{T-1}\right\}
$$

where $u_{i} \in[0, N-1]$ represents the channel index of sequence $u$ in the $i^{\text {th }}$ timeslot of a $\mathrm{CH}$ period.

We extended the concept of cyclic rotation so that it is applicable to $\mathrm{CH}$ sequences. Given a $\mathrm{CH}$ sequence $u$, we use rotate $(u, k)$ to denote a cyclic rotation of $\mathrm{CH}$ sequence $u$ by $k$ timeslots, i.e.,

$$
\text { rotate }(u, k)=\left\{v_{0}, \ldots, v_{j}, \ldots, v_{T-1}\right\}
$$

where $v_{j}=u_{(j+k) \bmod T}, j \in[0, T-1]$ and $k$ is a non-negative integer. For example, given $u=\{0,1,2\}$ and $T=3$, rotate $(u, 2)=\{2,0,1\}$. 
A channel hopping $(\mathrm{CH})$ system of period $\mathrm{T}$ is defined as a set of $\mathrm{CH}$ sequences of period $T$. Here, we formally define an asynchronous channel hopping (ACH) system below.

Definition 7. An asynchronous channel hopping (ACH) system of period $T$ is a CH system of period $T$, which satisfies the rotation closure property: it consists of $\mathrm{CH}$ sequences such that any two distinct $\mathrm{CH}$ sequences $u$ and $v$ satisfy the following inequality:

$$
\forall k, l \in[0, T-1], \mathcal{C}(\text { rotate }(u, k), \operatorname{rotate}(v, l)) \geq m,
$$

where the positive integer $m$ is the degree of overlapping of the ACH system.

If the sequences of a sender and a receiver belong to an $\mathrm{ACH}$ system, the two nodes can rendezvous with each other on at least $m$ distinct rendezvous channels even if their clocks are asynchronous.

The following theorem states that a $\mathrm{CH}$ system with the rotation closure property ensures rendezvous even when the slot boundaries or $\mathrm{CH}$ sequence boundaries are not aligned.

Theorem 3. If a $\mathrm{CH}$ system $\mathrm{H}$ of period $\mathrm{T}$ with a degree of overlapping $m$ satisfies the rotation closure property, any pair of $\mathrm{CH}$ sequences in $H$ must overlap by at least $\mathrm{m} / 2$ timeslots for every $T$ consecutive timeslots even when the timeslot boundaries are misaligned by an arbitrary amount.

Proof. Suppose that a $\mathrm{CH}$ system $H$ satisfies the rotation closure property and two nodes, $A$ and $B$, each picks a $\mathrm{CH}$ sequence from $H$ randomly—viz, $u$ and $v$, respectively. For the sake of our discussions, suppose the length of a timeslot is 1 . We consider two cases. 
1) When slot boundaries are aligned: Without loss of generality, let us suppose node $A$ 's clock is $i$ slots ahead of node $B$ 's clock. With respect to node $B$ 's clock, node $A$ 's sequence $u$ is equivalent to $\operatorname{rotate}(u, i)$. Since $H$ has the rotation closure property, $C(\operatorname{rotate}(u, i), v) \geq m$. Hence, the two sequences must have at least $m$ rendezvous channels between them (i.e., overlap by at least $m$ timeslots). It is obvious that the same result is obtained when we assume that $A$ 's clock is $i$ slots behind $B$ 's clock.

2) When slot boundaries are misaligned: Suppose node $A$ 's clock is ahead of node $B$ 's clock by an arbitrary amount of time, say $i+\delta$, where $i \in \mathbf{Z}_{T}, 0<\delta<1$.

- If $\delta \leq 1 / 2$, let us shift left node $B$ 's sequence by $\delta$ and designate this sequence as $v^{\prime}{ }^{1}$ It is obvious that the slot boundaries of $u$ and $v^{\prime}$ are aligned and the former is ahead of the latter by $i$ slots in terms of their respective nodes' clocks. Since $H$ has the rotation closure property, $C\left(\right.$ rotate $\left.(u, i), v^{\prime}\right) \geq m$, and thus $u$ must overlap with $v$ by $m(1-\delta)$ timeslots. This means that the two sequences overlap with each other by at least $m / 2$ timeslots for every $T$ consecutive timeslots.

- If $\delta>1 / 2$, let us shift right node $B$ 's sequence by $1-\delta$ and designate this sequence as $v^{\prime}$. It is obvious that the slot boundaries of $u$ and $v^{\prime}$ are aligned and the former is ahead of the latter by $i+1$ slots in terms of their respective nodes' clocks. Since $H$ has the rotation closure property, $C\left(\right.$ rotate $\left.(u, i+1), v^{\prime}\right) \geq m$, and thus $u$ must overlap with $v$ by $m \delta$ timeslots. This means that the two sequences overlap with each other by at least $m / 2$ timeslots for every $T$ consecutive timeslots. $\delta$.

${ }^{1}$ Shifting a node's CH sequence left/right by $\delta$ is equivalent to advancing/retreating the node's clock by 
From Theorem 3, we can conclude that any two nodes that select $\mathrm{CH}$ sequences from a system with the rotation closure property can rendezvous with each other during the overlap of their sequences even if they are asynchronous (i.e., slot boundaries are not aligned). If multiple pairs of nodes happen to rendezvous at the same slot on the same channel, they can follow a channel contention procedure (e.g., 802.11 RTS/CTS protocol) to carry out the pairwise rendezvous.

The ACH System Design Problem. In the OSS paradigm, the appearance of primary user signals can hamper the secondary user nodes' rendezvous process, since primary users can occupy any given channel and have absolute priority over all channels. To minimize the chance of rendezvous failure due to those factors, we would ideally want an $\mathrm{ACH}$ system with the following property: in an OSS environment with $N$ available channels, an ACH system of period $T$ guarantees $N$ distinct rendezvous channels between any two $\mathrm{CH}$ sequences for every $T$ consecutive timeslots, i.e., its degree of overlapping is $m=N$.

Our research findings indicate that only certain types of quorum systems satisfy the rotation closure property and that only such quorum systems can guarantee pairwise rendezvous between two asynchronous sequences. An example is given below.

- Suppose we have a quorum system $Q$, under the universal set $U=\mathbf{Z}_{n}$, that satisfies the rotation closure property. Two quorums $p, q \in Q$ must have an intersection even if an arbitrary amount of cyclic rotation is applied to either one of them.

- Suppose we have an ACH system that includes two sequences of period $T=n$, i.e., $u$ and $v$. Given a channel index $h \in[0, N-1]$, we assign the channel index $h$ to $u$ and $v$ in the following way: let $u_{i}=h$ if $i \in p$ and let $v_{i}=h$ if $i \in q$, where $i \in[0, T-1]$. It is obvious that the rotation closure property of $Q$ guarantees that 
the two sequences rendezvous on channel $h$ even if an arbitrary amount of cyclic rotation is applied to either of the sequences.

\subsection{The ACH System with A Degree of Overlapping $m=$ 2}

In this section, we describe an asynchronous $\mathrm{CH}$ system that does not require global clock synchronization. The objective is to devise a $\mathrm{CH}$ system, $H$, that enables any pair of $\mathrm{CH}$ sequences to overlap by at least half of a timeslot for every sequence period (i.e., for every $T$ consecutive timeslots) even under the assumption that slot boundaries are misaligned by an arbitrary amount.

Next, we describe an algorithm, Algorithm 2, for constructing an $\mathrm{ACH}$ system that uses two different types of cyclic quorum systems. Note that the constructed ACH system has a degree of overlapping $m=2$, and this it is referred to as an asynchronous QCH $(\mathrm{AQCH})$ system.

\subsubsection{Construction of AQCH Systems}

Algorithm 2 uses two types of cyclic quorums systems to construct an AQCH system that guarantees at least two rendezvous channels between any two sequences (i.e., $C(u, v) \geq$ $2, \forall u, v \in H$ ). This AQCH system is composed of $\mathrm{CH}$ sequences that have only one frame per sequence period (i.e., $T=k$ ). In each constructed $\mathrm{CH}$ sequence, a subsequence constructed by a minimal cyclic quorum $S$ is interleaved with a subsequence constructed by a majority cyclic quorum system $S^{\prime}$. Refer to [42] for methods to construct minimal 


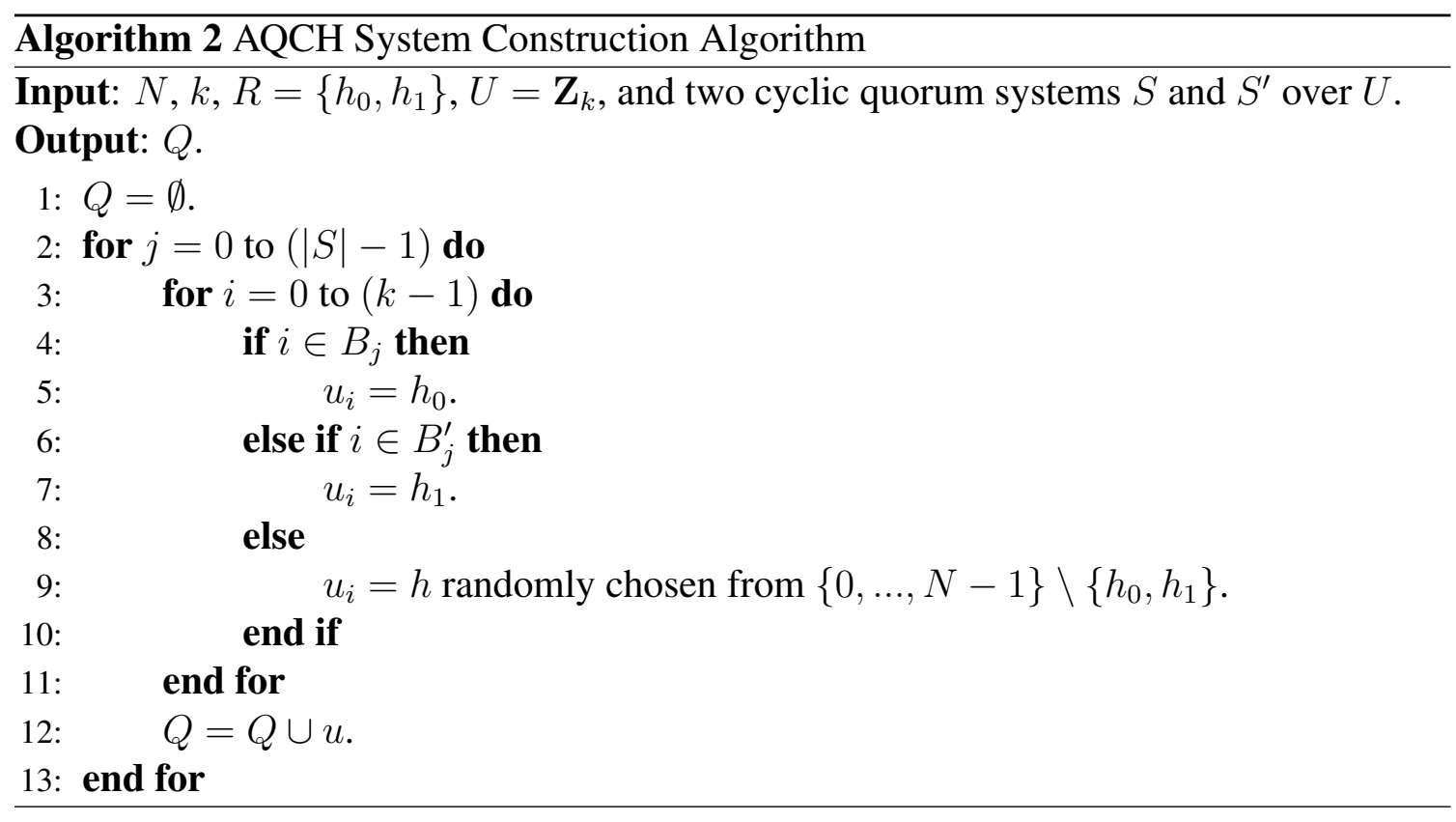

cyclic quorums and majority cyclic quorums. A pseudo-code of Algorithm 2 is given above and its description is given below.

1. First construct a universal set $U=\mathbf{Z}_{k}$;

2. Find a minimal $(k, \kappa)$-difference set $D=\left\{a_{1}, a_{2}, \ldots, a_{\kappa}\right\}$ such that $\kappa<\frac{k}{2}$ and construct a minimal cyclic quorum system based on $D$, such as $S=\left\{B_{i} \mid B_{i}=\right.$ $\left.\left\{a_{1}+i, a_{2}+i, \ldots, a_{\kappa}+i\right\} \bmod k, i \in[0, k-1]\right\}$;

3. Construct a relaxed cyclic $\left(k,\left\lceil\frac{k+1}{2}\right\rceil\right)$-difference set $D^{\prime}=\left\{a_{1}^{\prime}, a_{2}^{\prime}, \ldots, a_{\left\lceil\frac{k+1}{2}\right\rceil}^{\prime}\right\}$ such that $D^{\prime} \cap D=\emptyset$. Then, construct a majority cyclic quorum system based on $D^{\prime}$, such as $S^{\prime}=\left\{B_{i}^{\prime} \mid B_{i}^{\prime}=\left\{a_{1}^{\prime}+i, a_{2}^{\prime}+i, \ldots, a_{\left\lceil\frac{k+1}{2}\right\rceil}^{\prime}+i\right\} \bmod k, i \in[0, k-1]\right\}$. Note that $|S|=\left|S^{\prime}\right|=k$, and $\left|D^{\prime}\right|=\left\lceil\frac{k+1}{2}\right\rceil$;

4. Use the minimal cyclic quorum system $S$ for assigning the first rendezvous channel $h_{0}$ to appropriate slots in a $\mathrm{CH}$ sequence $u$ (see lines 4 and 5 in the algorithm); 
5. Use the majority cyclic quorum system $S^{\prime}$ to assign the second rendezvous channel $h_{1}$ to appropriate slots in a $\mathrm{CH}$ sequence $u$ (see lines 6 and 7 in the algorithm).

6. The remaining slots in $u$ are assigned a channel index randomly chosen from $\{0, \ldots, N-$ $1\} \backslash\left\{h_{0}, h_{1}\right\}$ (see line 9 in the algorithm).

An example ACH system is shown in Figure 4.1. One can readily show that an ACH system constructed using Algorithm 2 satisfies the rotation closure property and that the TTR value between any two sequences in an ACH system is bounded by the length of its sequence period $T=k$.

Given that $D^{\prime} \cap D=\emptyset, k$ must be no less than $|D|+\left|D^{\prime}\right|$ so that a $\mathrm{CH}$ sequence can accommodate two subsequences constructed using $S$ and $S^{\prime}$. In [42], Luk and Wong conducted an exhaustive search to find the minimal difference sets under $\mathbf{Z}_{k}$ for $k=$ $4, \ldots, 111$.

In our description of $\mathrm{ACH}$ systems given above, we used relaxed cyclic difference sets $D$ and $D^{\prime}$ for generating cyclic quorum systems that facilitate the construction of a $\mathrm{CH}$ system with the rotation closure property. The specific choices of $D$ and $D^{\prime}$ and the resulting cyclic quorum systems have no significance-i.e., the quorum systems that we have chosen are merely our design choices for constructing an ACH system; it is likely that there are other quorum systems that can be used to construct $\mathrm{ACH}$ systems of similar or different structure.

With two disjoint cyclic difference sets under $\mathbf{Z}_{T}$, one can readily construct two cyclic quorum systems. These quorum systems can be used to generate $\mathrm{CH}$ sequences of period $T$ that have two pairwise rendezvous channels, even in a time asynchronous environment. To construct an $\mathrm{ACH}$ system of period $T$ that enables $N$ rendezvous channels between 
$\mathrm{CH}$ sequences, we have to find $N$ disjoint cyclic difference sets under $\mathbf{Z}_{T}$. However, identifying $N$ disjoint cyclic difference sets under $\mathbf{Z}_{T}$ is a difficult problem in general. In fact, in many cases, $N$ disjoint cyclic difference sets under $\mathbf{Z}_{T}$ may not exist for some given values of $N$ and $T$. For example, the number of disjoint cyclic difference sets under $\mathbf{Z}_{12}$ when $N=3$ and $T=12$ is less than three. Thus, we will show how to construct an $\mathrm{ACH}$ with the maximum degree of overlapping using the second category of quorum systems, i.e., the array-based quorum system.

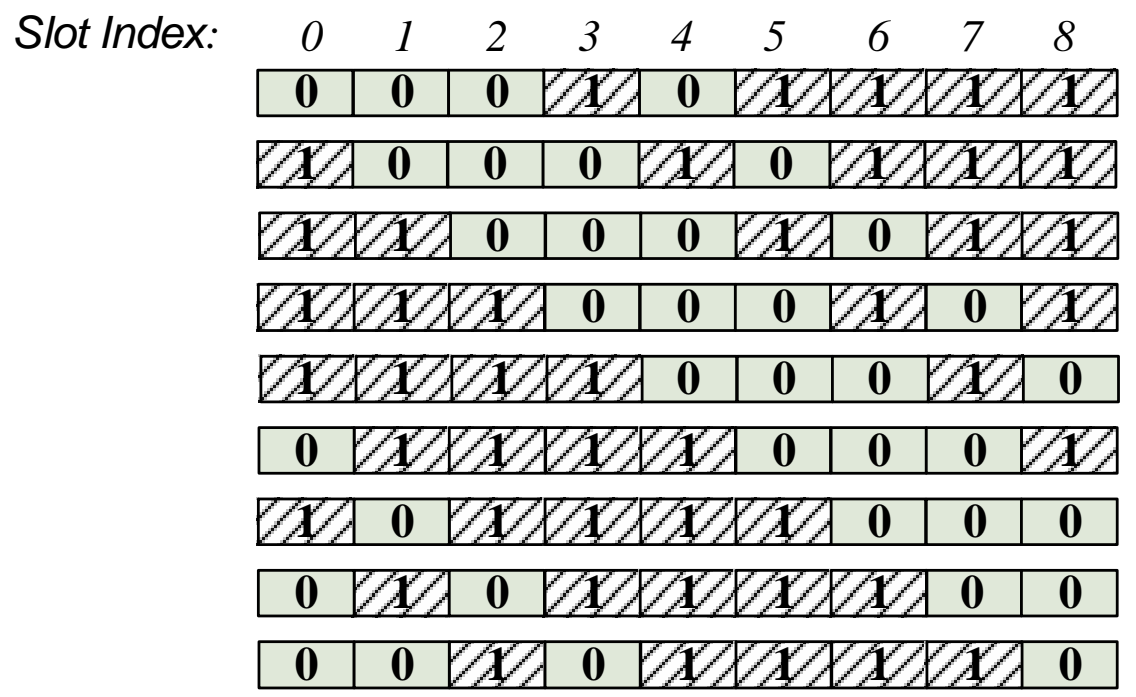

Figure 4.1: An ACH system $Q$ with $m=2$ and $T=k=9$. The universal set, $U$, is $\mathbf{Z}_{9}$, $S$ is constructed using $D=\{0,1,2,4\}$, and $S^{\prime}$ is constructed using $D^{\prime}=\{3,5,6,7,8\}$. Note that $D^{\prime} \cap D=\emptyset$. The numbers inside the slots represent the channel index values. 


\subsection{The ACH System with Maximum Degree of Overlap- ping}

\subsubsection{Array-based Quorum Systems}

In [36], the authors identified a few types of quorum systems that have the rotation closure property. We classify these quorum systems into two categories based on their construction methods. The first category of quorum systems is the cyclic quorum system that can be constructed using cyclic difference sets, which we have introduced in Chapter 2 . Then, we introduce the second category of quorum systems.

This category of quorum systems can be constructed using an $r \times l$ array, $A[\cdot][\cdot]$. The universal set $U$ is $\{0, \ldots, r \cdot l-1\}$, and there is a one-to-one mapping between the element $(i \cdot l+j)$ where $i \in[0, r-1], j \in[0, l-1]$ in the universal set and the array element $A[i][j]$.

- In the square quorum system [42], a quorum is constructed by picking a full column and a full row of elements from the array.

- In the torus quorum system [36], a quorum is constructed by picking a full column $c \in[0, l-1]$ plus $\lfloor l / 2\rfloor+1$ elements, each of which is located in distinct columns $(c+i) \bmod l, i=0, \ldots,\lfloor l / 2\rfloor$.

- We propose a new type of array-based quorum system that extends the torus quorum system. In this quorum system, a quorum is constructed by picking a full column of elements, $c \in[0, l-1]$, plus $l$ elements, each one located in a different column of 
the array. Since any quorum in this array-based quorum system is a super set of a torus quorum, the proposed array-based quorum system satisfies the rotation closure property.

As stated above, a quorum in an array-based quorum system must contain a full column of elements and $k$ elements $(k=l$ or $k=\lfloor l / 2\rfloor+1)$ selected from different columns of the array. We call the full column of elements as the column of the quorum and the other $k$ elements as the span of the quorum. Note that the column and the span of the same quorum have a common element.

An example of the array-based quorum system is illustrated in Figure 4.2. In the example, given two quorums, $p$ and $q$, the column of $p$ must have an intersection with the span of $q$, even if a "cyclic rotation" is applied to either $p$ or $q$, and vice versa. The cyclic rotation to $q$ will generate another quorum, $q^{\prime}$, of the same type. These properties hold for any two quorums in an array-based quorum system.

\subsubsection{Constructing ACH Systems with Maximum Degree of Overlap- ping}

In this subsection, we introduce an algorithm that uses an array-based quorum system to construct an $\mathrm{ACH}$ system that can be used by secondary nodes to establish links in a distributed manner. Suppose we want to construct an ACH system that enables any pair of $\mathrm{CH}$ sequences to rendezvous in $N$ distinct channels, where $N$ is the total number of available channels. The algorithm generates a set of $\mathrm{CH}$ sequences of period $T=r \cdot l$, given an $r \times l$ array where $r, l \geq N$. The reasons for utilizing an array-based quorum system are: 


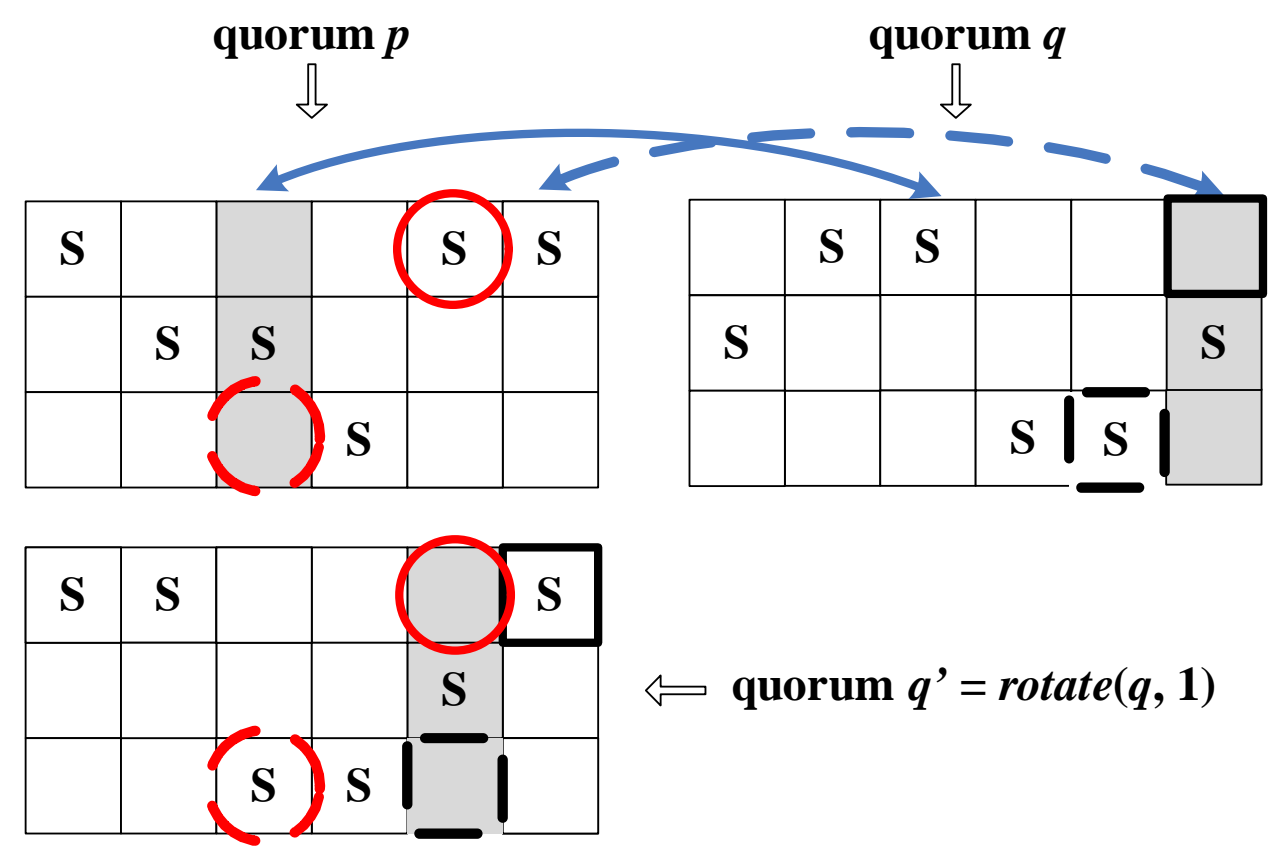

Figure 4.2: Two quorums, $p$ and $q$, each with 8 elements, in an array-based quorum system. Both quorums are constructed using a $3 \times 6$ array. An array-based quorum includes a column of elements (squares colored in grey) and a span of elements (squares labeled with a ' $\mathrm{S}$ '). A span and a column have one element in common which is not counted twice. One quorum's column must have an intersection with a span of another. For instance, in the figure, $p$ 's column intersects $q$ 's span at the square that is located in the first row of the third column. Similarly, $p$ 's span intersects $q$ 's column at the square located in the first row of the sixth column. Note that performing cyclic rotation by one slot to either of the array-based quorums yields a third array-based quorum (e.g., $\left.q^{\prime}=\operatorname{rotate}(q, 1)\right)$, and the intersection property is maintained after the cyclic rotation. The elements in common between $p$ and $q^{\prime}$ are marked with circle markings, and those between $q$ and $q^{\prime}$ are marked with square markings. 
- The intersections between the columns and the spans enables the pair of sequences (i.e., sender's and receiver's sequences) to rendezvous on every one of the $N$ channels;

- The rotation closure property of the array-based quorum system guarantees rendezvous on every one of the $N$ channels even if a cyclic rotation is applied to either of the two sequences.

The sender (or the receiver) generates an array-based quorum system using an $r \times l$ array, assigns channel index values to quorums' columns (or spans), and derives the $\mathrm{CH}$ sequence from the channel index assignment. The procedure for constructing the sequences is described below, and the corresponding pseudo code is given in Algorithm 3.

1. The sender constructs an $r \times l$ array, $S[\cdot][\cdot]$, which stores the channel index values assigned to columns' elements.

(a) The sender randomly assigns $N$ channel indexes to the $l$ columns of $S[\cdot \cdot[\cdot]$ such that each channel index is assigned to at least one column. All array elements in the same column are assigned the same channel index.

(b) Let $h_{i}$ denote the channel index assigned to the $i$-th column, where $i \in[0, l-1]$. The sender derives its $\mathrm{CH}$ sequence, $u$, in the following way: $u_{i \cdot l+j}=h_{j}$, where $i \in[0, r-1]$ and $j \in[0, l-1]$.

2. The receiver constructs an $r \times l$ array, $R[\cdot \cdot[\cdot]$, which stores the channel index values assigned to spans' elements. On basis of $R[\cdot][\cdot]$, an array-based quorum system can be constructed, from which the receiver chooses $r$ quorums that have $r$ disjoint 
spans. Let $s_{k}$ denote the span that contains the array element $R[k][0]$ where $k \in$ $[0, r-1]$ (see line 14 in Algorithm 3).

(a) The receiver randomly assigns $N$ channel indexes to the $r$ spans such that each channel index is assigned to at least one span, and all array elements belonging to the same span are assigned the same channel index.

(b) Let $h_{k}^{\prime}$ denote the channel index assigned to the span $s_{k}$. The receiver derives its $\mathrm{CH}$ sequence, $v$, in the following way: $v_{i \cdot l+j}=h_{k}^{\prime}$ if $R[i][j] \in s_{k}$ where $i \in[0, r-1]$ and $j \in[0, l-1]$.

Using Algorithm 3, we can assign the channel indexes to the sender's and receiver's $\mathrm{CH}$ sequences. We refer to $S[r][l]$ and $R[r][l]$ with their array elements fully assigned as the sequence array of the sender and the sequence array of the receiver, respectively. In the above method, channel indexes are assigned to array elements in a column-wise manner by the sender, or a span-wise manner by the receiver. Hence, we call the sender's $\mathrm{CH}$ sequence $u$ as a column-based sequence, and the receiver's $\mathrm{CH}$ sequence $v$ as a spanbased sequence. Figure 4.3 illustrates an example $\mathrm{ACH}$ system built using a $3 \times 5$ array when $N=3$. In the example, the degree of overlapping between the two sequences is $N$.

Theorem 4. Algorithm 3 generates an ACH system, $H=\{u, v\}$, such that the pair of CH sequences rendezvous in $N$ distinct channels within a sequence period, even if the two sequences are misaligned by an arbitrary amount.

Proof. For the sake of our discussions, suppose the length of a timeslot is 1 . We prove this theorem in two scenarios.

Case 1: Slot boundaries aligned. As previously mentioned, Algorithm 3 generates 


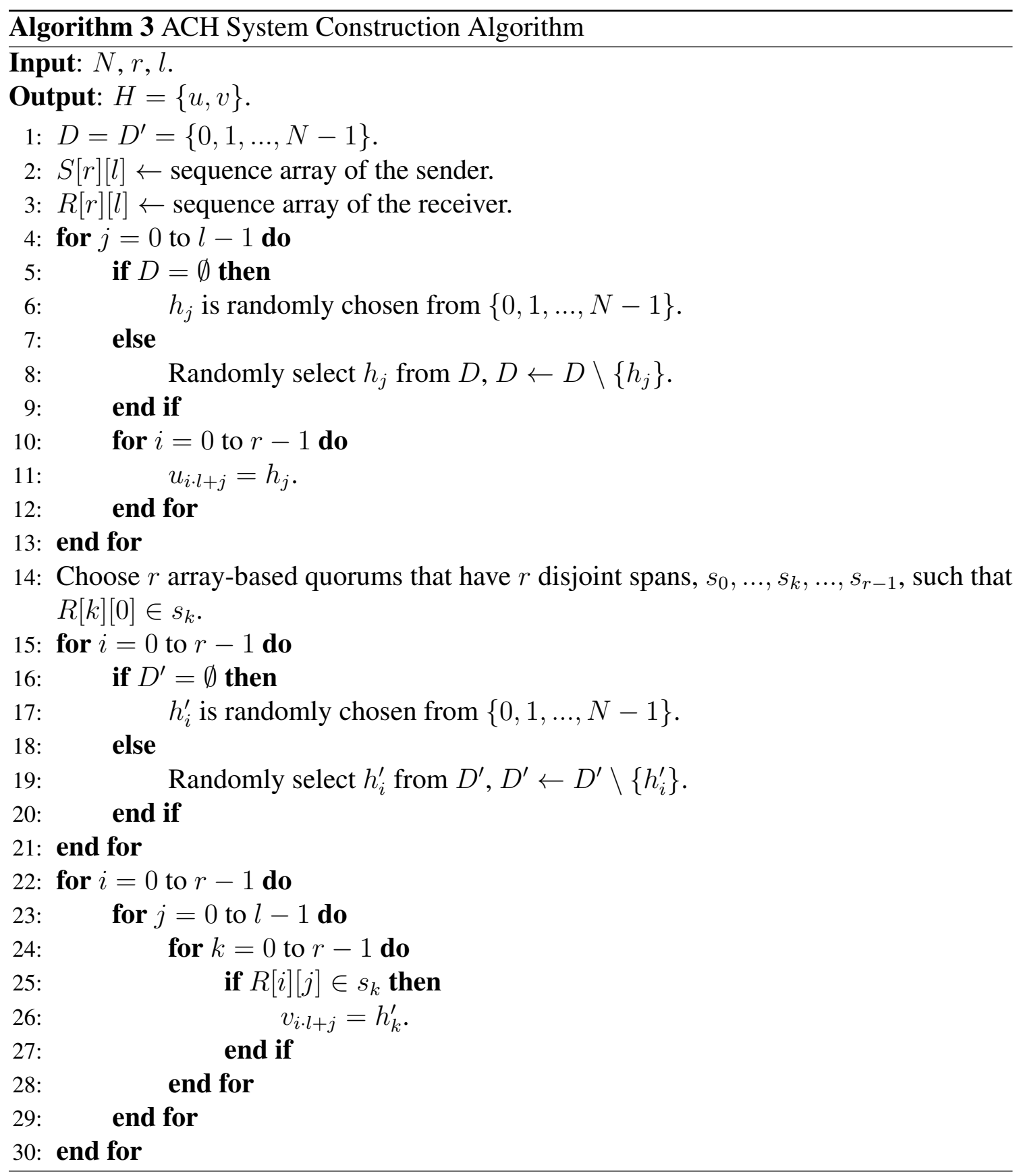




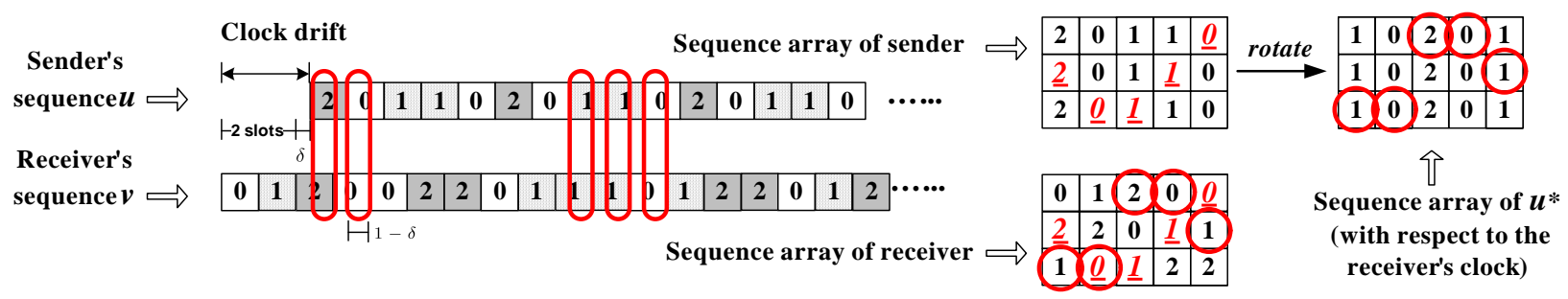

Figure 4.3: An ACH system $H$ when $N=3, r=3, l=5$, and the receiver's clock is ahead of the sender's clock by $(2+\delta)$ timeslots where $\delta \leq 1 / 2$. The sequence arrays of the sender and the receiver that generated the respective sequences are also shown. It can be easily seen that the two $\mathrm{CH}$ sequences have $N$ distinct rendezvous channels despite the clock drift and that the overlap duration on each rendezvous channel is $(1-\delta)$.

the sender's and receiver's CH sequences using two $r \times l$ arrays. Without loss of generality, let us suppose the receiver's clock is $i$ slots ahead of the sender's clock, where $i$ is an integer. Suppose that their timeslot boundaries are aligned. With respect to the receiver's clock, sender's sequence $u$ is equivalent to $\operatorname{rotate}(u, i)$, and the operation $\operatorname{rotate}(u, i)$ yields a new $\mathrm{CH}$ sequence $u^{*}$. In the sequence array of $u^{*}$, all the elements in each column are assigned the same channel index, and thus $l$ columns contain $N$ channel indexes.

If the sequence array of $u^{*}$ and the sequence array of the receiver (see the example in Figure 4.3) are superimposed on top of one another, every column of the former overlaps with one of the spans of the latter. Since $r, l \geq N$, one can readily observe that there are $N$ overlaps of $N$ distinct channel indexes. An overlap occurs when the same channel index appears in the same position (i.e., same row and column positions) in the two arrays. Thus, $u^{*}$ and $v$ have $N$ distinct rendezvous channels within a sequence period.

Case 2: Slot boundaries misaligned. In this case, suppose the receiver's clock is ahead of the sender's clock by an arbitrary amount of time, say $(i+\delta)$, where $0<\delta<1$.

- If $\delta>1 / 2$, let us shift right the sender's sequence by $1-\delta$ and designate this 
sequence as $u^{\prime}$. It is obvious that the timeslot boundaries of $u^{\prime}$ and $v$ are aligned and the latter is ahead of the former by $(i+1)$ slots in terms of their respective nodes' clocks. In Case 1 , we have shown that $C(\operatorname{rotate}(u, i), v)=N$ for any integer $i$. Since $\delta<1, u^{\prime}$ and $v$ rendezvous in $N$ distinct channels within a sequence period and the duration of overlap on each rendezvous channel is $\delta$.

- If $\delta \leq 1 / 2$ (the case shown in Figure 4.3), let us shift left the sender's sequence by $\delta$ and designate this sequence as $u^{\prime}$. It is obvious that the timeslot boundaries of $u^{\prime}$ and $v$ are aligned and the latter is ahead of the former by $i$ slots in terms of their respective nodes' clocks. Since $C(\operatorname{rotate}(u, i), v)=N$ for any integer $i$ and $\delta<1, u^{\prime}$ and $v$ rendezvous in $N$ distinct channels within a sequence period and the duration of overlap on each rendezvous channel is $(1-\delta)$.

By proving Theorem 4 we have validated that Algorithm 3 generates a pair of $\mathrm{CH}$ sequences that can be used by two channel hopping nodes to solve the asynchronous rendezvous problem in the context of opportunistic spectrum sharing. Note that the ability to rendezvous in $N$ distinct channels is a critical feature-it minimizes the chance of rendezvous failure due to appearance of primary user signals.

\subsection{Optimal ACH Systems}

In this section, we first introduce the metric for evaluating $\mathrm{CH}$ schemes that do not require clock synchronization-minimum rendezvous probability (MRP). Then, we formulate the optimal ACH design problem for CR networks. 


\subsubsection{Minimum Rendezvous Probability}

The minimum rendezvous probability (MRP) of an ACH system is defined as the lower bound of the probability that a pair of asynchronous sequences from an $\mathrm{ACH}$ system will rendezvous in a given timeslot. In an $\mathrm{ACH}$ system $H$ with period $T$ and a degree of overlapping value of $m$, two sequences are guaranteed to rendezvous at least $m$ times on $m$ distinct rendezvous channels during a sequence period. Thus, we define the MRP for an $\mathrm{ACH}$ system $H$ as,

$$
P(H)=\frac{m}{T}
$$

\subsubsection{The Optimal ACH Design Problem}

As stated previously, an ACH system that can serve as a solution to the asynchronous rendezvous problem is a system whose degree of overlapping is equal to the total number of available channels, viz $N$. The MRP of such a system is $\frac{N}{T}$, and maximizing the MRP for a fixed value of $N$ is equivalent to minimizing the sequence period, $T$. We define an optimal ACH system as a system whose degree of overlapping is $N$ and has the maximum possible MRP value. To devise an optimal ACH system, one has to solve the following optimization problem:

Problem 4. Given an ACH system $H=\{u, v\}$ of period $T$ and assuming there are $N$ available channels,

$$
\begin{aligned}
& \operatorname{minimize} \quad T, \\
& \text { subject to } \forall k, l \in[0, T-1], \\
& \qquad C \text { rotate }(u, k), \operatorname{rotate}(v, l))=N .
\end{aligned}
$$


The solution to Problem 4 is given by the following theorem.

Theorem 5. In an ACH system $H$ whose degree of overlapping is $N$, the period of any CH sequence must be $N^{2}$ or greater.

Proof. Suppose we have two sequences, $u$ and $v$, from an $\mathrm{ACH}$ system of period $T$. Let $k_{u, h}$ denote the number of timeslots in sequence $u$ that are assigned with the channel index $h \in[0, N-1]$. Then, we can express the period length $T$ as

$$
T=\sum_{h=0}^{N-1} k_{u, h}=\sum_{h=0}^{N-1} k_{v, h}=\sum_{h=0}^{N-1}\left(\frac{k_{u, h}+k_{v, h}}{2}\right) .
$$

Without loss of generality, we fix $u$ and cyclically rotate $v$ by $l, l=0,1, \ldots, T-1$. Let us count the total accumulated number of rendezvous between $u$ and rotate $(v, l)$ as $l$ is incremented from 0 to $T-1$. Since $\mathcal{C}(u, \operatorname{rotate}(v, l))=N$, for any channel $h \in[0, N-1]$, the total number of rendezvous that involve a given timeslot, $v_{i}=h$, in $v$ is $k_{u, h}$. Since there are $k_{v, h}$ timeslots in $v$ that are assigned channel $h$, the total accumulated number of rendezvous between $u$ and $\operatorname{rotate}(v, l)$, as $l$ is incremented from 0 to $T-1$, in which the rendezvous channel is $h$ is $k_{u, h} \cdot k_{v, h}$.

By definition, $\mathcal{C}(u, \operatorname{rotate}(v, l))=N$ for any amount of cyclic rotation to $v$. This means that $u$ and rotate $(v, l)$ must rendezvous in channel $h$ at least once. Hence, the total accumulated number of rendezvous in channel $h$ (as $l$ is incremented from 0 to $T-1$ ) is at least $T$. Thus, we have $k_{u, h} \cdot k_{v, h} \geq T$.

Since $\left(\frac{k_{u, h}+k_{v, h}}{2}\right)^{2} \geq k_{u, h} \cdot k_{v, h}$, we can readily derive

$$
\frac{k_{u, h}+k_{v, h}}{2} \geq \sqrt{T}
$$

Combining (4.2) and (4.3), we get

$$
T=\sum_{h=0}^{N-1}\left(\frac{k_{u, h}+k_{v, h}}{2}\right) \geq N \sqrt{T} .
$$


Therefore, we conclude that $T \geq N^{2}$.

In the next two sections, we describe two optimal $\mathrm{ACH}$ designs in which the period of the $\mathrm{CH}$ sequences is $O\left(N^{2}\right)$.

\subsection{Asymmetric Optimal ACH Systems}

Optimal ACH designs can be categorized into two types: the asymmetric design and the symmetric design that both achieve maximum rendezvous diversity (i.e., maximum number of rendezvous channels) with a minimum period of a $\mathrm{CH}$ sequence.

An asymmetric design of the optimal $\mathrm{ACH}$ system can be constructed using Algorithm 3 with an $N \times N$ array-based quorum system. A pair of sequences in an optimal $\mathrm{ACH}$ system has $N$ pairwise rendezvous channels and the sequence period is $N^{2}$ timeslots. Thus, an optimal ACH system has an MRP value of $1 / N$. We refer to $S$ and $R$ used in the construction algorithm as the sequence array of the sender and the sequence array of the receiver, respectively. In the above methods, channel indexes are assigned to array elements in a column-wise manner by the sender, or a span-wise manner by the receiver. Hence, we call the sender's CH sequence $u$ as a column-based sequence, and the receiver's $\mathrm{CH}$ sequence $v$ as a span-based sequence. Figure 4.4 illustrates an example $\mathrm{ACH}$ system built using a $3 \times 3$ array when $N=3$ and $u^{*}$ defines the sender's $\mathrm{CH}$ sequence after a cyclic rotation by $(2+\delta)$. 


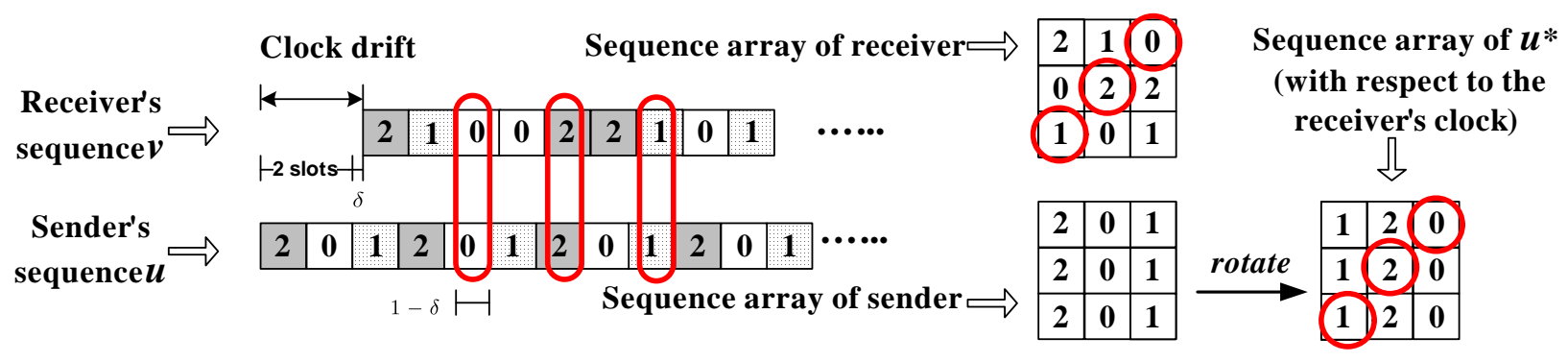

Figure 4.4: An asymmetric design of an optimal ACH system when $N=3$. The sender's clock is ahead of the receiver's clock by $(2+\delta)$ timeslots where $\delta \leq 1 / 2$. The sequence arrays of the sender and the receiver that generated the respective $\mathrm{CH}$ sequences are shown. It can be easily seen that the two $\mathrm{CH}$ sequences have $N$ distinct rendezvous channels despite the clock drift and that the overlap duration in each rendezvous channel is $(1-\delta)$.

\subsection{Symmetric Optimal ACH Systems}

\subsubsection{Limitations of Asymmetric ACH Systems}

An ACH system created using the asymmetric design approach requires each radio to have a pre-assigned role as either a sender or a receiver. Although such a requirement may be acceptable in some scenarios (e.g., half-duplex communication systems or in Bluetooth pairing), it may not be acceptable in systems where a radio's role as sender/receiver is not pre-assigned. Designing time-asynchronous rendezvous protocols that are symmetric is a challenging problem. In the next subsection, we will explain how to devise symmetric ACH systems.

\subsubsection{Construction of Symmetric ACH Systems}

To construct a symmetric $\mathrm{ACH}$ system, we require every node to generate its $\mathrm{CH}$ sequence from a bit sequence that it possesses. Specifically, based on certain bit sequence design 


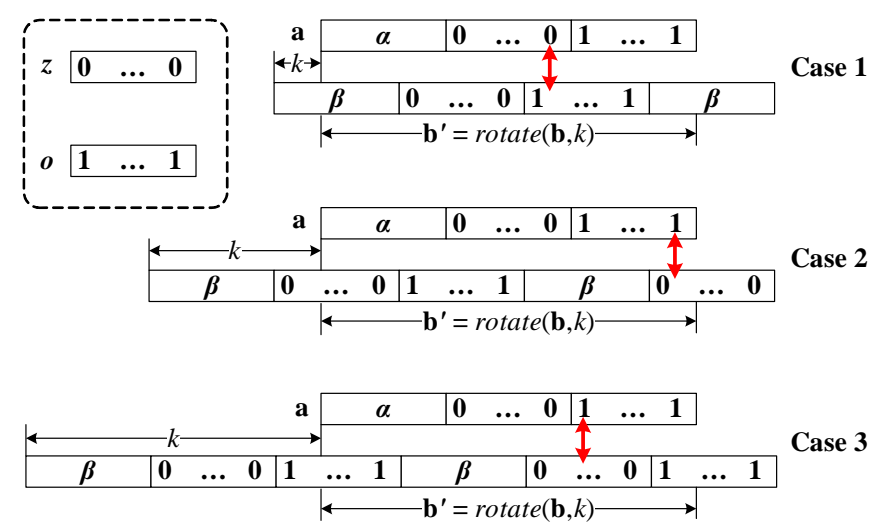

Figure 4.5: Illustration of the three cases Lemma 1's proof

techniques, two channel hopping nodes are able to construct two bit sequences that have at least one distinct bit. Then, every node replaces any bit of " 1 " (or " 0 ”) in its bit sequence with a column-based (or span-based) sequence. The resulting $\mathrm{CH}$ sequences of the two nodes would achieve the maximum rendezvous diversity owing to the maximum number of rendezvous channels guaranteed between a column-based sequence and a span-based sequence. Note that every node follows the same method of constructing its $\mathrm{CH}$ sequence, which belongs to a symmetric ACH system.

We define a bit sequence as a sequence of bits where each bit takes either a value of 0 or 1 . Note that a bit sequence is a special case of a $\mathrm{CH}$ sequence such that the channel indexes are chosen from $\{0,1\}$. Hence, the cyclic rotation operation is also applicable to a bit sequence. Let us introduce the bit sequence design problem which is defined as follows: Enable two nodes to independently construct two distinct bit sequences. Note that the sequences resulting from cyclic rotations of a sequence are not considered to be distinct with respect to each other and the original sequence.

Assume that a unique $n$-bit sequence is assigned to each network node and that $n$ is a system parameter. For example, the unique 48-bit $(n=48)$ sequence of a node can be the 
MAC address of the node's network interface. We refer to such a bit sequence as a node's ID sequence, and let $\alpha$ denote the $n$-bit ID sequence of node $x$. Now, let us define an $n$-bit sequence that contains zeros only:

$$
z=\left\{z_{0}, \ldots, z_{n-1}\right\}, \forall i \in[0, n-1], z_{i}=0
$$

and an $n$-bit sequence that contains ones only

$$
o=\left\{o_{0}, \ldots, o_{n-1}\right\}, \forall i \in[0, n-1], o_{i}=1
$$

Let $\|$ denote a concatenation operator that concatenates two bit or $\mathrm{CH}$ sequences. We define the expanded ID sequence of node $x$ as the concatenation of three $n$-bit sequences$\alpha, z$ and $o$-as given in the following equation:

$$
\mathbf{a}=\left\{a_{0}, \ldots, a_{3 n-1}\right\}=\alpha\|z\| o
$$

According to the following lemma, two expanded ID sequences generated from two different ID sequences are guaranteed to be distinct.

Lemma 1. Given any two $n$-bit sequences $\alpha=\left\{\alpha_{0}, \ldots, \alpha_{n-1}\right\}$ and $\beta=\left\{\beta_{0}, \ldots, \beta_{n-1}\right\}$, let $\mathbf{a}=\alpha\|z\| o$ and $\mathbf{b}=\beta\|z\|$, where $z$ is an $n$-bit sequence composed of only zeros and o is an n-bit sequence composed of only ones.

$$
\text { If } \alpha \neq \beta \text {, then }
$$

$$
\mathbf{a} \neq \operatorname{rotate}(\mathbf{b}, k), \forall k \in(0,3 n-1] \text {. }
$$

Proof. We prove the above statement by considering three possible scenarios, and showing, in each scenario, that a bit in $\mathbf{a}$ and another bit in $\operatorname{rotate}(\mathbf{b}, k)$ have different values although the two bits are in the same position within the respective expanded ID sequences. This is sufficient to prove that the two expanded ID sequences are not equal. Let $\mathbf{b}^{\prime}=\operatorname{rotate}(\mathbf{b}, k)$. 


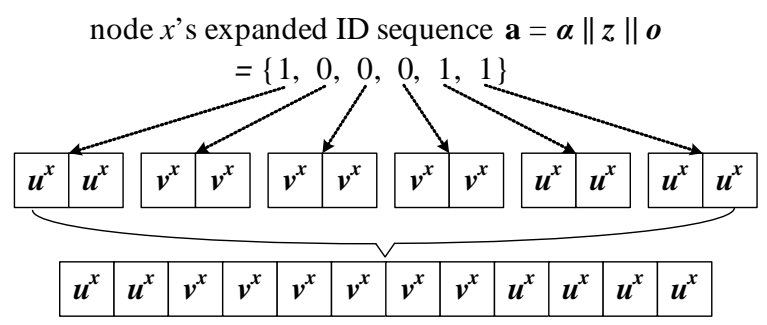

Figure 4.6: Construction of a $\mathrm{CH}$ sequence for the symmetric ACH scheme. In the above figure, node $x$ 's ID sequence is $\alpha=\{1,0\}, n=2, z=\{0,0\}$ and $o=\{1,1\}$. Node $x$ 's column- and span-based sequences are $u^{x}$ and $v^{x}$, respectively.

Case 1: $k \in(0, n]$. Since $\mathbf{a}=\alpha\|z\| o$ and $\mathbf{b}=\beta\|z\| o, a_{2 n-1}=0$ and $b_{2 n-1}^{\prime}=1$ (as indicated by the arrow in the Case 1 illustration of Figure 4.5).

Case 2: $k \in(n, 2 n]$. Since $\mathbf{a}=\alpha\|z\| o$ and $\mathbf{b}=\beta\|z\| o, a_{3 n-1}=1$ and $b_{3 n-1}^{\prime}=0$ (as indicated by the arrow in the Case 2 illustration of Figure 4.5).

Case 3: $k \in(2 n, 3 n-1]$. Since $\mathbf{a}=\alpha\|z\| o$ and $\mathbf{b}=\beta\|z\| o, a_{2 n}=1$ and $b_{2 n}^{\prime}=0$ (as indicated by the arrow in the Case 3 illustration of Figure 4.5).

Thus, we conclude that $\mathbf{a} \neq \operatorname{rotate}(\mathbf{b}, k), \forall k \in(0,3 n-1]$.

We now describe how to generate the $\mathrm{CH}$ sequences of a symmetric $\mathrm{ACH}$ system. Suppose that the number of available channels is $N$.

1. Suppose node $x$ has a unique ID sequence, $\alpha$, that contains $n$ bits, and its expanded ID sequence is $\mathbf{a}=\alpha\|z\| o$, which has $3 n$ bits. Lemma 1, ensures that two nodes that have two distinct ID sequences must have two distinct expanded ID sequences.

2. Using the procedure outlined in Section ?? for constructing asymmetric ACH systems, node $x$ that needs to establish rendezvous with neighboring nodes generates its own asymmetric $\mathrm{ACH}$ system (including a column-based sequence $u^{x}$ and a spanbased sequence $v^{x}$ ) independently of other nodes. Note that different nodes may 
generate different asymmetric ACH systems.

3. Node $x$ constructs its $\mathrm{CH}$ sequence by expanding each bit in a to a frame, which is mapped to a certain sequence as described below. From $i=0$ to $i=3 n-1$, each bit of a is expanded in the following way:

(a) if $a_{i}=1$, then the $(i+1)^{t h}$ frame of node $x$ 's $\mathrm{CH}$ sequence is the sequence $\left(u^{x} \| u^{x}\right)$

(b) if $a_{i}=0$, then the $(i+1)^{t h}$ frame of node $x$ 's $\mathrm{CH}$ sequence is the sequence $\left(v^{x} \| v^{x}\right)$.

Each frame is either a sequence $\left(u^{x} \| u^{x}\right)$ or $\left(v^{x} \| v^{x}\right)$, and thus it includes $2 N^{2}$ slots.

A simple example of the $\mathrm{CH}$ sequence construction method is illustrated in Figure 4.6. Every node has a distinct expanded ID sequence. In other words, every pair of distinct expanded ID sequences differ by at least one bit. For the sake of this discussion, let us say that they differ by a single bit at the $i$-th bit position. This implies that after the two expanded ID sequences are expanded, the $i$-th frame in each of the two resulting $\mathrm{CH}$ sequences will be composed of different types of sequences-viz, one frame is composed of a column-based sequence while the other frame is composed of a span-based sequence. As stated previously, a column-based sequence and a span-based sequence always overlap in $N$ distinct channels. Hence, any pair of nodes that construct $\mathrm{CH}$ sequences using the procedure described above would be able to rendezvous in the maximum number of distinct channels, which is $N$. To illustrate this point further, an example is given below.

Suppose node $x$ generates a column-based sequence $u^{x}$ and a span-based sequence $v^{x}$. Likewise, node $y$ generates $u^{y}$ and $v^{y}$. Moreover, suppose nodes $x$ and $y$ have expanded 
ID sequences 100011 and 000011, respectively. Following the aforementioned procedure, the two nodes generate their $\mathrm{CH}$ sequences respectively as

$$
\left(u^{x} \| u^{x}\right)\left\|\left(v^{x} \| v^{x}\right)\right\|\left(v^{x} \| v^{x}\right)\left\|\left(v^{x} \| v^{x}\right)\right\|\left(u^{x} \| u^{x}\right) \|\left(u^{x} \| u^{x}\right)
$$

and

$$
\left(v^{y} \| v^{y}\right)\left\|\left(v^{y} \| v^{y}\right)\right\|\left(v^{y} \| v^{y}\right)\left\|\left(v^{y} \| v^{y}\right)\right\|\left(u^{y} \| u^{y}\right) \|\left(u^{y} \| u^{y}\right) .
$$

The two nodes' expanded ID sequences are different in the first bit position, and the first frame of each node's $\mathrm{CH}$ sequence is $\left(u^{x} \| u^{x}\right)$ and $\left(v^{y} \| v^{y}\right)$ respectively. Since the first frame of one $\mathrm{CH}$ sequence is composed of two concatenated column-based sequences and the other is composed of two concatenated span-based sequences, nodes $x$ and $y$ are able rendezvous in $N$ distinct channels during the first half frame.

Note that node $x$ uses two concatenated column-based sequences, $\left(u^{x} \| u^{x}\right)$, to expand a bit with a value of one or two concatenated span-based sequences, $\left(v^{x}|| v^{x}\right)$, to expand a bit with a value of zero, instead of using a single column- or span-based sequence. This method is employed to guarantee that any pair of nodes that generate their $\mathrm{CH}$ sequences using the above procedure can rendezvous in $N$ channels irrelevant of the clock drift between them. For instance, if an expanded ID sequence's bit is expanded to a single column (or span) based sequence, then rendezvous in $N$ channels cannot be guaranteed when the clock drift is greater than zero and less than the length of a frame. An example is given in Figure 4.7 .

Algorithm 4 describes the procedure for generating $\mathrm{CH}$ sequences of a symmetric $\mathrm{ACH}$ system. The following theorem describes the properties of the $\mathrm{CH}$ sequences generated using Algorithm 4. 

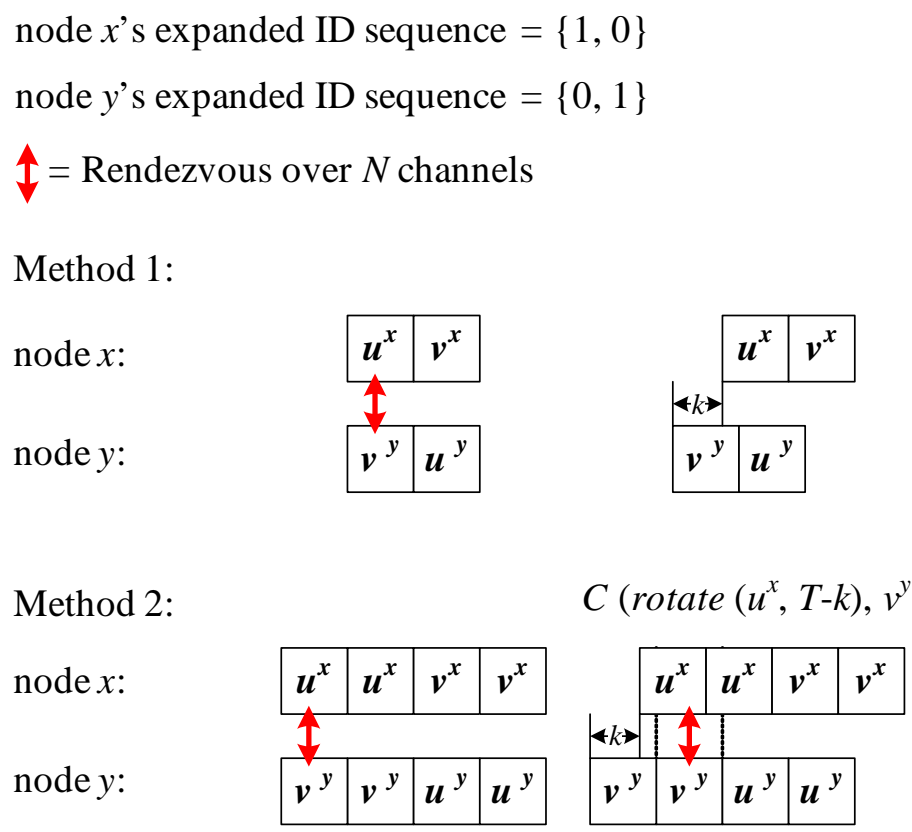

Figure 4.7: Motivation for using two concatenated column/span-based sequences instead of a single sequence. Suppose node $x$ generates column and span-based sequences $u^{x}$ and $v^{x}$; similarly, node $y$ generates sequences $u^{y}$ and $v^{y}$. Using two concatenated column or span-based sequences in the $\mathrm{CH}$ sequence construction method (method 2 in the figure), the maximum rendezvous diversity is guaranteed when two channel hopping nodes have a clock drift that is less than a frame length. In the example, given $N$ channels and a $\mathrm{CH}$ period of $T$, rotate $\left(u^{x}, T-k\right)$ yields another column-based sequence for node $x$, which overlaps with node $y$ ' span-based sequence $v^{y}$ over $N$ channels. On the contrary, the method of using a single column or span-based sequence (method 1 in the figure) is unable to guarantee such maximum rendezvous diversity, e.g., $u^{x}$ may not have $N$ rendezvous channels with a sequence concatenated by parts of $v^{y}$ and $u^{y}$.

Theorem 6. Algorithm 4 generates sequences of an ACH system whose period is $6 n N^{2}$ and degree of overlapping is $N$. Here, $N$ is the total number of channels, and $n$ is the length of a radio's ID sequence.

Proof. Suppose two arbitrary channel-hopping nodes, $x$ and $y$, have $n$-bit ID sequences $\alpha$ and $\beta$, respectively. Let $\mathbf{a}$ and $\mathbf{b}$ be the expanded ID sequences of nodes $x$ and $y$. Each node generates a column-based sequence and a span-based sequence independently of the 


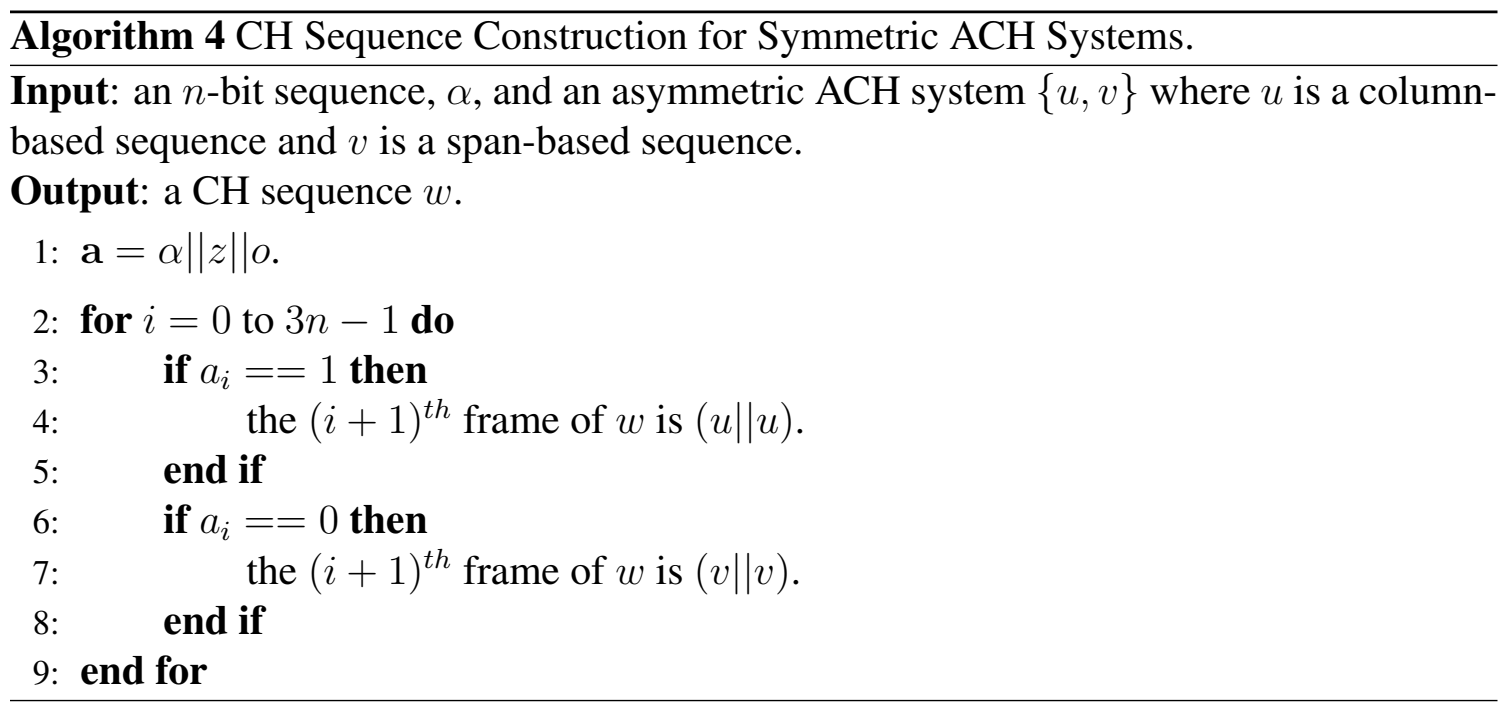

other node. Suppose node $x$ generates a column-based sequence $u^{x}$ and a span-based sequence $v^{x}$. Similarly, node $y$ generates a column-based sequence $u^{y}$ and a span-based sequence $v^{y}$. Using its $n$-bit ID sequence, $\alpha$, and the column-based sequence $u^{x}$ and the span-based sequence $v^{x}$ as inputs to Algorithm 4, node $x$ generates its $\mathrm{CH}$ sequence $w$. Similarly, using its $n$-bit ID sequence, $\beta, u^{y}$ and $v^{y}$ as inputs to Algorithm 4 , node $y$ generates its $\mathrm{CH}$ sequence $w^{\prime}$.

Since each $\mathrm{CH}$ sequence generated by Algorithm 4 (either $w$ or $w^{\prime}$ ) contains $3 n$ frames and each frame has exactly $2 N^{2}$ slots, the period of a $\mathrm{CH}$ sequence generated by the algorithm is $6 n N^{2}$.

Without loss of generality, suppose that node $x$ 's clock is $i$ slots ahead of node $y$ 's clock, where $i$ is an arbitrary integer. We prove that the $\mathrm{CH}$ sequences $\left\{w, w^{\prime}\right\}$ form an $\mathrm{ACH}$ system by considering the following two cases.

Case 1: $2 k N^{2}<i \leq(2 k+1) N^{2}$, where $k \in[0,3 n-1]$. In this case, node $x$ 's $\mathrm{CH}$ sequence is ahead of node $y$ 's $\mathrm{CH}$ sequence by $k$ frames and $\left(i-2 k N^{2}\right)$ slots. With respect to node $y$ 's clock, node $x$ 's $\mathrm{CH}$ sequence is equivalent to a $\mathrm{CH}$ sequence generated from 
the bit sequence $\operatorname{rotate}(\mathbf{a}, \kappa)$. Let $\mathbf{a}^{\prime}=\operatorname{rotate}(\mathbf{a}, \kappa)$, where $\kappa=i-2 k N^{2}$. According to Lemma 1 , we have $\operatorname{rotate}(\mathbf{a}, \kappa) \neq \mathbf{b}$. If $a_{j}^{\prime} \neq b_{j}$ for some $j \in[0,3 n-1]$, there are two possible cases to consider:

1. If $b_{j}=0$, then $a_{j}^{\prime}=1$. This implies that the $(j+1)$-th frame of $w^{\prime}$ is $\left(v^{y} \| v^{y}\right)$ and the $((j+\kappa+1) \bmod 3 n)$-th frame of $w$ is $\left(u^{x} \| u^{x}\right)$.

2. If $b_{j}=1$, then $a_{j}^{\prime}=0$. This implies that the $(j+1)$-th frame of $w^{\prime}$ is $\left(u^{y} \| u^{y}\right)$ and the $((j+\kappa+1) \bmod 3 n)$-th frame of $w$ is $\left(v^{x} \| v^{x}\right)$.

Case 2: $(2 k+1) N^{2}<i \leq(2 k+2) N^{2}$, where $k \in[0,3 n-1]$. In this case, node $x$ 's $\mathrm{CH}$ sequence is ahead of node $y$ 's $\mathrm{CH}$ sequence by an amount of $(k+1)$ frames and $\left(i-(2 k+2) N^{2}\right)$ slots. With respect to node $y$ 's clock, node $x$ 's $\mathrm{CH}$ sequence is equivalent to a $\mathrm{CH}$ sequence generated from a bit sequence rotate $(\mathbf{a}, \kappa+1)$. Let $\mathbf{a}^{\prime}=\operatorname{rotate}(\mathbf{a}, k+1)$ and $\kappa=(2 k+2) N^{2}-i$. According to Lemma 1 , we have rotate $(\mathbf{a}, \kappa+1) \neq \mathbf{b}$. If $a_{j}^{\prime} \neq b_{j}$ for some $j \in[0,3 n-1]$, there are also two possible cases to consider:

1. If $b_{j}=0$, then $a_{j}^{\prime}=1$. This implies that the $(j+1)$-th frame of the CH sequence $w^{\prime}$ is $\left(v^{y} \| v^{y}\right)$, the $((j+\kappa+2) \bmod 3 n)$-th frame of $\mathrm{CH}$ sequence $w$ is $\left(u^{x} \| u^{x}\right)$.

2. If $b_{j}=1$, then $a_{j}^{\prime}=0$. This implies that the $(j+1)$-th frame of the $\mathrm{CH}$ sequence $w^{\prime}$ is $\left(u^{y} \| u^{y}\right)$, the $((j+k+2) \bmod 3 n)$-th frame of $\mathrm{CH}$ sequence $w$ is $\left(v^{x} \| v^{x}\right)$.

In both cases, according to Theorem 2, a pair of sequences $\left(v^{y} \| v^{y}\right)$ and $\left(u^{x} \| u^{x}\right)$ (or $\left(u^{y} \| u^{y}\right)$ and $\left.\left(v^{x} \| v^{x}\right)\right)$ form an ACH system that has a degree of overlapping of $N$. This means that the pair of sequences have $N$ distinct rendezvous channels irrelevant of the $\kappa$ slot clock drift when $0 \leq \kappa \leq N^{2}$. Thus, $w$ and $w^{\prime}$ have $N$ distinct rendezvous channels. 
Since $i$ is an arbitrary value, we can conclude that $\forall k, l \in[0, T-1]$,

$$
\mathcal{C}\left(\text { rotate }(w, k), \text { rotate }\left(w^{\prime}, l\right)\right)=N
$$

and $\left\{w, w^{\prime}\right\}$ is an $\mathrm{ACH}$ system whose degree of overlapping is $N$.

According to Theorem 6, each node can rendezvous with another node without clock synchronization by independently generating $\mathrm{ACH}$ sequences using Algorithm 4. These $\mathrm{ACH}$ sequences enable maximum rendezvous diversity (i.e., rendezvous in $N$ distinct channels within a period of $6 n N^{2}$ ). Hence, the resulting ACH system's MRP value is $1 / 6 n N$, and its ATTR is $O(N)$.

Note that no two distinct nodes will generate the same $\mathrm{CH}$ sequence since their expanded ID sequences are different. Using Algorithm 4, any two nodes can achieve asynchronous rendezvous without being pre-assigned a sender/receiver role.

\subsection{Comparisons of ACH Schemes}

In this section, we compare the proposed optimal $\mathrm{ACH}$ designs with existing $\mathrm{CH}$ schemes that also support the asynchronous operation. We assume that $N$ is the total number of available channels.

Random channel hopping (RCH) [54]. In this scheme, each node hops from one channel to another randomly at the beginning of every timeslot. The $\mathrm{RCH}$ scheme has an ATTR value of $N$. However, the RCH scheme does not guarantee a bounded TTR between any two sequences, and its MRP value cannot be calculated either.

Sequence-based rendezvous (SR) [19]. Every node using SR follows the same predetermined $\mathrm{CH}$ sequence. The sequence period is $N(N+1)$, the degree of overlapping is 
Table 4.1: A comparison of asynchronous $\mathrm{CH}$ schemes.

\begin{tabular}{|c||c|c|c|}
\hline & $\begin{array}{c}\text { Degree } \\
\text { of } \\
\text { overlapping }\end{array}$ & MRP & ATTR \\
\hline \hline RCH & 0 & n/a & $N$ \\
\hline SR & 1 & $\frac{1}{N(N+1)}$ & $O\left(N^{2}\right)$ \\
\hline Asym. opt. ACH & $N$ & $\frac{1}{N}$ & $O(N)$ \\
\hline Sym. opt. ACH & $N$ & $\frac{1}{6 n N}$ & $O(N)$ \\
\hline
\end{tabular}

1 , and thus the MRP is $\frac{1}{N(N+1)}$.

A comparison of all the aforementioned schemes is summarized in Table 4.1. From the table, we can see that the proposed asymmetric and symmetric $\mathrm{ACH}$ schemes are superior to the other schemes in terms of degree of overlapping. Both schemes' degree of overlapping is the maximum possible value of $N$, and therefore the two schemes are optimal in terms of rendezvous diversity. On the other hand, the two schemes' ATTR is $O(N)$, which indicates that the ATTR scales linearly with the number of channels. The proposed optimal ACH systems and the SR scheme guarantee a bounded TTR between any two channel hopping nodes, while the $\mathrm{RCH}$ scheme cannot give an upper bounded TTR.

\subsection{Performance Evaluation}

\subsubsection{Simulation Setup}

In this section, we compare the performance of three symmetric $\mathrm{CH}$ schemes that do not require clock synchronization, namely $\mathrm{RCH}, \mathrm{SR}$, and the symmetric optimal $\mathrm{ACH}$, via simulation results. We simulate ten pairs of secondary nodes using ns-2 [63] in a 
$1000 \mathrm{~m} \times 1000 \mathrm{~m}$ square area. Every secondary node has a single half-duplex radio, and the transmission range of every secondary radio is $250 \mathrm{~m}$. In the simulations, the radios of all the secondary users and the primary users can access 11 channels (i.e., the number of channels available to the $\mathrm{CR}$ network is $N=11$ ). The duration of a timeslot is $10 \mathrm{~ms}$. Each secondary node independently generates its $\mathrm{CH}$ sequence using the agreed $\mathrm{CH}$ scheme and performs channel hopping in accordance with the sequence. Once two nodes rendezvous on a channel, the link between them is established. Ten independent simulation runs were conducted for each simulation result.

Primary user (PU) traffic generation. We simulated $X$ primary transmitters operating on $X$ channels, and those channels were randomly chosen in each simulation run. In most existing work, it is assumed that a primary transmitter follows a "busy/idle" transmission pattern on a licensed channel [28], and we make the same assumption. The busy period has a fixed length of $b$ timeslots, and the idle period follows an exponential distribution with a mean of $l$ timeslots. All of the secondary nodes are within the transmission range of any primary transmitter. A channel is considered "unavailable" when primary user signals are present in it. All secondary nodes should refrain from transmitting in unavailable channels.

Random clock drift. The simulations were performed under the assumption that the nodes' clocks are not synchronized. In each simulation run, each node determines its clock time independently of other nodes. Thus, there is a random clock drift between any two nodes. 


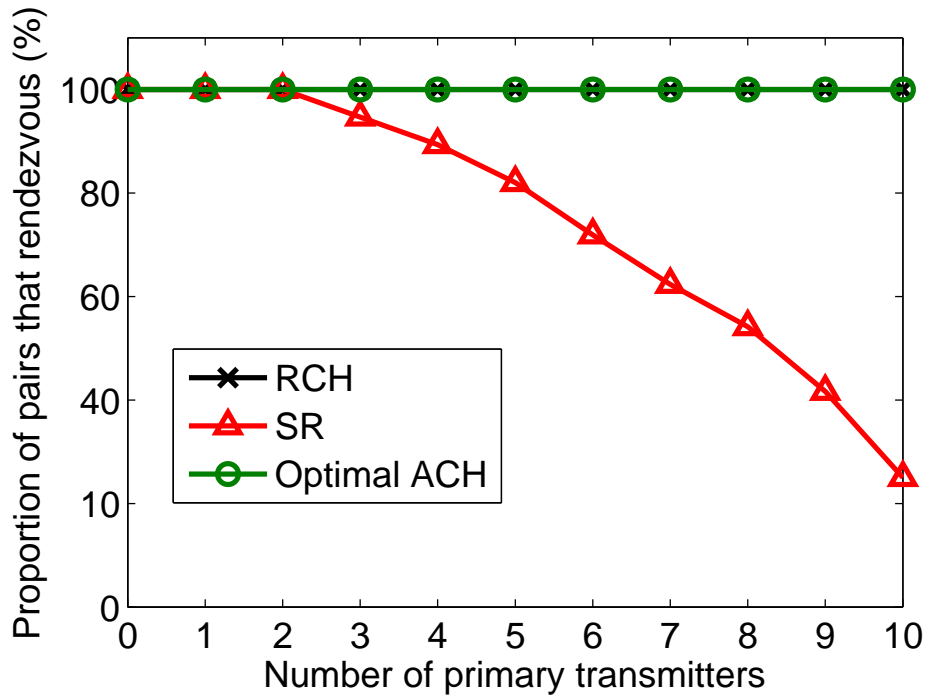

Figure 4.8: The proportion of rendezvous pairs vs. number of primary transmitters.

\subsubsection{Impact of Degree of Overlapping}

To show the impact of degree of overlapping on the rendezvous process, we first simulate a scenario in which primary transmitters continuously transmit on $X$ channels, by setting $b$ equal to the simulation time (or $b=\infty$ ) without any idle period.

Proportion of rendezvous pairs. We use the term rendezvous pair to denote a pair of nodes that are able to rendezvous successfully. We also define the term proportion of rendezvous pairs as the ratio between the number of rendezvous pairs and the total number of node pairs that attempt rendezvous expressed as a percentage. We measured the proportion of rendezvous pairs while varying the number of primary transmitters, and the results are shown in Figure 4.8. As expected, SR showed inferior performance compared to the other two schemes due to the fact that they have small degree-of-overlapping values (see Table 4.1). When the degree of overlapping of a $\mathrm{CH}$ scheme is small, the scheme is more vulnerable against rendezvous failures caused by the presence of PU signals. In 


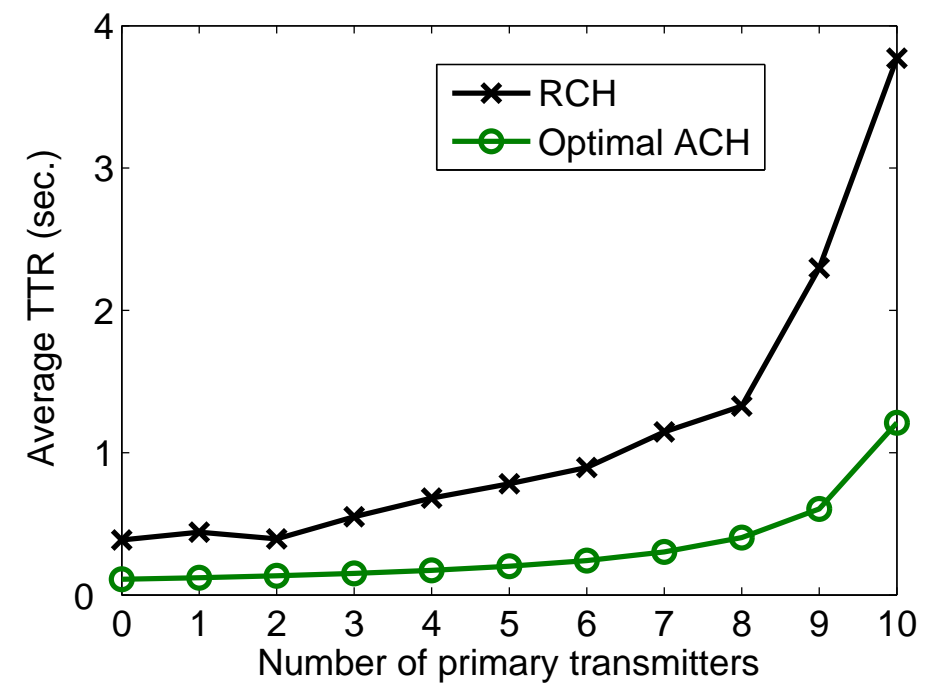

Figure 4.9: Average TTR vs. number of primary transmitters.

contrast, the optimal $\mathrm{ACH}$ scheme has the maximum possible value for the degree of overlapping, viz $N$, which explains its robustness against the aforementioned vulnerability.

ATTR. In Figure 4.9, we compare optimal ACH and RCH in terms of ATTR. Although $\mathrm{RCH}$ has a high proportion of rendezvous pairs (see Figure 4.8), it requires a significantly greater TTR, compared to optimal ACH, to establish rendezvous.

Rendezvous rate. We define the rendezvous rate as the number of successful rendezvous per slot. The rendezvous rate is another measure for quantifying a $\mathrm{CH}$ scheme's ability to establish rendezvous in the presence of PU signals. The measured rendezvous rates for the three $\mathrm{CH}$ schemes are given in Figure 4.10. Note that MRP is a theoretical estimate of the rendezvous rate when all of the $N$ channels are free of primary user signals. The rendezvous rate is an empirically-obtained measure of the number of rendezvous per slot in the presence of PU transmissions. Figure 4.10 shows that the optimal ACH scheme has the highest rendezvous rate among the three $\mathrm{CH}$ schemes. 


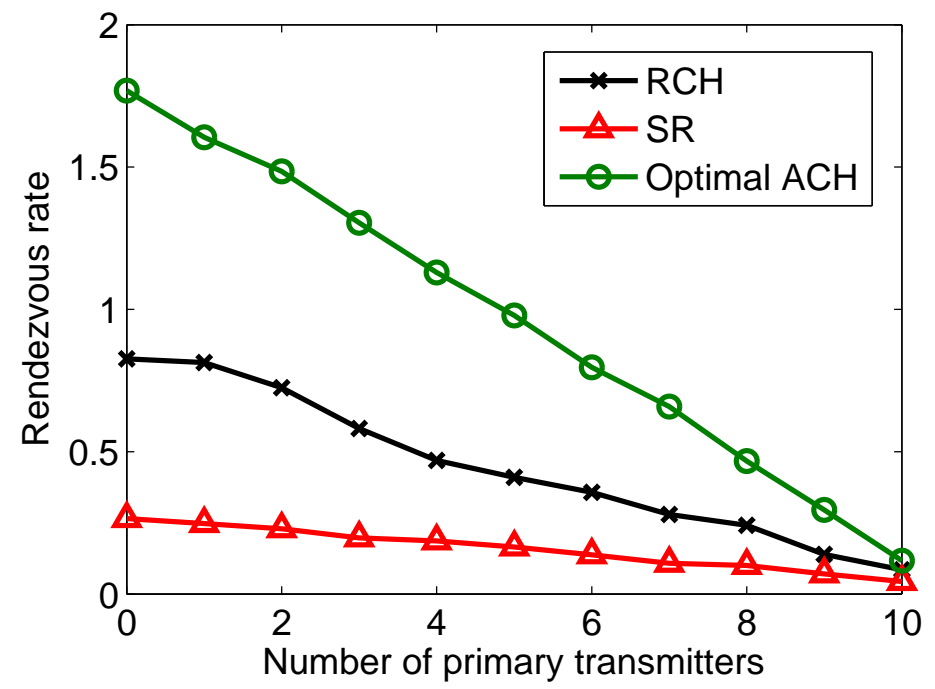

Figure 4.10: Rendezvous rate.

\subsubsection{Impact of Dynamic PU Traffic}

In this set of simulations, we compare the three $\mathrm{CH}$ schemes when the primary user transmission parameters vary dynamically. Specifically, two parameters are varied: the length of the busy period, $b$, and the mean of the idle period, $l$.

In Figures 4.11 and 4.12, we show the average TTR of the three schemes as $l$ is varied while fixing parameters $X$ and $b$. From both of the figures, we can observe that as the spectrum availability of each channel $\left(\frac{l}{l+b}\right)$ increases, the measured ATTR values of all $\mathrm{CH}$ schemes decrease. Note that the performance of SR is inferior to that of $\mathrm{RCH}$ and optimal $\mathrm{ACH}$ due to its long period length $(N(N+1))$ and low degree of overlapping value. From Figure 4.12, we can observe that the increase in $l$, from $l=2$ to $l=8$, while fixing $b=1$ has little effect on decreasing the average TTR of the three $\mathrm{CH}$ schemes. This phenomenon can be attributed to the fact that the availability of the channels is already sufficiently high when $l=2$, which enables most node pairs to readily rendezvous without 


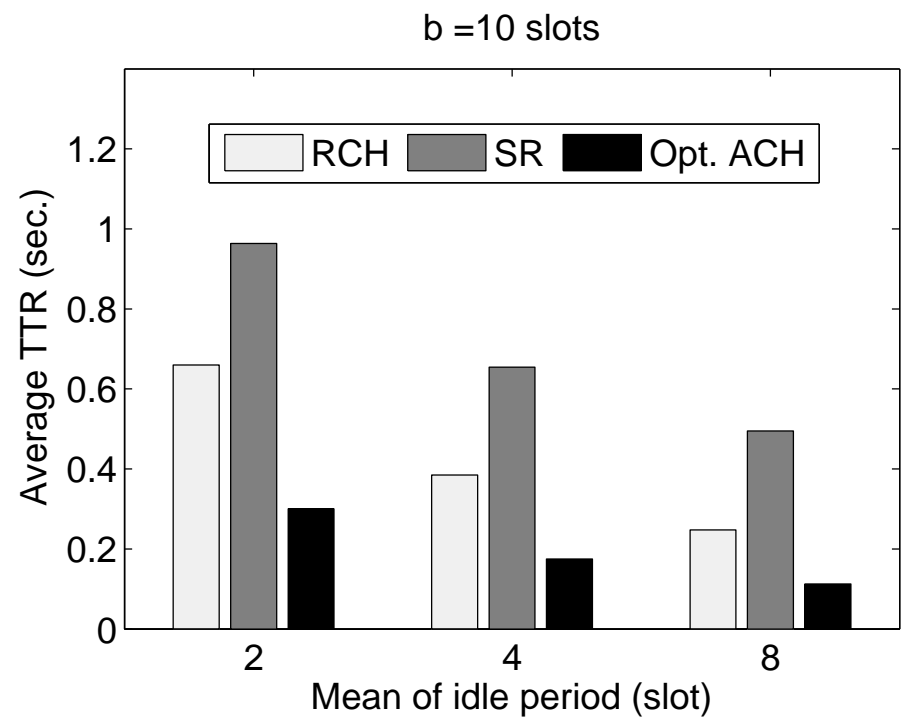

Figure 4.11: Average TTR vs. mean of PU's idle period when $b=10$.

encountering primary user-occupied channels.

Based on our analytical and empirical results, we can make the following conclusions about the relative performance of the asynchronous $\mathrm{CH}$ schemes under consideration: the $\mathrm{CH}$ schemes that have maximum rendezvous diversity (e.g., optimal $\mathrm{ACH}$ ) or provide rendezvous opportunities on every channel (e.g., $\mathrm{RCH}$ ) would outperform the $\mathrm{CH}$ scheme that has a limited degree of overlapping value but a long period length (e.g., SR), in terms of the average TTR.

\subsection{Summary}

In this chapter, we have presented a systematic approach for designing channel hopping $(\mathrm{CH})$ protocols for $\mathrm{CR}$ networks that enable rendezvous between the sender and the receiver even if their clocks are asynchronous. Our approach is novel in that it considers the maximum degree of overlapping, clock synchronization and channel access latency issues 


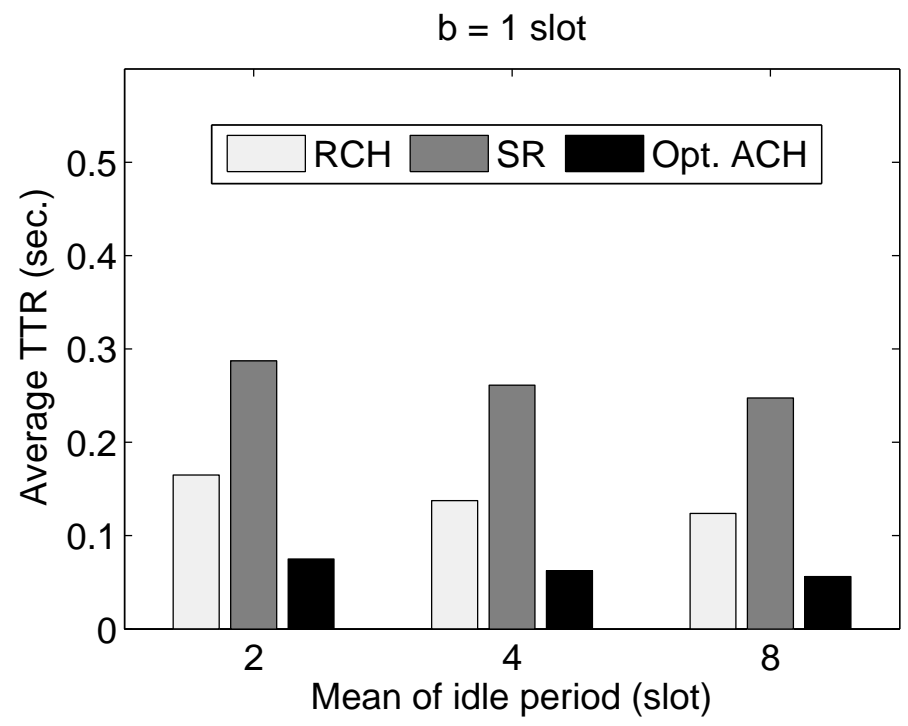

Figure 4.12: Average TTR vs. mean of PU's idle period when $b=1$.

in the design of asynchronous $\mathrm{CH}$ schemes. There are two fundamental challenges in devising an asynchronous $\mathrm{CH}$ scheme for CR networks: (1) establish pairwise rendezvous between two $\mathrm{CH}$ sequences of the sender and the receiver on every available channel; and (2) ensure that two asynchronous $\mathrm{CH}$ sequences achieve rendezvous with an upper bounded time-to-rendezvous (TTR). Using the array-based quorum system, we have devised asynchronous $\mathrm{CH}$ schemes that meet the challenges mentioned above. 


\section{Chapter 5}

\section{Segment-based Channel Assignment in Cognitive Radio Networks}

This chapter is organized as follows. We present a discussion of motivation in Section 5.1. In section 5.2, we provide details on a distributed algorithm for supporting segment-based channel assignment. In section 5.3, we show the simulation results and summarize this chapter in Section 5.4 .

\subsection{Motivation for Segment-based Channel Assignment}

\subsubsection{Segment-based Channel Assignment}

In conventional multi-channel wireless networks, all channels are fixed in terms of frequency and bandwidth [49]. In CR networks, the spectrum opportunities dynamically vary according to the primary users' transmissions. A flow that traverses through areas 
within the range of transmitting primary users experiences dynamically changing channel availability. Thus, it is not possible to assign the same channel to all the links within a flow or component due to the presence of primary signals. This means that flow- and component-based channel assignment algorithms used in conventional wireless networks cannot be applied in CR networks. For this reason, existing channel assignment schemes for CR networks use the link as the granularity of channel assignment. Unfortunately, the link-based approach incurs performance disadvantages due to frequent channel switching. Note that an intersection node that serves multiple links or flows in different channels needs to carry out channel switching. The aforementioned drawbacks include switching delay, synchronization requirement, and scheduling overhead. The latter two drawbacks can be mitigated by employing a control channel. This approach, however, is not desirable for single radio interface network if channel switching overhead is significant, as it requires frequent switching between the control channel and the data channels. Channel assignment approaches for multi-radio networks are discussed in [2].

To address the drawbacks of the link-based approach, we propose a new channel assignment strategy that uses the segment as the granularity of channel assignment. The proposed approach addresses the spectrum variability problem (caused by primary user transmissions) by considering spectrum sensing results in channel assignment decisions, and outperforms the link-based approach by requiring less frequent channel switches.

Before defining the terms segment and segment-based channel assignment, we need to define the term component. A component in a flow graph ${ }^{1}$ is defined as a maximally connected sub-graph [65] such that there exists a path from any node in the sub-graph to all other nodes in the sub-graph. Next, we define the following terms:

\footnotetext{
${ }^{1}$ The set of active links carrying flow traffic in a network.
} 
Definition 8. A segment is defined as the maximal sub-graph of a component in which all nodes in the sub-graph have access to at least one common channel.

Definition 9. The two nodes at both ends of a link that connects two segments of the same component are defined as segment gateway nodes.

The segment-based channel assignment strategy assigns the same channel to all nodes within a segment while conforming to the spectrum utilization rules of the OSS paradigm. The assigned channel is called the operation channel of that segment. An illustration of the segment-based channel assignment strategy is shown in Figure 5.1. Due to the spatial variability in spectrum opportunities, secondary users in different locations may detect different spectrum "white spaces". Suppose there are two primary user transmitters, PU1 and PU2, transmitting in channels 1 and 2, respectively. We assume that the primary network is a TV broadcast network. There is an ad hoc network composed of CR nodes, coexisting with those two primary users. There are two channels in total and two data flows: Flow 1 from node 0 to node 4 and Flow 2 from node 5 to node 6 . Due to PU1 on channel 1 and PU2 on channel 2, the only available channel for nodes $\{0,1,5,6\}$ is channel 2 , while the only available channel for nodes $\{3,4\}$ is channel 1 . Node 2 is outside the interference range of the two primary transmitters, and hence has access to both channels. If Definition 8 is applied to the scenario given in Figure 5.1, then one can see that nodes $\{0,1,5,6\}$ form one segment and nodes $\{2,3,4\}$ form another segment. Note that node 2 can belong to either segment by Definition 8. In Figure 5.1, nodes 1 and 2 are the gateway nodes.

In the above discussions, we have implicitly assumed that the channels of a primary network are equivalent to those of a secondary network. However, this may not be true. 


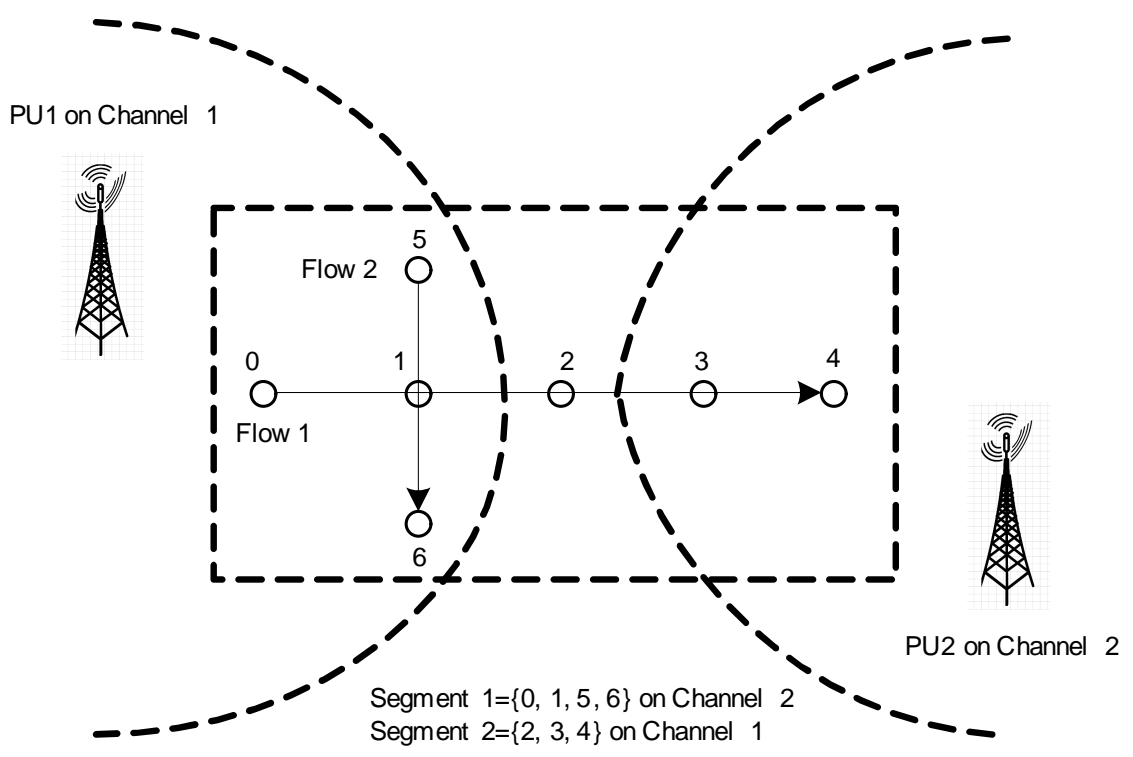

Figure 5.1: An illustration of segment-based channel assignment.

For example, the IEEE 802.22 standard [31] supports channel bonding such that up to 3 TV channels of a primary network can be bonded to one channel for use by the secondary network to support high-bandwidth applications. Since our assumption regarding the channels does not affect our discussions of the proposed segment-based channel assignment scheme, we will continue to make this assumption for the sake of simplicity.

\subsubsection{Performance Considerations}

Link- and flow-based approaches require channel switching when a node serves two links or two flows on different channels. The segment-based approach requires channel switching when two segment gateway nodes on different channels need to communicate with each other. In the following discussions, we compare the segment-based approach with the link-based approach using flow capacity. 


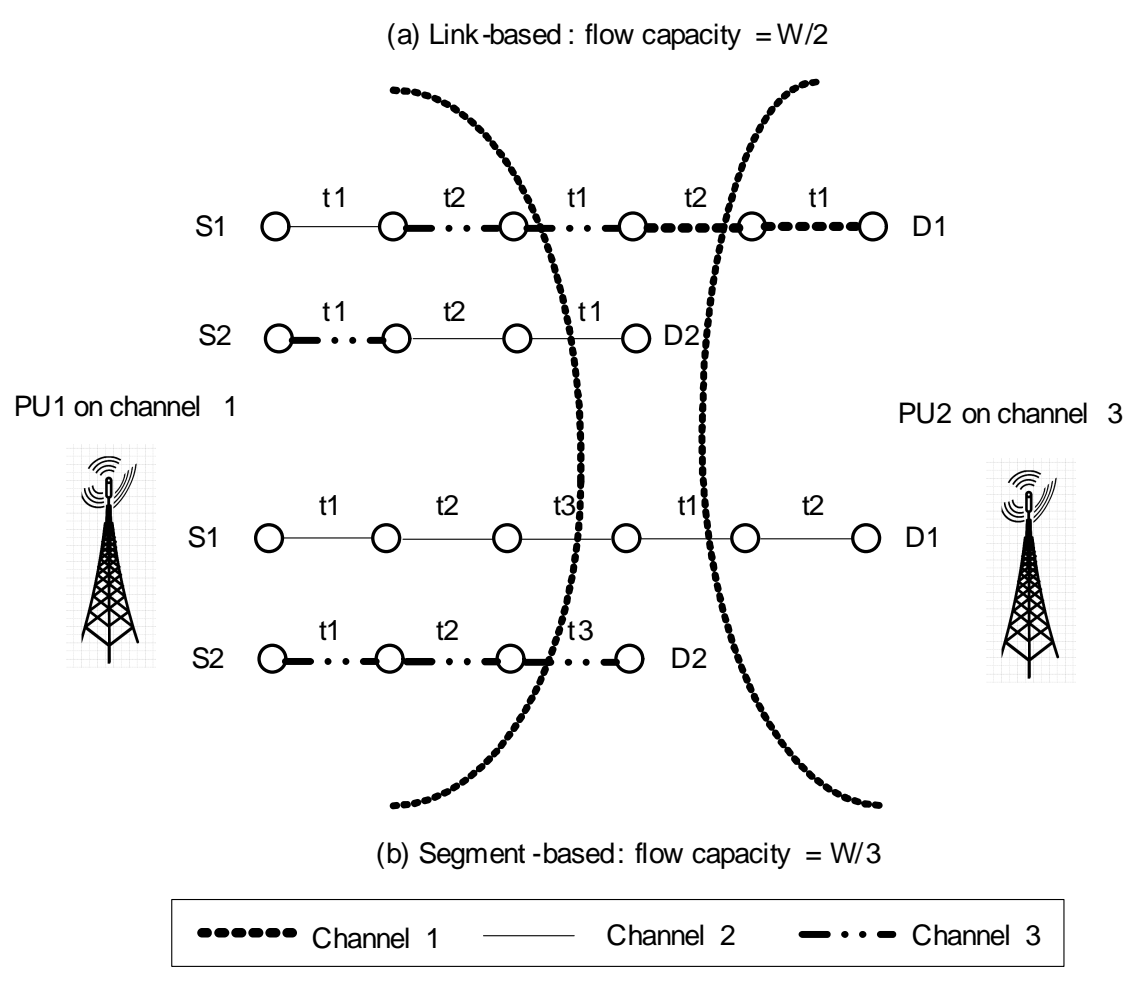

Figure 5.2: Channel and slot assignments for contending flows.

\subsubsection{Flow capacity without channel switching}

In the following analysis, we make the following assumptions: (1) a total of three channels; (2) switching delay is ignored; (3) a two-hop interference region for the secondary network; and (4) links within the same interference region in the same channel are assigned to different slots. We analyze two different cases.

Case 1: Contending flows. We compare the flow capacity of link- and segmentbased approaches for the case of contending flows ${ }^{2}$. Figure 5.2 shows the slot and channel assignments for two contending flows. The operation channels of the two primary users are

\footnotetext{
${ }^{2}$ Two flows are said to be contending, if there is at least one node in the set of active nodes of one flow that is within the interference region of the set of active nodes of the other flow.
} 
also shown. We observe that it is possible to assign the slots in such a way that links within the same contention region are assigned to different slots. In the following discussions, we assume that the link capacity is $W$.

Using the link-based channel assignment, the per-flow capacity is limited to $W / 2$, irrespective of the number of channels and the slot schedule if we assume that each node is equipped with a single, half-duplex radio. In Figure 5.2 (a), the two contending flows each achieve a per-flow capacity of by using channel and slot assignment schemes that utilize three channels and two slots, $t_{1}$ and $t_{2}$.

In the segment-based approach, a single flow may be divided into one or multiple segments. The per-flow capacity is limited by the segment that has the smallest capacity value within the flow. In Figure 5.2 (b), there is only one segment in each flow. The maximum flow capacity of each contending flow is $W / 3$, which is equal to the maximum per-flow capacity of a single flow using segment-based channel assignment.

From the above discussions, we can conclude that the per-flow capacity for both linkand segment-based channel assignment strategies is $O(W)$ for non-intersecting, contending flows.

Case 2: Intersecting flows. Figure 5.3 shows an example of intersecting flows. Node $X$ is the intersection node. Note that when two flows intersect, the per-link capacity is upper bounded by $W / 4$ because the capacity of the links around the intersection node is limited to $W / 4$ due to the fact that each node is equipped with a single half-duplex radio. In the link-based approach, the per-flow capacity is $W / 4$ as shown in Figure 5.3 (a). In the segment-based approach, we need to schedule five slots to avoid interference around the intersection node $X$. Hence, the per-flow capacity is $W / 5$. 


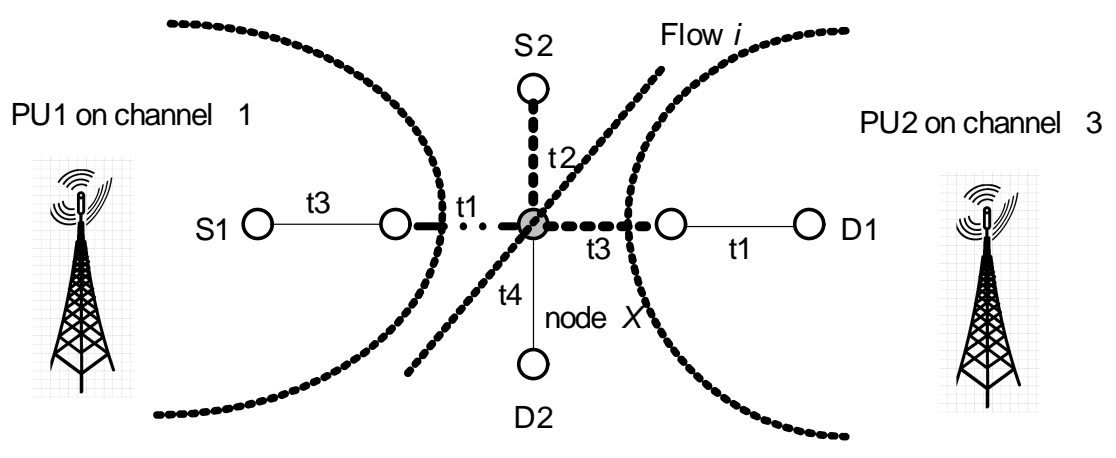

(a) Link-based : flow capacity $=\mathrm{W} / 4$

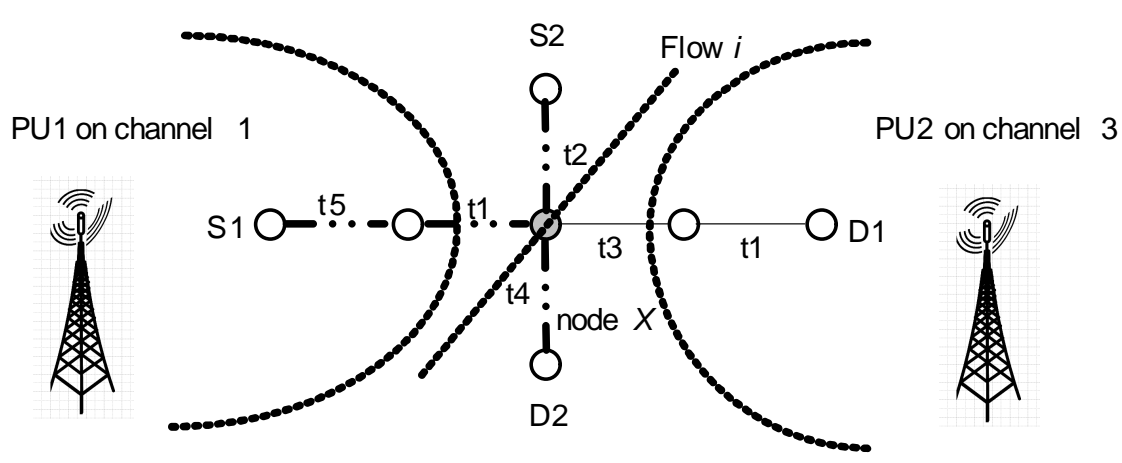

(b) Segment -based: flow capacity $=\mathrm{W} / 5$

$$
\text { ••••• Channel } 1 \_ \text {Channel } 2 \quad \cdots-\text { Channel } 3
$$

Figure 5.3: Channel and slot assignments for intersecting flows.

If we add one more flow (Flow $i$ in Figure 5.3) intersecting at node $X$, two more slots need to be scheduled. Suppose $n$ flows intersect at node $X$, then the aggregate flow capacity of $n$ flows under the link- and segment-based approaches are $W / 2$ and $W /(2+$ $1 / n)$ respectively. From the above equations, we observe that the aggregate flow capacity of $n$ intersecting flows under both channel assignment strategies is $O(W)$.

In the above discussions, we ignored the detrimental effects of channel switching on network performance; we investigate this issue next. 


\subsubsection{Flow capacity with channel switching}

When forwarding a packet, a secondary node needs to switch channels if two neighboring links are assigned to different channels. For a typical 802.11 network interface card, the switching delay is of the order of 80-100 $\mu s$ and the transmission time for a $1 \mathrm{~KB}$ packet at $54 \mathrm{Mbps}$ is $160 \mu s$ [64]. Thus, the switching delay in the aforementioned example is of the same order as the transmission time of a data packet. The switching delay contributes to the increase in the end-to-end delay of each packet's transmission as the switching delay is additive across all nodes that perform switching. According to [39], a wireless network's capacity $C$ degrades as a function of $S /(S+T)$, where $S$ is the switching delay and $T$ is the transmission time. Suppose that there are $x$ intermediate nodes in one flow that serve two neighboring links on different channels. Also, suppose that the flow is divided into $y$ segments. The channel switching delay is $t_{s}$. The additive switching delay for link- and segment-based approaches is given by the following relations: $S_{\text {link }}=x \cdot t_{s}$ and $S_{\text {segment }}=(y-1) \cdot t_{s}$. Typically, the number of segments in a given flow (when using segment-based channel assignment) is much less than the number of nodes that need to perform channel switching (when using link-based channel assignment), thus $y<x$. Assuming other factors are the same, the capacity degradation caused by the switching delay in the link-based approach is greater than that in the segment-based approach. Thus,

we can conclude that $C_{\text {link }}<C_{\text {segment }}$, where $C_{\text {link }}$ represents the estimated capacity under link-based approach and $C_{\text {segment }}$ represents the estimated capacity under segmentbased approach. 


\subsection{Segment-based Channel Assignment}

In this section, we present a distributed approach for realizing the segment-based channel assignment strategy. The proposed approach includes a channel assignment scheme and an adaptive segment maintenance scheme.

\subsubsection{Initial Handshake}

Before communicating with neighboring nodes, a secondary node needs to inform its neighbors about its current operation channel and the list of channels that is available to it. Each node in the network is required to broadcast an initial message (IM) on all of its available channels in a round-robin manner; this procedure is similar to the initial handshake procedure proposed in [69]. An alternative way for the initial handshake is based our proposed rendezvous protocols in Chapters 3 and 4. The IM of node $i$ includes information regarding its available channel set $A_{i}$ and its operation channel. Through the received IMs, a node acquires knowledge about the available channels and the operation channel of each neighboring node. Once the initial handshake is finished, the channel assignment process can start. Note that the proposed channel assignment scheme performs channel assignment, segment formation, and route discovery in an integrated manner. A node needs to broadcast an updated IM to its neighbors every time it changes its operation channel or revises its list of available channels. 


\subsubsection{Channel Assignment}

The proposed channel assignment scheme can be integrated into any routing protocol for wireless ad hoc networks in which the source node of a flow initiates the route discovery process. Here, we take Dynamic Source Routing (DSR) [37] as an example.

\subsubsection{Route request propagation}

The proposed scheme's route request propagation phase is similar to the route request broadcast in DSR, and is described below.

1. A source node broadcasts a route discovery request (RREQ) message on all of the operation channels of its neighboring nodes. The operation channels of the neighbors are obtained from the IMs collected in the initial handshake phase.

2. When an intermediate node $j$ receives the RREQ message, it piggybacks channel contention information on it and broadcasts the modified RREQ message to its neighbors. Specifically, $\left(c l_{j}\left(i_{1}\right), c l_{j}\left(i_{2}\right) \ldots\right)$ is piggybacked on the RREQ, where $c l_{j}(c)$ is the contention level of channel $c \in A_{j}$ perceived by the intermediate node $j$. The contention level of a channel is defined as the number of neighboring nodes that may potentially access this channel. The contention level value is calculated using the information in the IMs exchanged in the initial handshake procedure. As an example, let us calculate the value $c l_{j}(c)$. Suppose an intermediate node $j$ has a set of neighbors represented by $N_{j}$, and each neighbor $r$ has an available channel set $A_{r}$-both values can be obtained from the IMs. For $c \in A_{j}$ and $r \in N_{j}$, we define 
an indicator function as

$$
I_{j}\left(c, A_{r}\right)=\left\{\begin{array}{l}
1, \text { if } c \in A_{r} \\
0, \text { otherwise. }
\end{array}\right.
$$

Then, the contention level of channel $c \in A_{j}$ perceived by node $j$ can be calculated according to

$$
c l_{j}(c)=\sum_{r \in N_{j}} I_{j}\left(c, A_{r}\right) .
$$

The channel contention level values are used in making channel selection decisions.

3. Using the received RREQs, the destination node constructs a set of disjoint paths and selects the shortest path from the set. Here, we assume the shortest-path (i.e., the path with the minimum hop number) selection policy. Then the destination node transmits a route reply (RREP) message back to the source via the reverse path, and prepares for channel assignment and segment formation.

\subsubsection{Route reply and channel assignment}

In a network of secondary nodes, different portions of a route may use different channels due to channel availability influenced by spectrum variability. Suppose $A_{j}$ denotes the list of available channels for node $j$. Then, each route has a corresponding available channel set, represented by $A_{1}, A_{2}, \ldots, A_{k}$, where $k$ is the number of nodes on the route. In order for a route to be valid, relation (5.1) needs to be satisfied:

$$
A_{j} \cap A_{j+1} \neq \emptyset, j=1, \ldots, k-1 .
$$

Relation (5.1) is satisfied when every pair of neighboring nodes on the route has at least one common channel available to both nodes. The RREP message transmitted by the 
destination node traverses through the $k$ nodes towards the source node via the reversepath.

1. Destination node $k$ selects an available channel $i^{*}$ with the smallest contention level value from set $A_{k}$, i.e.,

$$
i^{*}=\arg \min _{i \in A_{k}} \sum_{j=1}^{k} c l_{j}(i) .
$$

Destination node $k$ records its assigned channel as $c h_{k}=i^{*}$. The contention level values are obtained from the piggybacked information in the RREQs. Destination node $k$ creates a new segment $s_{k}$ and assigns itself to this segment. This new segment currently includes only one node, namely node $k$. The destination node piggybacks the 3-tuple $\left(s_{k}, c h_{k}, A_{k}\right)$ in an RREP and sends it on the operation channel of the next node upstream ${ }^{3}$ (i.e., node $(k-1)$ ).

2. When an RREP (with the piggybacked tuple) is received on its operation channel, an intermediate node $j$ checks to see whether it is already associated with an existing segment, $s^{*}$, and assigned to a channel, $c h_{s^{*}}$. i.e., it checks whether $s_{j}=s^{*}$ and $c h_{j}=c h_{s^{*}}$. If node $j$ is already associated with a segment, it ignores the piggybacked information in the RREP. Then, node $j$ notifies all the downstream nodes in its flow that are within the same segment about the channel assignment and the segment association. After receiving the notification message, each notified node resets its assigned channel as $c h_{j}$ and segment association as $s_{j}$. Next, node $j$ piggybacks the tuple $\left(s_{j}, c h_{j}, A_{j}\right)$ in the RREP and sends it to the next node upstream (i.e., node $(j-1))$ on the operation channel of node $(j-1)$.

\footnotetext{
${ }^{3}$ The upstream direction is the direction from the destination to the source. The downstream direction is the opposite of upstream.
} 
3. Suppose that an intermediate node $j$ has not committed to any existing segment and

$$
c h_{j+1}=A_{j}, j=1, \ldots k-1 .
$$

That implies that node $j$ can access the operation channel of the downstream neighbor node $(j+1)$. Thus, node $j$ selects the same channel and associates itself with the same segment as node $(j+1)$, i.e., $c h_{j}=c h_{j+1}$ and $s_{j}=s_{j+1}$. Node $j$ piggybacks the tuple $\left(s_{j}, c h_{j}, A_{j}\right)$ in the RREP and sends it to the next upstream node (i.e., node $(j-1))$ on the operation channel of node $(j-1)$.

4. If node $j$ is not committed to any existing segment and relation (5.3) does not hold, then node $j$ creates a new segment, $s_{j}$, and selects a channel from $A_{j}$ as its operation channel, $c h_{j}$. When selecting a channel, node $j$ applies the same rule that the destination node applied in selecting its operation channel (see step (1) in Section 5.2.2.2). Node $j$ piggybacks the tuple $\left(s_{j}, c h_{j}, A_{j}\right)$ in the RREP and sends it to its upstream node.

5. When the RREP arrives at the source node, the source generates an ACK and sends it to downstream nodes of this route on their current operation channels to notify them that it has received the RREP. When a downstream node receives an ACK from the source, the operation channel and segment association selected by this node take effect. In Figure 5.4, we have applied the aforementioned channel assignment scheme to a simple two-segment network as an example.

Note that our protocol uses the RREP messages to perform channel assignment as opposed to using RREQ messages (cf. [64]). 


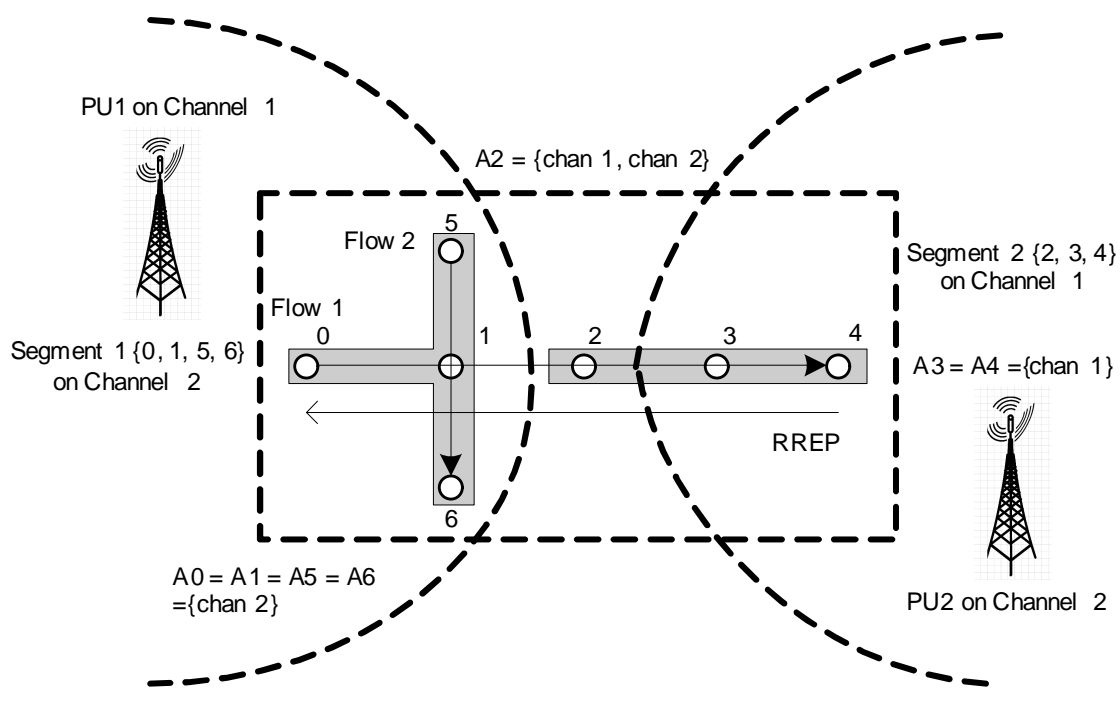

Figure 5.4: Two segments are formed via the segment-based channel assignment approach.

\subsubsection{Segment Maintenance}

The temporal changes in the primary users' utilization of the spectrum would cause changes in spectrum availability for the secondary users, thereby causing some of the secondary nodes to switch to a different channel. This, in turn, would induce changes in the segments within a secondary network. Two scenarios can occur: segment splitting and segment merging. In the following discussions, we discuss both scenarios.

\subsubsection{Segment splitting}

The appearance of a primary user's signal in a spectrum band that is currently occupied by secondary nodes causes those secondary nodes to vacate that band and move to a fallow band by switching to another channel. Note that the appearance of a primary user does not necessarily require all of the nodes within a segment to switch channels; only a subset of the nodes may need to switch. Suppose that an intermediate node $j$ detected the presence 
of a primary user signal and needs to switch to a different channel. Node $j$ triggers a Channel Change message $(\mathrm{CCHG})$ which is propagated towards the source (using the current operation channel). After receiving the $\mathrm{CCHG}$, the source node generates a Route Repair message (RR) and sends it downstream via the current route. RR is forwarded towards the destination until it reaches node $j^{*}$. Node $j^{*}$ is the node closest to the source node among the nodes that generated a CCHG. Node $j *$ initiates a route request propagation procedure to re-establish a new route to the destination. The procedure is the same as the one described in Section 5.2.2. This triggers another round of channel assignments for all nodes downstream of node $j^{*}$. This new channel assignment procedure will lead to the splitting of the current segment into two or more smaller segments.

\subsubsection{Segment merging}

As noted in [64], the performance limitation of link- and flow-based channel assignment schemes is primarily due to the switching delay and overhead incurred when an intersection node serves two links/flows in different channels. Hence, minimizing the number of channel switchings can enhance performance significantly. In the context of the proposed segment-based channel assignment scheme, the number of channel switchings can be reduced when one takes advantage of a scenario in which an active primary user halts transmitting and releases a previously occupied band. By utilizing the recently released band, the secondary network may be able to reduce the number of segments, thus reducing the number of segment gateway nodes that need to carry out channel switching. An exam-

ple is shown in Figure 5.5. In this figure, Primary User 2 (PU2) has halted its transmission, thus releasing Channel 2, and enabling nodes 2, 3, and 4 to use Channel 2. Now, all nodes can be associated with a single segment that uses Channel 2. Comparing Figures 5.4 and 


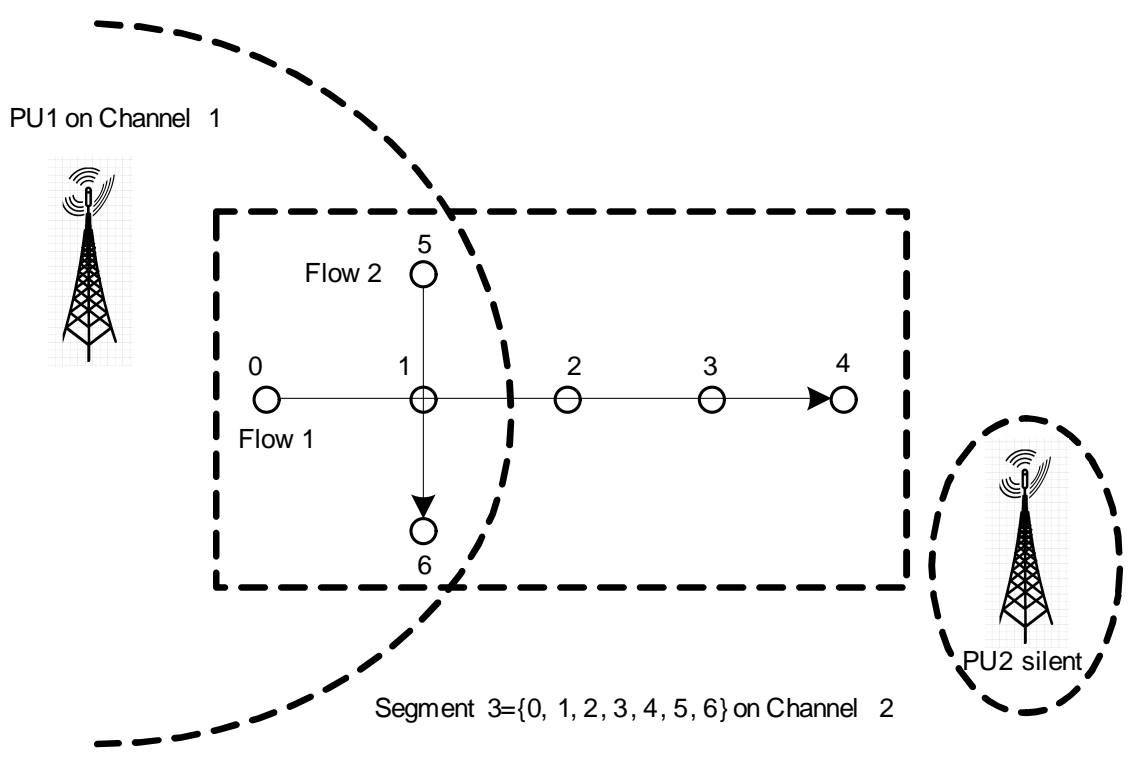

Figure 5.5: Two segments merge into one if primary user 2 is silent.

5.5, we can see that the merging of the two segments into one will no longer require node 1 to carry out channel switching.

As noted above, the primary benefit of segment merging is the reduction of costs associated with channel switching (such as delay and overhead). However, segment merging is not always beneficial. Note that the segment merging process itself requires some nodes to perform a one-time channel switch. In fact, segment merging may even degrade performance when the period of the primary users' on/off cycle is short relative to the lifetime of a flow. For instance, if primary signals occupy and release the current band occupied by the segment's channel with high frequency, then multiple instances of segment merging and splitting may occur during the lifetime of a flow. In such a scenario, the overhead of segment merging/splitting would outweigh the benefits of segment merging, thus leading to poor performance. An interesting avenue for future research is investigating the tradeoffs of segment merging and its relationship to the performance of the secondary network. 


\subsection{Performance Evaluation}

\subsubsection{Simulation Setup}

Throughout this section, we compare the proposed segment-based channel assignment scheme with the link-based channel assignment scheme. The simulations are carried out for a $1000 m \times 1000 m$ square area with 50 secondary nodes placed randomly via a uniform distribution. All nodes are stationary. The transmission range of each secondary node is $250 \mathrm{~m}$. We assume the existence of two primary signal transmitters-one transmitting in channel 1 and located at coordinates $(0,500)$, and the other one transmitting in channel 2 and located at coordinates $(1000,500)$. The default channel capacity is $1 \mathrm{Mbps}$, and the channel switching delay has a constant value of $100 \mu \mathrm{s}$. To simulate the proposed channel assignment scheme, we modified DSR to support channel assignments at the granularity of segments. In the simulated link-based approach, a link is assigned to a channel that has the smallest contention level value. For comparing the two channel assignment approaches, two evaluation metrics were used: average throughput and accumulative end-to-end delay. All results shown here are the results of averaging 10 simulation runs for each experiment.

\subsubsection{Simulation Results}

The simulation result shows superiority of our segment-based approach in performance over the link-base approach. 


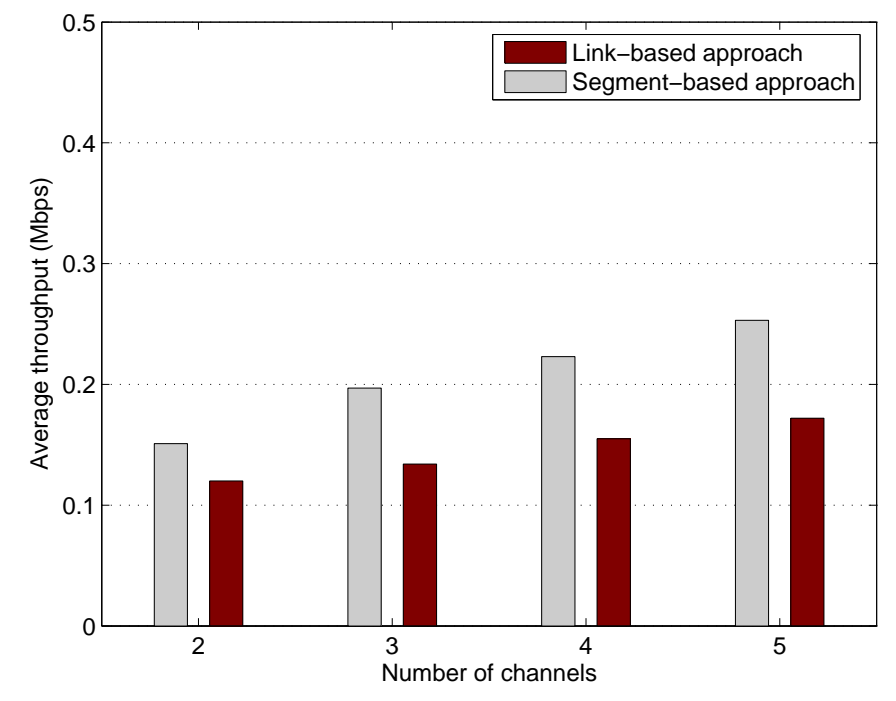

Figure 5.6: Average throughput vs. number of channels.

\subsubsection{Invariant primary user transmission}

We assume that the two primary users transmit during the entire duration of the simulation time interval. The total number of available channels is five. We compare the two channel assignment approaches using the average throughput of 10 UDP flows while varying the number of available channels. The results are shown in Figure 5.6. We varied the total number of channels from 2 to 5.

\subsubsection{Segment merging in the presence of variant primary user transmission}

To maintain an adequate level of performance, it is expected that the secondary network will choose to use licensed spectrum bands in which primary users' transmission pattern is not extremely dynamic (i.e., do not change very frequently). Thus, here we do not consider the case in which the primary users' transmission pattern varies frequently. We 


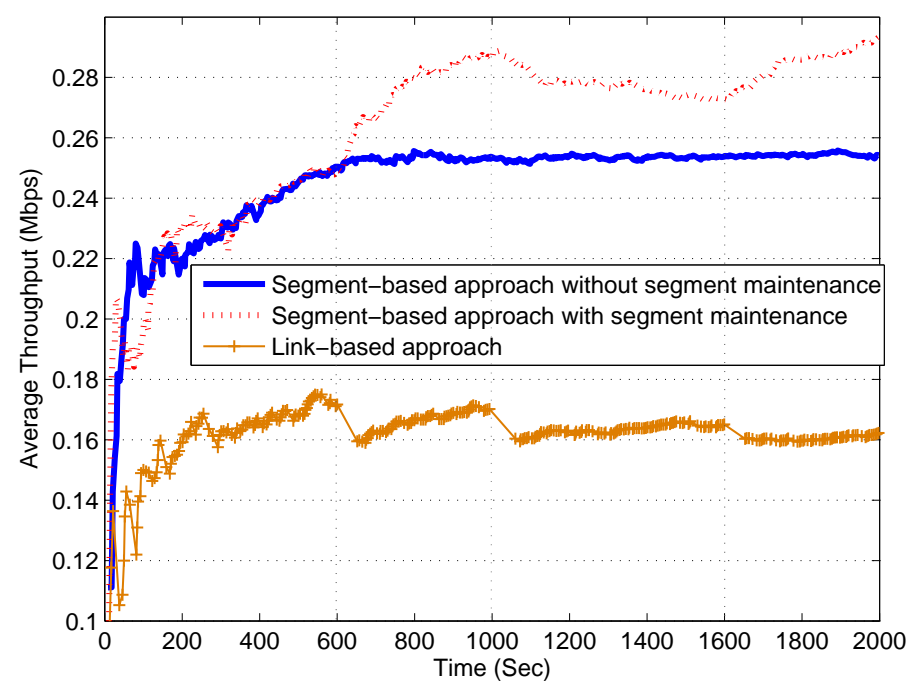

Figure 5.7: Comparisons on the average throughputs of three protocols.

carried out a set of simulation experiments in which the primary user PU2 (operating in channel 2) has the following transmission schedule: it starts transmitting at the time point $0 \mathrm{sec}$; turns off at the 600 -th sec; restarts transmitting at the 1000 -th sec; and turns off again at the 1600-th sec. The total simulation time is 2000 seconds. We compared the average throughput of three channel assignment schemes: segment-based approach without segment maintenance, segment-based approach with segment maintenance (i.e., segment merging and segment splitting), and link-based approach. The second approach performs segment merging when PU2 turns off to utilize the freed channel, and performs segment splitting when PU2 turns back on to avoid interfering with the primary signal transmission.

In Figure 5.7, we observe that the segment merging process used by the second channel assignment scheme causes the average throughput to improve. We can clearly see the throughput curve of the second scheme follow a positive slope during PU2's two off time 
periods (i.e., $[600,1000]$ and $[1600,2000])$. During the interval $[1000,1600]$, the throughput curve of the segment-based channel assignment scheme with segment maintenance follows a negative slope due to the delay and overhead involved in segment splitting.

\subsection{Summary}

In this chapter, we have considered the channel assignment problem in single radio interface, $\mathrm{CR}$ ad hoc networks. We have proposed a novel channel assignment scheme that assigns channels at the granularity of segments. In the OSS paradigm, the temporal and spatial spectrum variability caused by the primary users' spectrum utilization adds another dimension of complexity to the problem of channel assignment. Existing channel assignment approaches—-such as link-based and component-based approaches—do not consider spectrum variability because they were originally designed for conventional wireless networks. Therefore, those approaches do not offer the best trade-off in terms of incumbent coexistence and network performance in the OSS model. The proposed channel assignment scheme is significantly simpler than the existing approaches, and offers practical benefits. Using simulation results, we have demonstrated that the segment-based channel assignment scheme outperforms the link-based channel assignment approach under realistic network conditions. 


\section{Chapter 6}

\section{A Coexistence-aware Spectrum Sharing Protocol}

This chapter is organized as follows. In Section 6.1, we propose the Coexistence-aware Spectrum Sharing (CASS) protocol, and we evaluate the performance of CASS in Section 6.2. We discuss related work in Section 6.3 and conclude this chapter in Section 6.4.

\subsection{The CASS Protocol}

\subsubsection{Basic Assumptions}

Incumbent traffic. We assume that the presence or absence of incumbent users' signals on a channel, say channel $i$, can be modeled as a continuous-time "ON/OFF" Poisson process [28], where inter-arrival times of consecutive incumbent signals are exponentiallydistributed with a rate parameter. Let the random variable $V_{i}$ denote the length of an 
incumbent's idle interval (incumbent idle time period) on channel $i$. Similarly, let the random variable $U_{i}$ denote the length of an incumbent's busy interval (incumbent busy time period) on channel $i$. Suppose that $E\left[V_{i}\right]=v_{i}$ and $E\left[U_{i}\right]=u_{i}$. As shown in [38], the value of $v_{i}$ and $u_{i}$ can be estimated using spectrum sensing results. The probability that channel $i$ is free of incumbent users' signals is

$$
\alpha_{i}=\frac{v_{i}}{v_{i}+u_{i}}
$$

QoS requirement. The spectrum contention process would require the coexisting networks to exchange potentially sensitive information-such as QoS requirements, traffic load, and network characteristics - to negotiate and resolve the contention among them. We need a measure to quantify the traffic load of a coexisting network. For this purpose, we use $r(x)$ to denote the minimum number of channels required by a BS $x$ to satisfy the QoS of its workload.

Inter-BS communication. 802.22 defines inter-BS communication methods: overthe-air and over-the-backhaul in support of the inter-BS communication. These features enable a BS to exchange control information with neighboring BSs in a timely and efficient manner.

\subsubsection{Dynamic Switching between the Two Spectrum Sharing Modes}

CASS supports two spectrum sharing modes: non-exclusive spectrum sharing and exclusive spectrum sharing. It can switch from one mode to the other depending on the channel conditions. CASS performs channel evaluation to determine when to switch from one mode to the other. In this channel evaluation process, CASS needs to calculate the channel capacity in each of the two spectrum sharing modes. 


\subsubsection{Channel capacity in non-exclusive spectrum sharing mode}

If the renter $\mathrm{BS}$ shares channel $i$ with its neighbors, self-interference may cause packet collisions. Let $\gamma(i)$ denote the maximum achievable SIR perceived by the renter on the selected channel $i$. Let $e(i)$ denote the packet error rate on the selected channel at a receiver (a CPE) in the renter's cell. The capacity of channel $i$ in non-exclusive spectrum sharing can be expressed as

$$
C_{N}(i)=\alpha_{i} \cdot(1-e(i))
$$

In [53], Shellhammer describes a way of estimating $e(i)$ based on $\gamma(i)$ : given the modulation method, the symbol error rate (SER) can be estimated based on $\gamma(i)$; then, $e(i)$ can be calculated based on the SER.

\subsubsection{Channel capacity in exclusive spectrum sharing mode}

If exclusive spectrum sharing of the selected channel is necessary, a contention phase is periodically scheduled so that the channel contention procedure can be invoked. Let $T$ denote the duration of a channel contention period. At the end of every contention period, a contention phase of length $S$ is scheduled, as shown in Figure 6.1. $S$ must be long enough to perform channel contention-related operations, such as determining the target channel(s), performing channel contentions (via the exchange of inter-BS beacons),

and preparing to resume transmissions on the new channels (if new channels have been acquired or if channel switches have occurred).

The capacity of channel $i$ in exclusive spectrum sharing can be calculated as

$$
C_{E}(i)=\alpha_{i} \cdot \frac{T_{X}(i)}{v_{i}}
$$




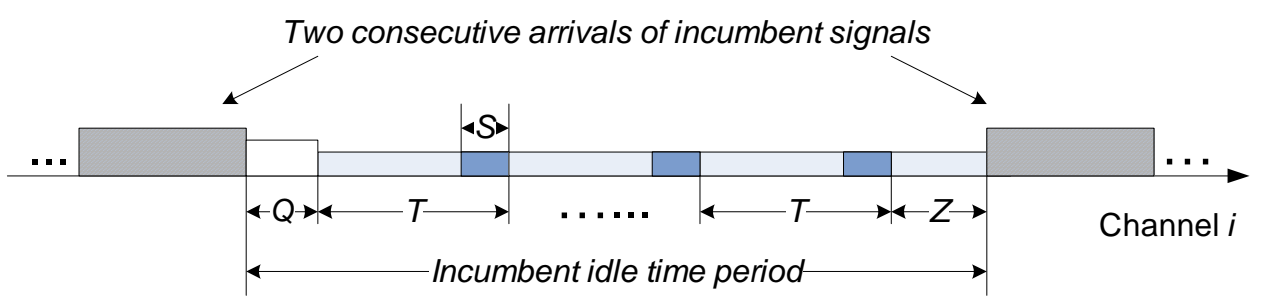

Figure 6.1: Incumbent idle time period and channel contention periods.

where $T_{X}(i)$ is the expected maximum transmission time for 802.22 entities on channel $i$ during an incumbent idle time period.

802.22 entities cannot immediately start to access a channel once spectrum sensing determines that the channel is fallow. A quiet period of length $Q$ is needed for performing spectrum sensing and channel setup before an 802.22 entity can access the channel, as shown in Figure 6.1. Let the random variable $\tau$ and random variable $L_{i}$ denote the length of the incumbent idle time period and the length of an 802.22 entity's maximum transmission time on channel $i$ (during an incumbent idle time period), respectively. One of two possible scenarios can occur:

- if $\tau \leq Q, L_{i}=0$

- If $\tau>Q$, the incumbent idle time period is composed of a quiet period, $\left\lfloor\frac{\tau-Q}{T}\right\rfloor$ contention periods and a residual time interval $Z$ as shown in Figure 6.1, where $0 \leq Z<T$.

Let $p_{F N}$ denote the false negative ${ }^{1}$ probability of spectrum sensing in the contention source's cell. Now we can calculate the expected maximum transmission time on channel

\footnotetext{
${ }^{1}$ This type of error occurs when the spectrum sensing results erroneously indicate that a channel is free of incumbent signals when in fact the opposite is true.
} 


$$
\begin{aligned}
& T_{X}(i)=E\left[L_{i}\right]=0+\left(1-p_{F N}\right) \cdot \\
& \qquad \int_{Q}^{\infty}\left(\left\lfloor\frac{\tau-Q}{T}\right\rfloor \cdot(T-S)+\min (Z, T-S)\right) \cdot p_{\tau}(i) d \tau,
\end{aligned}
$$

where $p_{\tau}(i)$ is the probability that there is no incumbent signal arrival in channel $i$ during the time duration $\tau$. This value can be calculated using the following equation:

$$
p_{\tau}(i)=\int_{\tau}^{\infty} \frac{1}{v_{i}} \cdot e^{-\frac{u}{v_{i}}} d u=e^{-\frac{\tau}{v_{i}}}
$$

\subsubsection{An approach of switching between two modes}

In 802.22, the non-exclusive spectrum sharing of the selected channel is attempted first. As stated in Rule 2, if the maximum achievable SIR on the selected channel is not sufficiently high, 802.22 prescribes exclusive spectrum sharing. However, 802.22 fails to give specific guidelines on when to use exclusive sharing. We propose one possible approach for determining when to use exclusive spectrum sharing. CASS determines which spectrum sharing mode to operate in, for a given channel $i$, by comparing $\gamma(i)$ with the required SIR threshold parameter $\gamma^{*}(i)$. To obtain the value of $\gamma^{*}(i)$, we need to first solve for $e(i)$ in the equation $C_{N}(i)=C_{E}(i)$; and then find the SIR value corresponding to the solved $e(i)$, which is used as the value of $\gamma^{*}(i)$. CASS determines the spectrum sharing mode as follows:

1. If $\gamma(i) \geq \gamma^{*}(i)$, CASS operates in the non-exclusive spectrum sharing mode. The benefits of non-exclusive sharing (e.g., low control overhead) outweigh the benefits of exclusive sharing (e.g., no self-interference). 
2. If $\gamma(i)<\gamma^{*}(i)$, CASS operates in the exclusive spectrum sharing mode. The benefits of exclusive sharing outweigh the benefits of non-exclusive sharing.

\subsubsection{The Channel Selection Mechanism}

The channel selection criterion in 802.22's mechanisms (Rule 1) implies that the renter BS does not consider any incumbent coexistence issues. It may not be suitable in environments where the appearance of incumbent signals are frequent and unpredictable—such a scenario would likely occur in areas where Part 74 devices operate. After the incumbent signals on active channels have been detected in a cell, the BS and CPEs need to vacate those channels as soon as possible and find suitable replacement channels. The incumbent users in the cell's vicinity are also likely to experience some level of interference. Instances of such undesirable situations can be reduced by considering the incumbent coexistence requirement during the channel selection process. We propose to use an incumbent protection criteria-probability of interfering with incumbent signals on an 802.22 channel. CASS prescribes that the renter BS should select an 802.22 channel that has the lowest probability of interfering with incumbent signals.

For a BS $x$ in need of spectrum, let $N(x)$ denote the set of neighboring BSs of $x$. Let $C(y)$ denote BS $y$ 's set of candidate channels. BS $x$ 's grand candidate channel set is

$$
G(x)=\bigcup_{y \in N(x)} C(y) .
$$

For channel $i$ and $\mathrm{BS} y$, let $r_{i}^{y}$ denote the probability that BS $y$ schedules data transmissions on channel $i$ in a given time interval. The value of $r_{i}^{y}$ for any channel $i$ is determined by the BS $y$ itself. When $r_{i}^{y}=0$, this means that channel $i$ is a candidate channel for BS $y$ or 
channel $i$ is not allocated to BS $y$. Neighboring BSs exchange information on each other's $r_{i}^{y}$ value using inter-cell beacons ${ }^{2}$.

We define the channel accessibility of channel $i$ for the renter BS $x$, represented by notation $\varphi_{x}(i)$, as the probability that BS $x$ can access channel $i$ with no incumbent or selfinterference in a given time interval. We can compute $\varphi_{x}(i)$ using the following equation:

$$
\varphi_{x}(i)=\alpha_{i} \prod_{y \in N(x)}\left(1-r_{i}^{y}\right) .
$$

We define the set of accessible candidate channels for the BS $x$ as

$$
R(x)=\left\{i \mid \varphi_{x}(i)>\eta, i \in G(x)\right\}
$$

where $\eta$ is an adjustable parameter.

To comply with CASS's channel selection rule, BS $x$ selects channel $s$ such that

$$
s=\arg \min _{i \in R(x)}\left\{p_{\text {int }}(i)\right\}
$$

where $p_{\text {int }}(i)$ is the probability of interfering with incumbent signals on channel $i$. Let $t$ denote the expected length of secondary user packets. If $v_{i}$ is known, $p_{\text {int }}(i)$ can be calculated as

$$
p_{\text {int }}(i)=1-\int_{t}^{\infty} \frac{1}{v_{i}} \cdot e^{-\frac{u}{v_{i}}} d u=1-e^{-\frac{t}{v_{i}}}
$$

CASS's channel selection technique minimizes the probability of interfering with incumbent signals (as prescribed by (6.3)) subject to the constraint on channel accessibility (as prescribed by (6.1)).

\footnotetext{
${ }^{2}$ In 802.22, a quiet period in every 802.22 frame is used for 802.22 entities to exchange inter-cell beacons. Information about the cell's candidate channel sets and active channel sets is contained in the beacons.
} 


\subsubsection{The Channel Contention Procedure}

In ODSC's CCN-based channel contention procedure, every BS has the equal probability of winning the target channel, which is $1 / k$ ( $k$ is the number of BSs participating in the contention process). This implies that a contention source that has a high QoS requirement is unlikely to win the needed channels when there are a large number of competing BSs. To address this problem, instead of using CCN, CASS employs the Contention Priority Number $(C P N)$ to resolve pair-wise contentions.

\subsubsection{Contention priority number}

Let $H(i)$ define set of BSs that contend for channel $i$, and let $p_{x}$ denote the CPN of BS $x$. In CASS, every BS $x \in H(i)$ that contends for channel $i$ will broadcast its QoS requirement $r(x)$ to neighboring BSs.

After collecting the QoS requirements of competing BSs, every BS is able to determine the contention window size $W$ as follows.

$$
W=\sum_{x \in H(i)} r(x) .
$$

Every $\mathrm{BS} x \in H(i)$ selects its $\mathrm{CPN} p_{x}$ from the range $[0, W-1]$ at random.

\subsubsection{Token assignment}

After the contention windows size $W$ is determined, let $\mathcal{K}=\{0,1,2 \ldots, W-1\}$ be a set that contains $W$ tokens. Every BS $x \in H(i)$ picks a number of $r(x)$ tokens from $\mathcal{K}$ at random. Let $K_{x}$ denote the set of tokens picked by BS $x$. As a result, all tokens in $\mathcal{K}$ are 
assigned to $k$ competing $\mathrm{BSs}$, i.e.,

$$
\mathcal{K}=\bigcup_{x \in H(i)} K_{x}
$$

\subsubsection{Contention resolution rule}

CASS's contention resolution rule is straightforward: the winner BS is the one who has picked the winner token during the token assignment phase. Let $\omega$ denote the winner token number, and we introduce a simple algorithm that selects the winner token.

- Every BS $x \in H(i)$ broadcasts its selected CPN to all competing BSs.

- After collecting $(k-1) \mathrm{CPN}$ values from other BSs, each BS is able to calculate the winner token as follows.

$$
\omega=\sum_{x \in H(i)} p_{x} \quad(\bmod W)
$$

- The winner BS $x^{*}$ is the one that has picked the token $\omega$ during the token assignment phase, i.e., $\omega \in K_{x^{*}}$.

The winner BS $x^{*}$ adds channel $i$ to its active channel set. The contention destination BS removes the contended channel $i$ from its available channel list. Meanwhile, non-winner BSs that are involved in the channel contention process send beacons to its neighboring BSs to notify them about the result of the channel contention.

Next, we show that the above contention resolution rule guarantees the weighted fairness, i.e., the winning probability of BS $x$ is proportional to its QoS requirement $r(x)$. 


\subsubsection{Weighted fairness}

Suppose BS $x \in H(i)$ has a QoS requirement $r(x)$. The CPN of BS $x, p_{x}$, is a random variable that uniformly distributed over the set $[0, W-1]$. Thus,

$$
\operatorname{Pr}\left\{p_{x}=v\right\}=\frac{1}{W}, \forall v \in[0, W-1]
$$

Let $S$ denote the summation of all CPNs of BSs in $H(i)$ except $p_{x}$, that is

$$
S=\sum_{y \neq x, y \in H(i)} p_{y} .
$$

Let $L=\left(p_{x}+S\right)$. Since

$$
\begin{aligned}
L & =\left(p_{x}+S\right) \quad(\bmod W) \\
& =p_{x} \quad(\bmod W)+S \quad(\bmod W),
\end{aligned}
$$

then we have $\forall u \in[0, W-1]$,

$$
\begin{aligned}
\operatorname{Pr}\{L=u\} & =\sum_{v=0}^{W-1}\left(\operatorname{Pr}\left\{p_{x}=v\right\} \cdot \operatorname{Pr}\{S=(u-v) \quad(\bmod k)\}\right) \\
& =\sum_{v=0}^{W-1}\left(\frac{1}{W} \cdot \operatorname{Pr}\{S=(u-v) \quad(\bmod k)\}\right) \\
& =\frac{1}{W} \cdot \sum_{v=0}^{W-1}(\operatorname{Pr}\{S=(u-v) \quad(\bmod k)\}) \\
& =\frac{1}{W} .
\end{aligned}
$$

Since BS $x$ has $r(x)$ tokens, the probability that one of BS $x$ 's picked tokens is the winner token is $\frac{r(x)}{W}$, which is also the probability that BS $x$ wins the contention. As a result, BS $x$ will have a higher winning probability than BS $y$ if $r(x)>r(y)$. 


\subsection{Performance Evaluation}

In this section, we compare CASS with 802.22 's inter-BS resource sharing mechanisms via simulation results. All of the scenarios we considered involve multiple overlapping cells. In each cell, there is one BS and ten CPEs. Every BS requires five channels to satisfy the QoS of its admitted workload. BSs of overlapping cells are synchronized by the periodic transmission of inter-cell beacons. The simulation parameter values were chosen to be consistent with those used in the simulation experiments in [33], and they are given in Table 6.1. The 802.22 Working Group has suggested that a variation of the Hata model is the most appropriate propagation model for studying 802.22 [32]. The Hata model [50] represents the urban area propagation loss as a standard formula and supplies correction equations to model suburban and open rural areas. In our simulations, we use a variation of the Hata model for open rural areas. We simulated three inter-BS spectrum sharing protocols: 802.22 Resource Renting, 802.22 ODSC, and CASS. Note that Resource Renting is a pure non-exclusive spectrum sharing protocol; ODSC is a pure exclusive spectrum sharing protocol; and CASS can operate in either spectrum sharing mode, switching from one mode to the other when the channel conditions warrant it. We created our own event-driven simulator in $\mathrm{C}$, and each simulation result is the average value of ten simulation runs.

We use the notation $\lambda_{i}$ to denote the number of incumbent signal arrivals on channel $i$ during one superframe ${ }^{3}$, assuming that the incumbent signals follow a Poisson arrival process-hereafter, we'll simply refer to this value as the incumbent transmission rate. We assume that there is at most one incumbent transmitter per channel per cell.

\footnotetext{
${ }^{3} \mathrm{An} 802.22$ superframe is composed of 16 frames.
} 
Table 6.1: Default simulation parameter values.

\begin{tabular}{|c||c|}
\hline \multicolumn{1}{|c||}{ Simulation Parameters } & Values \\
\hline \hline Total licensed spectrum band & $54-806 \mathrm{MHz}$ \\
\hline Bandwidth of a licensed channel & $8 \mathrm{Mbps}$ \\
\hline Number of licensed channels & 10 \\
\hline BS transmission radius & $30 \mathrm{Km}$ \\
\hline TV transmission receiving radius & $30 \mathrm{Km}$ \\
\hline Modulation method & QPSK \\
\hline Effective Antenna height of the BS & $30 \mathrm{~m}$ \\
\hline Effective antenna height of the CPE & $9 \mathrm{~m}$ \\
\hline Channel switch delay & $10 \mathrm{~ms}$ \\
\hline 802.22 frame size & $40 \mathrm{~ms}$ \\
\hline
\end{tabular}

\subsubsection{The 3-BS Scenario}

We simulated a scenario with three overlapping 802.22 cells. The topography of the cells is shown in Figure 2.3. The distance between two neighboring BSs is $d \mathrm{Km}$. In this simulation experiment, the initial channel assignments are given as: BS $a$ 's active channel set is $\{1,2,3,4,9\}$ and its candidate channel set is $\{10\}$. BS c's active channel set is $\{1,2,3,4,10\}$ and its candidate channel set is $\{9\}$. BS $b$ has only an active channel set $\{5,6,7,8\}$, and it is in spectrum shortage. The grand candidate channel set of BS $b$ is $G(b)=\{9,10\}$. Let $\lambda_{i}=0, i=1,2, \ldots, 9$, and $\lambda_{10}=1 / 2$.

Figure 6.2 shows a plot of the throughput of BS $b$ (renter) vs. the distance between neighboring BSs, $d$. As expected, when $d$ is small $(<50 \mathrm{Km})$, CASS and ODSC outperformed Resource Renting. When the distance between neighboring BSs is small, the 


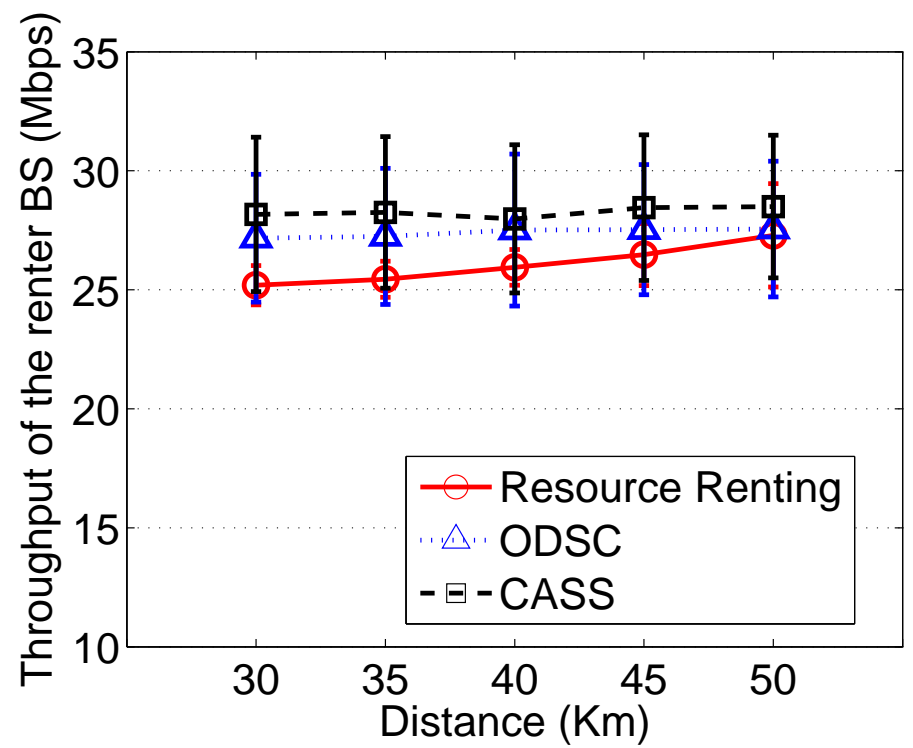

Figure 6.2: Throughput of BS $b$ vs. distance $d$ in the 3-BS scenario.

SIR measured at the 802.22 entities (BS and CPEs) of the renter cell is low due to selfinterference. In such a situation, a non-exclusive spectrum sharing scheme, such as Resource Renting, would be expected to perform poorly. When $d=50 \mathrm{Km}$, Resource Renting's performance was comparable to that of ODSC because the effects of self-interference were insignificant at this distance.

Figure 6.2 shows that CASS outperforms both Resource Renting and ODSC irrelevant of the value of $d$. We can explain this result by recalling the fact that CASS attempts to select a channel that is most underutilized by the incumbent users by requiring the renter BS to consider spectrum sensing results during the channel selection process; in contrast, the other two spectrum sharing schemes do not consider spectrum sensing results. When either Resource Renting or ODSC is used, the likelihood that an incumbent signal appears in the active channel(s) is higher compared to CASS. Obviously, the appearance of incumbent signals in an active channel degrades the renter cell's throughput as this requires costly 


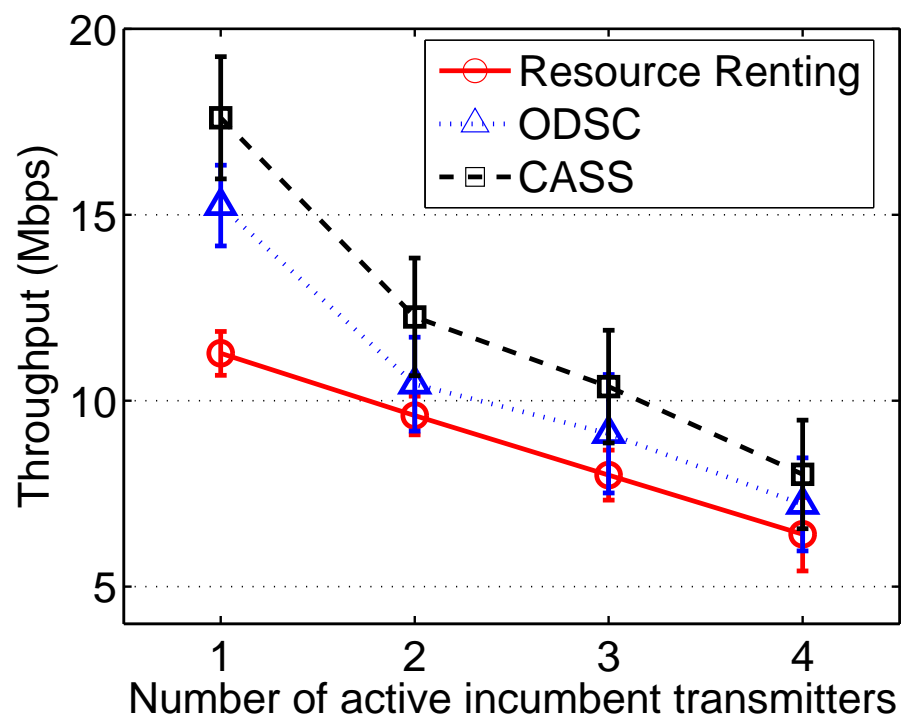

Figure 6.3: Throughput vs. Number of active incumbent transmitters in the 9-BS scenario. channel switches. When CASS is used, BS $b$ selects channel 9 from its grand candidate channel set because $\lambda_{9}<\lambda_{10}$. In practice, a renter BS can use the following procedure to utilize spectrum sensing results when selecting a channel: (i) Using a compiled record of recent spectrum sensing data and a maximum likelihood estimator, estimate the expected incumbent idle time for each channel (see [38] for details); (ii) Using (6.3) and the values obtained in the first step, calculate the probability of interfering with incumbent signals for each channel; and (iii) Select a channel using (6.2).

\subsubsection{The 9-BS Scenario}

We simulated a network topography with 9 overlapping cells in a $100 \times 100 \mathrm{Km}$ area. All channels are randomly assigned to the BSs. Figure 6.3 shows plots of average throughput per cell vs. the number of incumbent transmitters per cell. The incumbent transmission rate was fixed to $1 / 4$ for all incumbent transmitters. The performance gap is seen between 


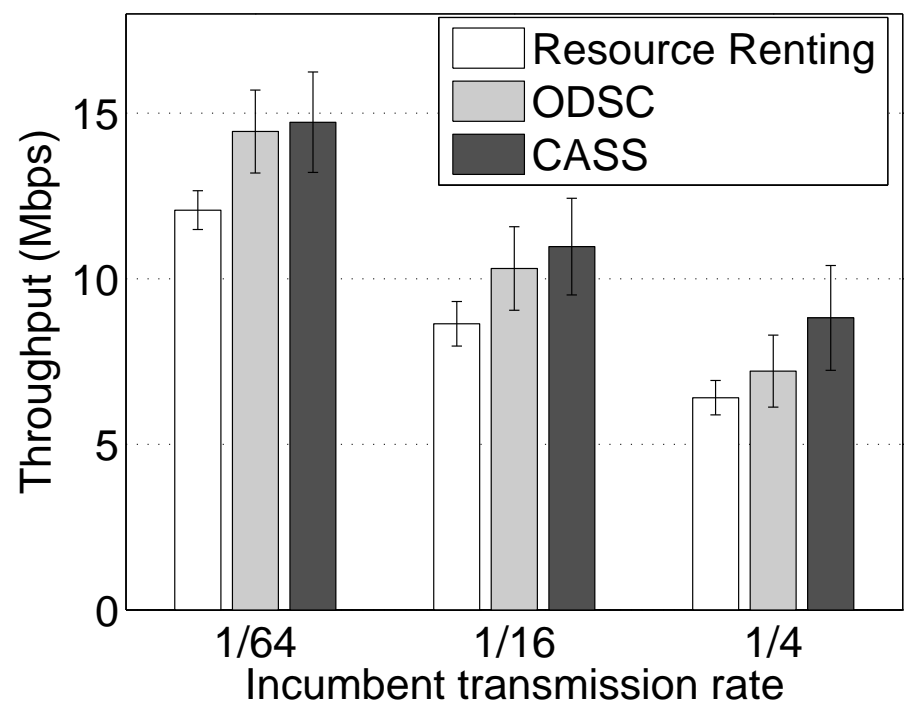

Figure 6.4: Throughput vs. Incumbent transmission rate in the 9-BS scenario.

Resource Renting and ODSC. In such a high self-interference environment, non-exclusive spectrum sharing schemes are inferior to exclusive spectrum sharing schemes. Figure 6.4 shows plots of average throughput per cell vs. incumbent transmission rate.

Figure 6.5 shows plots of the average throughput per cell vs. the channel switch delay. In the corresponding simulation experiments, we included four active incumbent transmitters that have the same transmission rate, viz 1/16. In inter-BS spectrum sharing, the channel switch delay is the biggest contributing factor to the control overhead. A recent study has found that the channel switch latency for most popular wireless cards is between $5 \mathrm{~ms}$ and $20 \mathrm{~ms}$ [43]. In Figure 6.5, we used the same range of values for the channel switch delay. As the channel switch delay increases, the control overhead caused by channel switch events during the channel contention procedures increases, thereby degrading the performance of ODSC and CASS. 


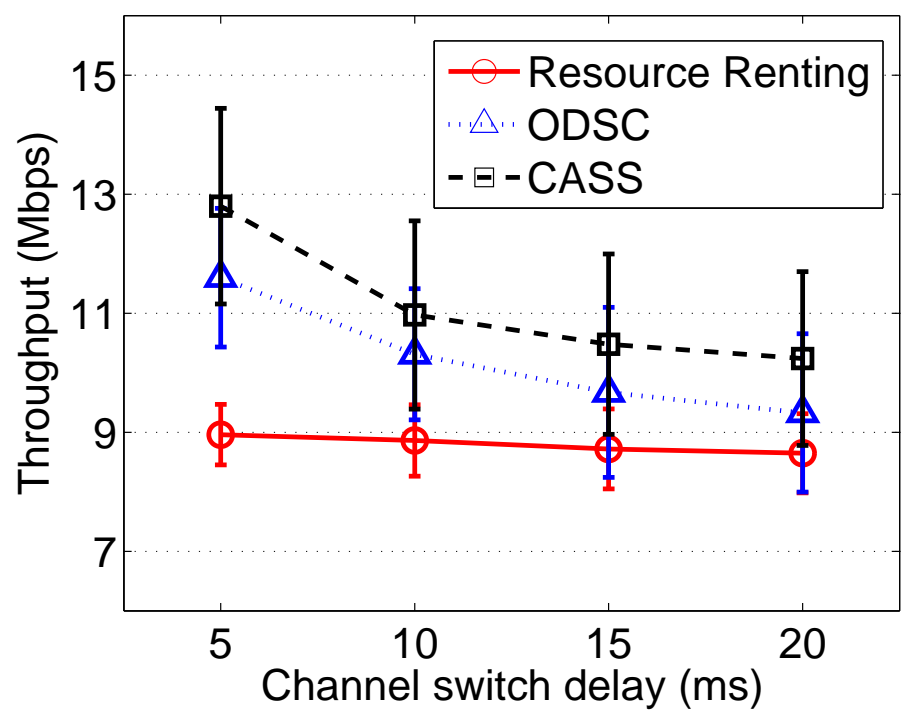

Figure 6.5: Throughput vs. Channel switch delay in the 9-BS scenario.

\subsection{Related Work}

In concept, 802.22's non-exclusive spectrum sharing mechanisms are somewhat similar to channel borrowing schemes proposed for cellular telephone networks [35]. In a channel borrowing scheme, a BS of one cell borrows channels from adjacent BSs. However, there is one important difference between 802.22's non-exclusive spectrum sharing and channel borrowing in cellular telephone networks — the former is a distributed process whereas the latter is a centralized process. 802.22 cells will be likely managed by different wireless service providers, and thus there will be no central entity that has the authority to manage channel assignments between 802.22 cells. For this reason, 802.22 carries out spectrum sharing (through channel borrowing) in a distributed manner. In contrast, channel assignment and sharing in cellular telephone systems are carried out in a centralized manner since cells in a given region are typically controlled by a centralized base station 
controller.

The research community's interest in the air interface for dynamic spectrum access has intensified in recent years. In [51], Sengupta et al. propose improvements for 802.22's air interface. Specifically, they formulated the 802.22 channel assignment problem as a vertex coloring problem, and proposed a coloring algorithm called Utility Graph Coloring (UGC) that is a centralized algorithm designed to maximize system utility (i.e., spectrum reuse). In [28], the authors studied techniques to maximize throughput in dynamic spectrum access networks under incumbent protection constraints.

\subsection{Summary}

The primary objective of 802.22 inter-BS spectrum sharing is to address the two major challenges in self-coexistence: minimize self-interference (among cells) and enable cells to acquire enough resources to satisfy the QoS of their admitted workloads (in opportunistic spectrum sharing environments). In this chapter, we identified the drawbacks of 802.22's spectrum sharing mechanisms and proposed a new inter-BS spectrum sharing protocol called Coexistence-Aware Spectrum Sharing (CASS). Our simulation results show that CASS outperforms 802.22's spectrum sharing mechanisms. The major contributions of this chapter include the following: (1) CASS gives us some level of insight on how to determine whether non-exclusive spectrum sharing or exclusive spectrum sharing should be employed; (2) CASS uses a channel selection strategy that incorporates spectrum sensing information to help satisfy incumbent coexistence requirements and improve network throughput; (3) CASS employs a channel contention procedure that was designed to avoid the drawbacks of ODSC's channel contention procedure. 


\section{Chapter 7}

\section{Conclusions and Future Work}

In this dissertation, we investigated three problems in the MAC protocol design for CR networks: (1) the rendezvous (control channel) establishment problem, (2) the channel assignment problem in an ad hoc CR network, and (3) the spectrum sharing problem between 802.22 WRANs. To address these problems, we provide solutions that comply with the MAC layer requirements of incumbent and self coexistence in CR networks.

\subsection{Conclusions}

The first set of research work in this dissertation presents a systematic approach, based on quorum systems, for designing and analyzing channel hopping $(\mathrm{CH})$ protocols that enable radio rendezvous in CR networks. We propose two synchronous designs of the $\mathrm{CH}$ system and two asynchronous $\mathrm{CH}$ systems for $\mathrm{CR}$ networks, all of which maximize the rendezvous diversity—-the number of pairwise rendezvous channels. Establishing rendezvous or control channels in multiple frequency channels implies that the secondary network is 
less vulnerable to the unpredictable appearance of incumbent signals. The second noteworthy feature of the proposed $\mathrm{CH}$ systems is that they guarantee that the time-to-rendezvous between channel hopping node pairs is upper bounded.

After the rendezvous is established, two communicating nodes in an ad hoc CR network have to negotiate and allocate channels to the link between them so as to carry out further data transmissions. In the OSS paradigm, the temporal and spatial spectrum variability caused by the incumbent users' spectrum utilization adds another dimension of complexity to the problem of channel assignment. Our work on segment-based channel assignment considers the problem when channel switching overhead is non-negligible. A novel channel assignment scheme is proposed, which assigns channels at the granularity of "segments" to minimize the channel switching overhead.

In infrastructure-based CR networks like 802.22 WRANs, there are two primary objectives for self-coexistence: minimize self-interference (among cells) and enable cells to acquire enough resources to satisfy the QoS of their admitted workloads (in OSS environments). In this dissertation, we identified the drawbacks of 802.22's spectrum sharing mechanisms and proposed a Coexistence-Aware Spectrum Sharing (CASS) that supported both non-exclusive spectrum sharing and exclusive spectrum sharing modes, and enhanced the existing 802.22 channel contention scheme.

\subsection{Future Work}

This research work mainly focuses on satisfying the incumbent and self coexistence requirements in the MAC protocol design for CR networks. The channel assignment problem is considered when channel switching overhead is significant. However, the link-based 
channel assignment strategy may outperform the segment-based approach in particular cases when channel switching overhead is small or negligible. Thus, part of the future work will lie in the development of a hybrid channel assignment approach for ad hoc CR networks, in which the channel assignment decisions can be made dynamically on a link or a segment basis so as to always offer the best trade-off in terms of incumbent coexistence and network performance under the OSS paradigm.

Beside the self/homogeneous coexistence, heterogeneous coexistence (which is a subproblem of horizontal coexistence as shown in Figure 1.1) has received more attention recently. The TV "white space" (TVWS) has the potential of providing significant bandwidth in frequencies that have very favorable propagation characteristics (i.e., long transmission ranges and capability of penetrating objects) [5]. Several standardization groups have initialized standardization efforts to enable the utilization of TVWS by leveraging CR technology, and these efforts include IEEE 802.22 Wireless Reginal Area Networks (WRAN) [31], IEEE 802.11af (WiFi over TVWS), Ecma 392 (WPAN over TVWS) [20], etc. All of these standards rely on CR technology to overcome the challenging interference issues between incumbent and secondary services as well as between secondary services.

There are two types of coexistence schemes: non-collaborative coexistence and collaborative coexistence schemes. A non-collaborative coexistence scheme is the only feasible approach when there are no means of coordination between the coexisting networks. In the existing literature, such an approach has been used to address the heterogeneous coexistence of WiFi and ZigBee networks $[29,72]$ as well as the homogeneous coexistence of uncoordinated WiFi deployments [43] and femto cell deployments $[59,68]$. A 
collaborative coexistence scheme can be employed when coexisting networks can coordinate their operations, and examples of such an approach include coexistence schemes for cellular networks $[25,48]$.

Existing coexistence schemes_-both non-collaborative and collaborative-cannot address the problems posed by the coexistence of heterogeneous CR networks. In TVWS, spectrum availability is expected to be limited due to the expected proliferation of TVWS unlicensed devise designs and high consumer adoption rate. The number of available channels will not be sufficient to provide a separate channel for each network, and thus some sort of a fine-grained mechanism for spectrum sharing or even channel sharing will be needed to minimize adjacent and co-channel interference. Non-collaborative schemes are relatively simple and they cannot achieve fine-grained spectrum sharing nor channel sharing due to the lack of coordination between the coexisting networks. Collaborative schemes enable fine-grained spectrum sharing or channel sharing but are limited to only homogeneous coexistence. Devising a collaborative scheme for heterogeneous coexistence is very challenging for a number of reasons. First, coexisting networks would need to exchange spectrum sharing negotiation information and other control information over a common control channel. The realization of such a channel may require a broad standardization effort across secondary systems and would be costly. Second, even if an effective means of inter-network communications between coexisting networks exists, implementation of collaborative strategies would only be possible with time synchronization across all devices from different networks. Achieving synchronization over a potentially large number of coexisting TVWS networks may not be feasible.

Therefore, part of the future research will commit to improving the heterogeneous 
coexistence of CR networks over the TVWS. 


\section{Bibliography}

[1] I. F. Akyildiz, W. Y. Lee, M. C. Vuran, and S. Mohanty. NeXt Generation/Dynamic Spectrum Access/Cognitive Radio Wireless Networks: A Survey. Computer Networks Journal (Elsevier), 50(13):2127-2159, September 2006.

[2] M. Alicherry, R. Bhatia, and L. E. Li. Joint Channel Assignment and Routing for Throughput Optimization in Multi-Radio Wireless Mesh Networks. In Proc. of ACM MobiCom, pages 58-72, September 2005.

[3] E. Aryafar, Omer Gurewitz and Edward W. Knightly. Distance-1 Constrained Channel Assignment in Single Radio Wireless Mesh Networks. In Proc. of IEEE INFOCOM, pages 762-770, April 2008.

[4] P. Bahl, R. Chandra and J. Dunagan. SSCH: Slotted Seeded Channel Hopping for Capacity Improvement in IEEE 802.11 Ad Hoc Wireless Networks. In Proc. of ACM MobiCom, pages 216-230, September 2004.

[5] P. Bahl, R. Chandra, T. Moscibroda, R. Murty, and M. Welsh. White Space Networking with Wi-Fi like Connectivity. In Proc. of Sigcomm, pages 27-38, August 2009.

[6] K. Bian and J. Park. Asynchronous Channel Hopping for Establishing Rendezvous in Cognitive Radio Networks. In Proc. of IEEE INFOCOM, Mini-conference, April 2011.

[7] K. Bian, J. Park, and R. Chen. A Quorum-based Framework for Establishing Control Channels in Dynamic Spectrum Access Networks. In Proc. of ACM MobiCom, pages 25-36, September 2009.

[8] S. Brahma and M. Chatterjee. Mitigating Self-Interference Among IEEE 802.22 Networks: A Game Theoretic Perspective. In Proc. of IEEE Globecom, pages 1-6, November 2009.

[9] V. Brik, E. Rozner, S. Banarjee, and P. Bahl. DSAP: A Protocol for Coordinated Spectrum Access. In Proc. of IEEE DySPAN, pages 611-614, November 2005. 
[10] D. Cabric, S. M. Mishra, and R. W. Brodersen. Implementation Issues in Spectrum Sensing for Cognitive Radios. In Proc. of the 38th Asilomar Conference on Signals, Systems, and Computers, November 2004.

[11] L. Cao and H. Zheng. DSAP: Distributed Spectrum Allocation via Local Bargaining. In Proc. of IEEE Second Annual IEEE Communications Society Conference on Sensor and Ad Hoc Communications and Networks, (Secon), pages 475-486, September 2005.

[12] R. Chen, J. Park, and K. Bian. Robust Distributed Spectrum Sensing in Cognitive Radio Networks. In Proc. of IEEE Infocom Mini-conference, April 2008.

[13] R. Chen, J. Park, and J. H. Reed. Defense against Primary User Emulation Attacks in Cognitive Radio Networks. IEEE Journal on Selected Areas in Communcations Special Issue on Cognitive Radio Theory and Applications, 26(1):25-37, January 2008.

[14] R. Chen, J. Park, Y. T. Hou, and J. H. Reed. Toward Secure Distributed Spectrum Sensing in Cognitive Radio Networks. IEEE Communications Magazine Special Issue on Cognitive Radio Communications, 46(4):50-55, April 2008.

[15] T. Chen, H. Zhang, G. M. Maggio, and I. Chlamtac. CogMesh: A Cluster-based Cognitive Radio Network. In Proc. of IEEE DySpan, pages 168-178, April 2007.

[16] C. J. Colbourn and E. J. H. Dinitz. The CRC Handbook of Combinatorial Designs. CRC Press, 1996.

[17] C. Cordeiro and K. Challapali. C-MAC: A Cognitive MAC Protocol for MultiChannel Wireless Networks. In Proc. of IEEE DySpan, pages 147-157, April 2007.

[18] C. M. Cordeiro, K. Challapali, D. Birru, and N. S. Shankar. IEEE 802.22: The First Worldwide Wireless Standard Based on Cognitive Radios. IEEE DySPAN, November 2005.

[19] L.A. DaSilva and I. Guerreiro. Sequence-Based Rendezvous for Dynamic Spectrum Access. In Proc. of IEEE DySpan, pages 1-7, October 2008.

[20] Ecma International. ECMA-392: MAC and PHY for Operation in TV White Space. 1st Edition, December 2009.

[21] Federal Communications Commission. Facilitating Opportunities for Flexible, Efficient, and Reliable Spectrum Use Employing Spectrum Agile Radio Technologies. ET Docket No. 03-108, December 2003. 
[22] Federal Communications Commission. Unlicensed Operation in the TV Broadcast Bands and Additional Spectrum for Unlicensed Devices below $900 \mathrm{MHz}$ in the $3 \mathrm{GHz}$ Band ET Docket No. 04-186, May 2004.

[23] F. Fitzek, D. Angelini, G. Mazzini, and M. Zorzi. Design and Performance of An Enhanced IEEE 802.11 MAC Protocol for Multihop Coverage Extension. IEEE Wireless Communications, 10(6):30-39, December 2003.

[24] G. Ganesan and Y. Li. Cooperative Spectrum Sensing in Cognitive Radio Networks. In Proc. of IEEE DySPAN, pages 137-143, November 2005.

[25] M. Ghaderi, A. Sridharan, H. Zang, D. Towsley, and R. Cruz. TCP-Aware Resource Allocation in CDMA Networks. In Proc. of ACM MobiCom, pages 215-226, September 2006.

[26] D. Grandblaise and W. Hu. Inter Base Stations Adaptive On Demand Channel Contention for IEEE 802.22 WRAN Self Coexistence. IEEE docs: IEEE 802.2207/0024r0, Jannuary 2007.

[27] S. Haykin. Cognitive Radio: Brain-empowered Wireless Communications. IEEE Journal on Selected Areas in Communications Special Issue on Cognitive Radio Theory and Applications, 23(2):201-220, February 2005.

[28] S. Huang, X. Liu, and Z. Ding. Opportunistic Spectrum Access in Cognitive Radio Networks. In Proc. of IEEE INFOCOM, pages 1427-1435, April 2008.

[29] J. Huang, G. Xing, G. Zhou, and R. Zhou. Beyond Co-existence: Exploiting WiFi White Space for ZigBee Performance Assurance. In Proc. of IEEE ICNP, October 2010.

[30] IEEE 802.19 Task Group 1. Wireless Coexistence in the TV White Space. http: //www. ieee802.org/19/pub/TG1.html.

[31] IEEE 802.22 Working Group. www.ieee802.org.

[32] IEEE 802.22 Working Group. Reviews of Channel Model. IEEE docs: 22-05-007000-0000, August 2005.

[33] IEEE 802.22 Working Group. ETRI FT Philips Samsung Proposal. IEEE docs: 22-06-0005-01-0000, Jan. 2006.

[34] J. Jia, Q. Zhang, and X. S. Shen. HC-MAC: A Hardware-constrained Cognitive MAC for Efficient Spectrum Management. IEEE Journal on Selected Areas in Communications Special Issue on Cognitive Radio Theory and Applications, 26(1):106-117, January 2008. 
[35] H. Jiang, and S. S. Rappaport. CBWL: A New Channel Assignment and Sharing Method for Cellular Communications Systems. IEEE Transactions on Vehicular Technology, 43(2):313-321, May 1994,.

[36] J. R. Jiang, Y. C. Tseng and T. Lai. Quorum-based Asynchronous Power-saving Protocols for IEEE 802.11 Ad Hoc Networks. ACM Journal on Mobile Networks and Applications, 10(1-2):169-181, February 2005.

[37] D. B. Johnson, D. A. Maltz, and Y.-C. Hu. The Dynamic Source Routing Protocol for Mobile Ad Hoc Networks (DSR) (Internet-Draft). Mobile Ad-hoc Network (MANET) Working Group, IETF, July 2004.

[38] H. Kim and K. G. Shin. Efficient Discovery of Spectrum Opportunities with MACLayer Sensing in Cognitive Radio Networks. IEEE Trans. on Mobile Computing, 7(5):533-545, Jannuary 2008.

[39] P. Kyasanur and N. Vaidya. Capacity of Multi-channel Wireless Networks: Impact of Number of Channels and Interfaces. In Proc. of ACM MobiCom, pages 43-57, September 2005.

[40] L. Lazos, S. Liu, and M. Krunz. Mitigating Control-Channel Jamming Attacks in Multi-channel Ad Hoc Networks. In Proc. of ACM WiSec, pages 169-180, March 2009.

[41] C. Ko and H. Wei. Game Theoretical Resource Allocation for Inter-BS Coexistence in IEEE 802.22. IEEE Transactions on Vehicular Technology, 59(4):1729-1744, May 2010.

[42] W.-S. Luk and T.-T. Wong. Two New Quorum Based Algorithms for Distributed Mutual Exclusion. In Proc. of IEEE ICDCS, pages 100-106, May 1997.

[43] A. Mishra, V. Shrivastava, D. Agrawal, S. Banerjee, and S. Ganguly. Distributed Channel Management in Uncoordinated Wireless Environments. In Proc. of ACM Mobicom, pages 170-181, September 2006.

[44] J. Mo, H.-S.W. So, and J. Walrand. Comparison of Multichannel MAC Protocols. IEEE Transactions on Mobile Computing, 7(1):50-65, January 2008.

[45] H. Nan, T.-I. Hyon, and S.-J. Yoo. Distributed Coordinated Spectrum Sharing MAC Protocol for Cognitive Radio In Proc. of IEEE DySpan, pages 240-249, April 2007.

[46] M. Naor and A. Wool. The Load, Capacity, and Availability of Quorum Systems. SIAM Journal on Computing, 27(2):214-225, 1998. 
[47] C. Perkins and E. Royer. Ad Hoc On-Demand Distance Vector Routing. In Proc. of the 2nd IEEE Workshop on Mobile Computing Systems and Applications, pages 90-100, February 1999.

[48] F. Qian, Z. Wang, A. Gerber, Z.M. Mao, S. Sen, and O. Spatscheck. TOP: Tail Optimization Protocol for Cellular Radio Resource Allocation. In Proc. of IEEE ICNP, October 2010.

[49] S. Ramanathan. A Unified Framework and Algorithm for (T/F/C)DMA Channel Assignment in Wireless Networks. In Proc. of IEEE INFOCOM, pages 900-907, April 1997.

[50] T. S. Rappaport. Wireless Communications: Principles and Practice. Upper Saddle River, NJ: Prentice Hall, 2001.

[51] S. Sengupta, S. Brahma, M. Chatterjee and S. Shankar. Enhancements to Cognitive Radio Based IEEE 802.22 Air-Interface. IEEE ICC, pages 5155-5160, June 2007.

[52] S. Shankar N, C. Cordeiro, and K. Challapali. Spectrum agile radios: utilization and sensing architectures. In Proc. of IEEE DySPAN, pages 160-169, November 2005.

[53] S. J. Shellhammer. Estimation of Packet Error Rate Caused by Interference using Analytic Techniques-A Coexistence Assurance Methodology. IEEE docs: P802.1905/0028r1, September 2005.

[54] M. D. Silvius, F. Ge, A. Young, A. B. MacKenzie, and C. W. Bostian. Smart Radio: Spectrum Access for First Responders. In Proc. of SPIE, Vol. 6980, Wireless Sensing and Processing III, April 2008.

[55] J. So and N. Vaidya. Multi-Channel MAC for Ad Hoc Networks: Handling MultiChannel Hidden Terminals Using a Single Transceiver. In Proc. of ACM MobiHoc, pages 222-233, May 2004.

[56] H. W. So, J. Walrand and J. Mo. McMAC: A Multi-Channel MAC Proposal for Ad Hoc Wireless Networks. In Proc. of IEEE WCNC, pages 334-339, March 2007.

[57] D. R. Stinson. Combinatorial Designs: Constructions and Analysis. Springer-Verlag, 2003.

[58] M. Strasser, C. Popper, S. Capkun, and M. Cagalj. Jamming-resistant Key Establishment using Uncoordinated Frequency Hopping. In Proc. of IEEE Symposium on Security and Privacy, pages 64-78, May 2008. 
[59] K. Sundaresan and S. Rangarajan. Efficient Resource Management in OFDMA Femto Cells. In Proc. of ACM MobiHoc, pages 33-42, May 2009.

[60] N. Theis, R. Thomas and L. DaSilva. Rendezvous for Cognitive Radios. IEEE Transactions on Mobile Computing, 10(2):216-227, February 2011.

[61] Y.-C. Tseng, C.-S. Tsu, and T.-Y. Hsieh. Power-saving Protocols for IEEE 802.11based Multi-hope Ad Hoc Networks. In Proc. of IEEE INFOCOM, pages 200-209, June 2002.

[62] A. Tzamaloukas and J. J. Garcia-Luna-Aceves. Channel-Hopping Multiple Access. In Proc. of IEEE ICC, pages 415-419, June 2000.

[63] The Network Simulator (ns-2). www.isi.edu/nsnam/ns/.

[64] R. Vedantham, S. Kakumanu, S. Lakshmanan, and R. Sivakumar. Component-based Channel Assignment in Single Radio, Multi-channel Ad Hoc Networks. In Proc. of ACM MobiCom, pages 378-389, September 2006.

[65] D. B. West. Introduction to Graph Theory. 2nd ed., Prentice Hall, 2001.

[66] B. Wild, and K. Ramchandran. Detecting primary receivers for cognitive radio applications. In Proc. of IEEE DySPAN, pages 124-130, November 2005.

[67] S.-L. Wu, C.-Y. Lin, Y.-C. Tseng and J.-L. Sheu. A New Multi-channel MAC Protocol with On-demand Channel Assignment for Multi-hop Mobile Ad Hoc Networks. In Proc. of International Symposium on Parallel Architectures, Algorithms and Networks, I-SPAN, December 2000.

[68] J. Yun and K. Shin. CTRL: A Self-organizing Femtocell Management Architecture for Co-channel Deployment. In Proc. of ACM MobiCom, pages 61-72, September 2010.

[69] J. Zhao, H. Zheng, and G.-H. Yang. Distributed Coordination in Dynamic Spectrum Allocation Networks. In Proc. of IEEE DySpan, pages 259-268, November 2005.

[70] H. Zheng and L. Cao. Device-centric Spectrum Management. In Proc. of IEEE DySpan, pages 56-65, November 2005.

[71] R. Zheng, J. C. Hou, and L. Sha. Asynchronous Wakeup for Ad Hoc Networks. In Proc. of ACM ACM MobiHoc, pages 35-45, June 2003.

[72] R. Zhou, Y. Xiong, G. Xing, L. Sun, and J. Ma. ZiFi: Wireless LAN Discovery via ZigBee Interference Signatures. In Proc. of ACM Mobicom, pages 49-60, September 2010. 


\section{Appendix A}

\section{Authors' Publications}

\section{Book Chapters}

1. J. Park, and K. Bian, "Security of Cognitive Radios," in Encyclopedia of Cryptography and Security (2nd Ed.), Henk C.A. van Tilborg, and Sushil Jajodia (Eds.), Springer, 2011.

2. J. Park, K. Bian, and R. Chen, "Cognitive Radio Network Security," in Cognitive Radio Communications and Networks: Principles and Practice, A. Wyglinski, M. Nekovee, and T. Hou (Eds.), Academic Press/Elsevier, 2010. ISBN: 978-0-12374715-0.

\section{Journal Papers}

1. K. Bian, J. Park, and R. Chen, Control Channel Establishment in Cognitive Radio Networks using Channel Hopping, IEEE Journal on Selected Areas in Communications (IEEE JSAC), Vol. 29(4):689-703, April 2011. 
2. R. Chen, J. Park, and K. Bian, Robustness against Byzantine Failures in Distributed Spectrum Sensing, under review.

\section{Conference Papers}

1. K. Bian and J. Park, Asynchronous Channel Hopping for Establishing Rendezvous in Cognitive Radio Networks, IEEE INFOCOM 2011, Mini-conference, April 2011.

2. K. Bian and J. Park, Addressing the Hidden Incumbent Problem in 802.22 Networks, the Software Defined Radio (SDR) Forum 2009 Technical Conference (SDR 2009), December 2009.

3. K. Bian, J. Park, and R. Chen, A Quorum-based Framework for Establishing Control Channels in Dynamic Spectrum Access Networks, the 15th ACM Annual International Conference on Mobile Computing and Networking (ACM MobiCom 2009), September 2009.

4. K. Bian and J. Park, A Coexistence-Aware Spectrum Sharing Protocol for 802.22 WRANs, the 18th IEEE International Conference on Computer Communications and Networks (IEEE ICCCN 2009), August 2009.

5. K. Bian, J. Park, M. Hsiao, F. Belanger, and J. Hiller, Evaluation of Online Resources in Assisting Phishing Detection, the 9th IEEE/IPSJ International Symposium on Applications and the Internet (IEEE SAINT 2009), July 2009.

6. R. Chen, J. Park and K. Bian, Robust Distributed Spectrum Sensing in Cognitive Radio Networks, IEEE INFOCOM 2008, Mini-conference, April 2008. 
7. K. Bian and J. Park, Security Vulnerabilities in IEEE 802.22, the Fourth ICST International Wireless Internet Conference (ICST WICON 2008), November 2008.

8. K. Channakeshava, K. Bian, M. Hsiao, J. Park, R. Crossler, P. Aggarwal, F. Belanger, and J. Hiller, On Providing Automatic Parental Consent over Information Collection from Children, the 2008 International Conference on Security and Management (SAM 2008), August 2008.

9. K. Bian and J. Park, Segment-Based Channel Assignment in Cognitive Radio Ad Hoc Networks, the Second ICST International Conference on Cognitive Radio Oriented Wireless Networks and Communications (ICST CrownCom 2007), August 2007.

10. R. Crossler, F. Belanger, J. Hiller, P. Aggarwal, K. Channakeshava, K. Bian, J. Park, and M. Hsiao, Parents and the Internet: Privacy Awareness, Practices, and Control, in the 14th Americas Conference on Information Systems (AMCIS 2007), August 2007.

11. K. Bian, J. Park and R. Chen, Stasis Trap: Cross-Layer Stealthy Attacks in Wireless Ad Hoc Networks, the 49th IEEE Global Telecommunications Conference (IEEE Globecom 2006), November 2006.

12. K. Bian and J. Park, MAC-Layer Misbehaviors in Multi-Hop Cognitive Radio Networks, the 2006 US-Korea Conference on Science, Technology, and Entrepreneurship (UKC 2006), August 2006. 